\title{
A lattice point problem associated with two polynomials
}

\author{
by \\ Manfred Peter (Freiburg)
}

1. Introduction. B. Lichtin [5] proves the following result: Let $g_{\nu} \in$ $\mathbb{R}[x, y], \nu=1,2$, be two polynomials which are nondegenerate with respect to their polygon at infinity and hypoelliptic on $[1, \infty)^{2}$. Define

$$
R\left(A_{1}, A_{2}\right):=\#\left\{(x, y) \in \mathbb{N}^{2} \mid g_{\nu}(x, y) \leq A_{\nu}, \nu=1,2\right\}
$$

for $A_{1}, A_{2}>0$. There are finitely many sets

$$
\mathcal{R}_{j}=\left\{\left(A_{1}, A_{2}\right) \in[1, \infty)^{2} \mid A_{1}^{b_{j}} \leq A_{2} \leq A_{1}^{B_{j}}\right\}
$$

with $0 \leq b_{j}<B_{j} \leq \infty$ and $[1, \infty)^{2}=\bigcup_{j} \mathcal{R}_{j}$, polynomials $p_{j}(u, v) \in \mathbb{R}[u, v]$ which are positive outside some compact subset of $[1, \infty)^{2}$ and constants $u_{j}, v_{j}>0$ so that the following asymptotics holds: If $\mathcal{R}_{\infty} \subseteq \mathcal{R}_{j}$ is an unbounded connected semialgebraic set with $\operatorname{dist}\left(\left(A_{1}, A_{2}\right), \partial \mathcal{R}_{j}\right) \rightarrow \infty$ as $\left(A_{1}, A_{2}\right) \rightarrow(\infty, \infty)$ in $\mathcal{R}_{\infty}$ then with some $\Theta>0$,

$$
R\left(A_{1}, A_{2}\right)=A_{1}^{u_{j}} A_{2}^{v_{j}} p_{j}\left(\log A_{1}, \log A_{2}\right)+O\left(A_{1}^{u_{j}-\Theta} A_{2}^{v_{j}-\Theta}\right)
$$

as $\left(A_{1}, A_{2}\right) \rightarrow(\infty, \infty)$ in $\mathcal{R}_{\infty}$.

Lichtin gives an explicit description of $\mathcal{R}_{j}, u_{j}, v_{j}$ in terms of the region of analyticity of some Dirichlet series which is associated with the polynomials $g_{\nu}$. It is the aim of this paper to derive a much sharper asymptotic expansion of $R\left(A_{1}, A_{2}\right)$ under conditions on the polynomials $g_{\nu}$ which are in some sense complementary to those of Lichtin.

Let

$$
g_{\nu}(x, y)=\sum_{i+j \leq d_{\nu}} a_{i j}^{(\nu)} x^{i} y^{j} \in \mathbb{Z}[x, y], \quad \nu=1,2,
$$

be polynomials with nonnegative integer coefficients and $a_{d_{\nu} 0}^{(\nu)} a_{0 d_{\nu}}^{(\nu)} \neq 0$. Define

$$
\widetilde{g}_{\nu}(x, y)=\sum_{i+j=d_{\nu}} a_{i j}^{(\nu)} x^{i} y^{j} \in \mathbb{Z}[x, y]
$$

2000 Mathematics Subject Classification: Primary 11P21.

Key words and phrases: lattice points, polynomials. 
and let the functions $\widetilde{y}_{\nu}:\left[0, \widetilde{\xi}_{\nu}\right] \rightarrow \mathbb{R}_{0}^{+}$and $\widetilde{x}_{\nu}:\left[0, \widetilde{\eta}_{\nu}\right] \rightarrow \mathbb{R}_{0}^{+}$be implicitly defined by

$$
\begin{array}{ll}
\widetilde{g}_{\nu}\left(x, \widetilde{y}_{\nu}(x)\right)=1 & \text { for } 0 \leq x \leq \widetilde{\xi}_{\nu}, \widetilde{y}_{\nu}\left(\widetilde{\xi}_{\nu}\right)=0 \\
\widetilde{g}_{\nu}\left(\widetilde{x}_{\nu}(y), y\right)=1 & \text { for } 0 \leq y \leq \widetilde{\eta}_{\nu}, \widetilde{x}_{\nu}\left(\widetilde{\eta}_{\nu}\right)=0
\end{array}
$$

Assume that the rational function

$$
\widetilde{g}_{1}(1, y)^{d_{2}} / \widetilde{g}_{2}(1, y)^{d_{1}}
$$

is not constant. Assume further that $\widetilde{g}_{\nu}$ is not of the form

$$
a_{0 d_{\nu}}^{(\nu)}(y-b x)^{d_{\nu}} \quad \text { or } \quad a_{d_{\nu} 0}^{(\nu)}(x-c y)^{d_{\nu}}
$$

with some $b, c \in \mathbb{R}$. This paper is devoted to the proof of

TheOREM 1.1. There is a decomposition $0=C_{-1}^{*}<C_{0}^{*}<\ldots<C_{m}^{*}<$ $C_{m+1}^{*}=\infty$ and constants $K, \varepsilon>0$ with the property: For $0 \leq \mu \leq$ $m+1$ and $A_{1}, A_{2} \geq K$ with $C:=A_{2} A_{1}^{-d_{2} / d_{1}} \in\left(C_{\mu-1}^{*}+\min \left\{A_{1}, A_{2}\right\}^{-\varepsilon}\right.$, $\left.C_{\mu}^{*}-\min \left\{A_{1}, A_{2}\right\}^{-\varepsilon}\right)$ we have

$$
\begin{aligned}
R\left(A_{1}, A_{2}\right)= & \operatorname{area}\left(\left\{(x, y) \in \mathbb{R}^{2} \mid x, y \geq 0, g_{\nu}(x, y) \leq A_{\nu}, \nu=1,2\right\}\right) \\
& +T_{1}^{(\mu)}\left(A_{1}\right)+T_{2}^{(\mu)}\left(A_{2}\right)+U_{1}^{(\mu)}\left(A_{1}\right)+U_{2}^{(\mu)}\left(A_{2}\right) \\
& -\frac{1}{2} \min \left\{A_{1}^{1 / d_{1}} \widetilde{\xi}_{1}, A_{2}^{1 / d_{2}} \widetilde{\xi}_{2}\right\}-\frac{1}{2} \min \left\{A_{1}^{1 / d_{1}} \widetilde{\eta}_{1}, A_{2}^{1 / d_{2}} \widetilde{\eta}_{2}\right\} \\
& +O\left(A_{1}^{46 /\left(73 d_{1}\right)}\left(\log A_{1}\right)^{315 / 146}\right) \\
& +O\left(A_{2}^{46 /\left(73 d_{2}\right)}\left(\log A_{2}\right)^{315 / 146}\right) .
\end{aligned}
$$

The representation

$$
\begin{aligned}
T_{\nu}^{(\mu)}\left(A_{\nu}\right)= & A_{\nu}^{\left(1 / d_{\nu}\right)\left(1-1 /\left(p_{\mu, \nu}+2\right)\right)} \sum_{l=1}^{L_{\nu}^{(\mu)}} H_{\nu l}^{(\mu)}\left(A_{\nu}^{1 / d_{\nu}}\right) \\
& +O\left(A_{\nu}^{\left(1 / d_{\nu}\right)\left(1-1 /\left(p_{\mu, \nu}+2\right)-1 /\left(p_{\mu, \nu}+2\right)^{2}\right)} \log A_{\nu}\right)
\end{aligned}
$$

holds with $p_{\mu, \nu} \in \mathbb{N}$ and $H_{\nu l}^{(\mu)}$ periodic functions which are given by absolutely convergent Fourier series. Furthermore, with some $q_{\mu, \nu} \in \mathbb{N}$,

$$
U_{\nu}^{(\mu)}\left(A_{\nu}\right)=O_{\delta}\left(A_{\nu}^{\left(1 / d_{\nu}\right)\left(1-1 / q_{\mu, \nu}+\delta\right)}\right)
$$

for each $\delta>0 . T_{\nu}^{(\mu)}$ (resp. $\left.U_{\nu}^{(\mu)}\right)$ can only appear if there is a zero of $\widetilde{y}_{\nu}^{\prime \prime}$ or $\widetilde{x}_{\nu}^{\prime \prime}$ at which $\widetilde{y}_{\nu}^{\prime}$ or $\widetilde{x}_{\nu}^{\prime}$ is rational (resp. irrational). $p_{\mu, \nu}\left(\right.$ resp. $\left.q_{\mu, \nu}\right)$ is at most $d_{\nu}-2$.

The proof consists of three parts. In a combinatorial part the set in which the lattice points are counted is dissected into finitely many subsets. Each of these has a boundary which is described by one single algebraic curve. These local problems can be treated in just the same way as in [9]. That is the analytical part which uses exponential sum estimates. The last section is 
concerned with the question whether the requirements of this local analysis can be met by imposing suitable conditions on the relative position of $A_{1}$ and $A_{2}$. This part is again combinatorial in nature.

In principle it is possible to give an explicit description of $C_{\mu}^{*}, T_{\nu}^{(\mu)}$, $U_{\nu}^{(\mu)}$ and $\varepsilon$ in terms of $\widetilde{y}_{\nu}, \widetilde{x}_{\nu}$ and the coefficients of the $g_{\nu}$. In Sections 4 , 5 and 6 this is done for the local lattice point asymptotics. In the global asymptotics an explicit description of all the constants and functions would make it necessary to distinguish between a lot of cases. Therefore I chose the above formulation which lays emphasis on the structure of the asymptotic formula and not on explicit calculations.

In the following $\varepsilon>0$ will be a sufficiently small constant, $K>0$ a sufficiently large constant and $K_{j}>0, j \in \mathbb{N}$, constants depending only on the $g_{\nu}$. Furthermore $\psi(x):=x-[x]-1 / 2$.

I would like to thank the referee for a suggestion which led to an improvement of the paper.

2. Reduction to algebraic boundary curves. Define an auxiliary function $h:\left[0, \widetilde{\xi}_{1}\right] \rightarrow \mathbb{R}^{+}$by $h(x):=\widetilde{g}_{2}\left(x, \widetilde{y}_{1}(x)\right)$.

LEMMA 2.1. The function $h$ is not constant.

Proof. Assume $h(x)=\lambda \in \mathbb{R}^{+}$for each $x \in\left[0, \widetilde{\xi}_{1}\right]$. Then for $0<x<\widetilde{\xi}_{1}$,

$$
\begin{aligned}
\lambda^{d_{1}} \widetilde{g}_{1}\left(1, x^{-1} \widetilde{y}_{1}(x)\right)^{d_{2}} & =\lambda^{d_{1}} x^{-d_{1} d_{2}} \widetilde{g}_{1}\left(x, \widetilde{y}_{1}(x)\right)^{d_{2}}=h(x)^{d_{1}} x^{-d_{1} d_{2}} \\
& =x^{-d_{1} d_{2}} \widetilde{g}_{2}\left(x, \widetilde{y}_{1}(x)\right)^{d_{1}}=\widetilde{g}_{2}\left(1, x^{-1} \widetilde{y}_{1}(x)\right)^{d_{1}} .
\end{aligned}
$$

With $\widetilde{y}_{1}(x) \rightarrow \widetilde{y}_{1}(0)>0$ as $x \rightarrow 0+0$ it follows that $x^{-1} \widetilde{y}_{1}(x) \rightarrow \infty$ as $x \rightarrow 0+0$. Therefore the identity $\lambda^{d_{1}} \widetilde{g}_{1}(1, y)^{d_{2}}=\widetilde{g}_{2}(1, y)^{d_{1}}$ holds for infinitely many $y$ and consequently it holds as a polynomial identity. This contradicts the assumption on (1.1).

Define the homogeneous polynomials of degree $d_{\nu}$

$$
g_{\nu}(\tau, x, y):=\sum_{i+j \leq d_{\nu}} a_{i j}^{(\nu)} \tau^{d_{\nu}-i-j} x^{i} y^{j} \in \mathbb{Z}[\tau, x, y]
$$

Define

$$
\begin{aligned}
& j_{0}^{(\nu)}:=\min \left\{1 \leq j \leq d_{\nu} \mid a_{d_{\nu}-j, j}^{(\nu)} \neq 0\right\} \\
& i_{0}^{(\nu)}:=\min \left\{1 \leq i \leq d_{\nu} \mid a_{i, d_{\nu}-i}^{(\nu)} \neq 0\right\} .
\end{aligned}
$$

For $0 \leq \tau<\left(a_{00}^{(\nu)}\right)^{-1 / d_{\nu}}$ the functions $y_{\nu}(\tau, \cdot):\left[0, \xi_{\nu}(\tau)\right] \rightarrow \mathbb{R}_{0}^{+}$and $x_{\nu}(\tau, \cdot):$ $\left[0, \eta_{\nu}(\tau)\right] \rightarrow \mathbb{R}_{0}^{+}$are implicitly defined by

$$
\begin{array}{ll}
g_{\nu}\left(\tau, x, y_{\nu}(\tau, x)\right)=1, & 0 \leq x \leq \xi_{\nu}(\tau), y_{\nu}\left(\tau, \xi_{\nu}(\tau)\right)=0 \\
g_{\nu}\left(\tau, x_{\nu}(\tau, y), y\right)=1, & 0 \leq y \leq \eta_{\nu}(\tau), x_{\nu}\left(\tau, \eta_{\nu}(\tau)\right)=0
\end{array}
$$


Both are strictly decreasing and inverse to each other. For $0 \leq x<\xi_{\nu}(\tau)$,

$$
\begin{aligned}
& g_{\nu y}\left(\tau, x, y_{\nu}(\tau, x)\right) \\
\geq & \max \left\{a_{d_{\nu}-j_{0}^{(\nu)}, j_{0}^{(\nu)}}^{(\nu)} j_{0}^{(\nu)} x^{d_{\nu}-j_{0}^{(\nu)}} y_{\nu}(\tau, x)^{j_{0}^{(\nu)}-1}, a_{0 d_{\nu}}^{(\nu)} d_{\nu} y_{\nu}(\tau, x)^{d_{\nu}-1}\right\}>0 .
\end{aligned}
$$

Consequently, $y_{\nu}$ is $C^{\infty}$ on an open neighbourhood of

$$
\left\{(\tau, x) \mid 0 \leq \tau<\left(a_{00}^{(\nu)}\right)^{-1 / d_{\nu}}, 0 \leq x<\xi_{\nu}(\tau)\right\}
$$

by the implicit function theorem. If $j_{0}^{(\nu)}=1$ then $(2.1)$ is also valid for $x=\xi_{\nu}(\tau)$ and consequently $y_{\nu}$ is $C^{\infty}$ on an open neighbourhood of

$$
\left\{(\tau, x) \mid 0 \leq \tau<\left(a_{00}^{(\nu)}\right)^{-1 / d_{\nu}}, 0 \leq x \leq \xi_{\nu}(\tau)\right\}
$$

We have $\eta_{\nu}(\tau)=y_{\nu}(\tau, 0)$ for $0 \leq \tau<\left(a_{00}^{(\nu)}\right)^{-1 / d_{\nu}}$. Consequently, $\eta_{\nu}$ is $C^{\infty}$ in this interval and $\eta_{\nu}(\tau)=\widetilde{\eta}_{\nu}+O(\tau)$ as $\tau \rightarrow 0$. Analogous results are valid for $x_{\nu}$.

For $A_{1}, A_{2}>\max \left\{a_{00}^{(1)}, a_{00}^{(2)}, 1\right\}$ define $f_{A_{1}, A_{2}}:\left[0, \varrho_{A_{1}, A_{2}}\right] \rightarrow \mathbb{R}_{0}^{+}$by

$$
\begin{aligned}
f_{A_{1}, A_{2}}(x) & :=\min \left\{A_{1}^{1 / d_{1}} y_{1}\left(A_{1}^{-1 / d_{1}}, A_{1}^{-1 / d_{1}} x\right), A_{2}^{1 / d_{2}} y_{2}\left(A_{2}^{-1 / d_{2}}, A_{2}^{-1 / d_{2}} x\right)\right\}, \\
\varrho_{A_{1}, A_{2}} & :=\min \left\{A_{1}^{1 / d_{1}} \xi_{1}\left(A_{1}^{-1 / d_{1}}\right), A_{2}^{1 / d_{2}} \xi_{2}\left(A_{2}^{-1 / d_{2}}\right)\right\} .
\end{aligned}
$$

Then the following equivalence holds for $x, y \geq 0$ :

$$
\begin{aligned}
& g_{\nu}(x, y) \leq A_{\nu}, \nu=1,2 \\
\Leftrightarrow & g_{\nu}\left(A_{\nu}^{-1 / d_{\nu}}, A_{\nu}^{-1 / d_{\nu}} x, A_{\nu}^{-1 / d_{\nu}} y\right) \leq 1, \nu=1,2 \\
\Leftrightarrow & A_{\nu}^{-1 / d_{\nu}} x \leq \xi_{\nu}\left(A_{\nu}^{-1 / d_{\nu}}\right), A_{\nu}^{-1 / d_{\nu}} y \leq y_{\nu}\left(A_{\nu}^{-1 / d_{\nu}}, A_{\nu}^{-1 / d_{\nu}} x\right), \nu=1,2 \\
\Leftrightarrow & x \leq \varrho_{A_{1}, A_{2}}, y \leq f_{A_{1}, A_{2}}(x) .
\end{aligned}
$$

Now it is clear that $R\left(A_{1}, A_{2}\right)$ is the number of lattice points below the graph of $f_{A_{1}, A_{2}}$. The function $\delta:\left(0,\left(\widetilde{\xi}_{1} \widetilde{\xi}_{2}^{-1}\right)^{d_{2}}\right] \rightarrow \mathbb{R}$ with

$$
\delta(C):=\min \left\{\widetilde{y}_{1}(x)-C^{1 / d_{2}} \widetilde{y}_{2}\left(C^{-1 / d_{2}} x\right) \mid 0 \leq x \leq C^{1 / d_{2}} \widetilde{\xi}_{2}\right\}
$$

is continuous with $\lim _{C \rightarrow 0} \delta(C)=\widetilde{y}_{1}(0)>0$ and $\delta\left(\left(\widetilde{\xi}_{1} \widetilde{\xi}_{2}^{-1}\right)^{d_{2}}\right) \leq \widetilde{y}_{1}\left(\widetilde{\xi}_{1}\right)-$ $\widetilde{\xi}_{1} \widetilde{\xi}_{2}^{-1} \widetilde{y}_{2}\left(\widetilde{\xi}_{2}\right)=0$. Choose $0<C_{0} \leq\left(\widetilde{\xi}_{1} \widetilde{\xi}_{2}^{-1}\right)^{d_{2}}$ minimal with $\delta\left(C_{0}\right)=0$ and fix $0<x_{0}<x_{1}<C_{0}^{1 / d_{2}} \widetilde{\xi}_{2}$ with $h^{\prime}\left(x_{0}\right) \neq 0$. Set $C_{1}:=\left(x_{1} \widetilde{\xi}_{2}^{-1}\right)^{d_{2}}<C_{0}$. Then for $0<C \leq C_{1}, 0 \leq x \leq C^{1 / d_{2}} \widetilde{\xi}_{2}$ we have

$$
\widetilde{y}_{1}(x)-C^{1 / d_{2}} \widetilde{y}_{2}\left(C^{-1 / d_{2}} x\right) \geq \delta(C) \geq \min \left\{\delta\left(C^{\prime}\right) \mid 0 \leq C^{\prime} \leq C_{1}\right\}=: K_{1}>0 \text {. }
$$

So for $A_{1}, A_{2} \geq K, C:=A_{2} A_{1}^{-d_{2} / d_{1}} \leq C_{1}, 0 \leq x \leq \varrho_{A_{1}, A_{2}}$, with $\tau_{\nu}:=$ $A_{\nu}^{-1 / d_{\nu}}$, we have

$$
A_{2}^{1 / d_{2}} y_{2}\left(\tau_{2}, \tau_{2} x\right) \leq A_{1}^{1 / d_{1}} C^{1 / d_{2}} \widetilde{y}_{2}\left(C^{-1 / d_{2}} \tau_{1} x\right) \leq A_{1}^{1 / d_{1}}\left(\widetilde{y}_{1}\left(\tau_{1} x\right)-K_{1}\right)
$$


In particular $\widetilde{y}_{1}\left(\tau_{1} x\right) \geq K_{1}>0$ and so $\tau_{1} x \leq \widetilde{\xi}_{1}-K_{3}$ with some constant $K_{3}>0$. Now $y_{1}$ is $C^{\infty}$ on an open neighbourhood of $\left[0, \tau_{0}\right] \times\left[0, \widetilde{\xi}_{1}-K_{3}\right]$ for sufficiently small $\tau_{0}$. The mean value theorem gives $y_{1}\left(\tau_{1}, \tau_{1} x\right)=\widetilde{y}_{1}\left(\tau_{1} x\right)+$ $O\left(\tau_{1}\right)$ and so

$$
A_{2}^{1 / d_{2}} y_{2}\left(\tau_{2}, \tau_{2} x\right) \leq A_{1}^{1 / d_{1}}\left(\widetilde{y}_{1}\left(\tau_{1} x\right)-K_{1}\right) \leq A_{1}^{1 / d_{1}} y_{1}\left(\tau_{1}, \tau_{1} x\right)
$$

if $K$ is sufficiently large. It follows that

$$
f_{A_{1}, A_{2}}(x)=A_{2}^{1 / d_{2}} y_{2}\left(\tau_{2}, \tau_{2} x\right) .
$$

Furthermore from $x_{1}<\widetilde{\xi}_{1}$ it follows that

$$
\begin{aligned}
A_{2}^{1 / d_{2}} \xi_{2}\left(\tau_{2}\right) & =A_{2}^{1 / d_{2}}\left(\widetilde{\xi}_{2}+O\left(\tau_{2}\right)\right) \leq A_{1}^{1 / d_{1}} C_{1}^{1 / d_{2}} \widetilde{\xi}_{2}+O(1) \\
& =A_{1}^{1 / d_{1}}\left(x_{1}+O\left(\tau_{1}\right)\right) \leq A_{1}^{1 / d_{1}} \xi_{1}\left(\tau_{1}\right)
\end{aligned}
$$

and consequently

$$
\varrho_{A_{1}, A_{2}}=A_{2}^{1 / d_{2}} \xi_{2}\left(\tau_{2}\right) .
$$

Set $C_{2}:=\max \left\{\left(2 \widetilde{\xi}_{1} \widetilde{\xi}_{2}^{-1}\right)^{d_{2}},\left(2 \widetilde{y}_{1}(0) \widetilde{y}_{2}\left(\widetilde{\xi}_{2} / 2\right)^{-1}\right)^{d_{2}}\right\}$. Then for $C \geq C_{2}, 0 \leq$ $x \leq \widetilde{\xi}_{1}$ we have

$$
\widetilde{y}_{1}(x) \leq \widetilde{y}_{1}(0) \leq C^{1 / d_{2}} \widetilde{y}_{2}\left(\widetilde{\xi}_{2} / 2\right) / 2 \leq C^{1 / d_{2}}\left(\widetilde{y}_{2}\left(C^{-1 / d_{2}} x\right)-\widetilde{y}_{2}\left(\widetilde{\xi}_{2} / 2\right) / 2\right) .
$$

For $A_{1}, A_{2} \geq K, C:=A_{2} A_{1}^{-d_{2} / d_{1}} \geq C_{2}, 0 \leq x \leq \varrho_{A_{1}, A_{2}}$, it follows that $A_{1}^{1 / d_{1}} y_{1}\left(\tau_{1}, \tau_{1} x\right) \leq A_{1}^{1 / d_{1}} \widetilde{y}_{1}\left(\tau_{1} x\right) \leq A_{1}^{1 / d_{1}} C^{1 / d_{2}}\left(\widetilde{y}_{2}\left(C^{-1 / d_{2}} \tau_{1} x\right)-\widetilde{y}_{2}\left(\widetilde{\xi}_{2} / 2\right) / 2\right)$. In particular $\widetilde{y}_{2}\left(C^{-1 / d_{2}} \tau_{1} x\right) \geq \widetilde{y}_{2}\left(\widetilde{\xi}_{2} / 2\right) / 2>0$ and so $\tau_{2} x \leq \widetilde{\xi}_{2}-K_{4}$ for some constant $K_{4}>0$. Therefore

$$
A_{1}^{1 / d_{1}} y_{1}\left(\tau_{1}, \tau_{1} x\right) \leq A_{2}^{1 / d_{2}}\left(\widetilde{y}_{2}\left(\tau_{2} x\right)+O\left(\tau_{2}\right)\right)=A_{2}^{1 / d_{2}} y_{2}\left(\tau_{2}, \tau_{2} x\right)
$$

for sufficiently large $K$ and so

$$
f_{A_{1}, A_{2}}(x)=A_{1}^{1 / d_{1}} y_{1}\left(\tau_{1}, \tau_{1} x\right) .
$$

Furthermore

$$
\begin{aligned}
A_{1}^{1 / d_{1}} \xi_{1}\left(\tau_{1}\right) & =A_{1}^{1 / d_{1}}\left(\widetilde{\xi}_{1}+O\left(\tau_{1}\right)\right) \leq A_{2}^{1 / d_{2}} C_{2}^{-1 / d_{2}} \widetilde{\xi}_{1}+O(1) \\
& \leq A_{2}^{1 / d_{2}} \widetilde{\xi}_{2} / 2+O(1) \leq A_{2}^{1 / d_{2}} \xi_{2}\left(\tau_{2}\right)
\end{aligned}
$$

and consequently

$$
\varrho_{A_{1}, A_{2}}=A_{1}^{1 / d_{1}} \xi_{1}\left(\tau_{1}\right) .
$$

It remains to analyse the range $C_{1} \leq C \leq C_{2}$. The functions $\widetilde{y}_{\nu}$ can be continued holomorphically to regions of the form

$$
G_{\nu}:=\left\{z \in \mathbb{C}\left|-\varepsilon<\Re z<\widetilde{\xi}_{\nu}+\varepsilon,\right| \Im z \mid<\varepsilon, z \notin\left[\widetilde{\xi}_{\nu}, \infty\right)\right\}
$$


with at most an ordinary algebraic singularity of order $\leq d_{\nu}$ at $\widetilde{\xi}_{\nu}$. This follows from general theorems on algebraic functions (for example [1], Chapter 8.2). Therefore $h^{\prime}$ is holomorphic on $G_{1}$ with at most an algebraic pole at $\widetilde{\xi}_{1}$ and consequently in $\left(0, \widetilde{\xi}_{1}\right)$ it has only a finite number of ze$\operatorname{ros} \xi_{1}<\ldots<\xi_{r-1}$ with $r \in \mathbb{N}$. Define $\xi_{0}:=0, \xi_{r}:=\widetilde{\xi}_{1}$. Let $n_{\varrho} \in$ $\mathbb{N}_{0}$ be the order of the zero $\xi_{\varrho}$ of $h^{\prime}$. For each $1 \leq \varrho \leq r$ the function $h \uparrow\left[\xi_{\varrho-1}, \xi_{\varrho}\right]$ is strictly monotonic and continuous. Therefore it has an inverse $k_{\varrho}:\left[a_{\varrho}, b_{\varrho}\right] \rightarrow\left[\xi_{\varrho-1}, \xi_{\varrho}\right]$ which is strictly monotonic and continuous. For $A_{1}, A_{2} \geq K, C:=A_{2} A_{1}^{-d_{2} / d_{1}} \in\left[C_{1}, C_{2}\right]$, we have

$$
\begin{gathered}
x_{0}<x_{1}<C_{0}^{1 / d_{2}} \widetilde{\xi}_{2} \leq \widetilde{\xi}_{1}, \\
C^{-1 / d_{2}} x_{0} \leq C_{1}^{-1 / d_{2}} x_{0}=x_{0} x_{1}^{-1} \widetilde{\xi}_{2}=: x_{2}<\widetilde{\xi}_{2}
\end{gathered}
$$

and consequently

$$
\begin{aligned}
A_{1}^{-1 / d_{1}} \varrho_{A_{1}, A_{2}} & =\min \left\{\xi_{1}\left(\tau_{1}\right), C^{1 / d_{2}} \xi_{2}\left(\tau_{2}\right)\right\} \\
& =\min \left\{\widetilde{\xi}_{1}+O\left(\tau_{1}\right), C^{1 / d_{2}}\left(\widetilde{\xi}_{2}+O\left(\tau_{2}\right)\right)\right\} \geq x_{0}
\end{aligned}
$$

for sufficiently large $K$. Furthermore

$$
\begin{aligned}
y_{0} & :=y_{0, A_{1}, A_{2}}:=A_{1}^{-1 / d_{1}} f_{A_{1}, A_{2}}\left(A_{1}^{1 / d_{1}} x_{0}\right) \\
& =\min \left\{\widetilde{y}_{1}\left(x_{0}\right)+O\left(\tau_{1}\right), C^{1 / d_{2}}\left(\widetilde{y}_{2}\left(C^{-1 / d_{2}} x_{0}\right)+O\left(\tau_{2}\right)\right)\right\} .
\end{aligned}
$$

So there are constants $y_{1}, y_{2}$ with

$$
\begin{aligned}
y_{0} & \leq \widetilde{y}_{1}\left(x_{0}\right)+O\left(\tau_{1}\right) \leq y_{1}<\widetilde{\eta}_{1}, \\
C^{-1 / d_{2}} y_{0} & \leq \widetilde{y}_{2}\left(C^{-1 / d_{2}} x_{0}\right)+O\left(\tau_{2}\right) \\
& \leq \widetilde{y}_{2}\left(C_{2}^{-1 / d_{2}} x_{0}\right)+O\left(\tau_{2}\right) \leq y_{2}<\widetilde{\eta}_{2}
\end{aligned}
$$

for sufficiently large $K$.

Lemma 2.2. Let $1 \leq \varrho \leq r, \xi_{\varrho-1} \leq \bar{x} \leq \xi_{\varrho}, \bar{x}<\widetilde{\xi}_{1}, 0<\overline{\bar{x}}<\widetilde{\xi}_{2}$, $\bar{C}_{2}>\bar{C}_{1}>0$. There are constants $\varepsilon, K>0$ with the property: For $A_{1}, A_{2} \geq$ $K, C:=A_{2} A_{1}^{-d_{2} / d_{1}} \in\left[\bar{C}_{1}, \bar{C}_{2}\right],\left|C-a_{\varrho}\right|,\left|C-b_{\varrho}\right| \geq A_{1}^{-\varepsilon}, x \in\left[\xi_{\varrho-1}, \bar{x}\right]$, $x \leq C^{1 / d_{2}} \overline{\bar{x}}$, and $\left|x-k_{\varrho}(C)\right| \geq A_{1}^{-50 /\left(73 d_{1}\right)}$ if $C \in\left[a_{\varrho}, b_{\varrho}\right]$ then

$$
f_{A_{1}, A_{2}}\left(A_{1}^{1 / d_{1}} x\right)= \begin{cases}A_{1}^{1 / d_{1}} y_{1}\left(\tau_{1}, x\right), & h(x)<C, \\ A_{2}^{1 / d_{2}} y_{2}\left(\tau_{2}, C^{-1 / d_{2}} x\right), & h(x) \geq C .\end{cases}
$$

Proof. From the mean value theorem it follows that, with $\zeta$ between $\widetilde{y}_{1}(x)$ and $C^{1 / d_{2}} \widetilde{y}_{2}\left(C^{-1 / d_{2}} x\right)$,

$$
\begin{aligned}
|h(x)-C| & =\left|\widetilde{g}_{2}\left(x, \widetilde{y}_{1}(x)\right)-C \widetilde{g}_{2}\left(C^{-1 / d_{2}} x, \widetilde{y}_{2}\left(C^{-1 / d_{2}} x\right)\right)\right| \\
& =\left|\widetilde{y}_{1}(x)-C^{1 / d_{2}} \widetilde{y}_{2}\left(C^{-1 / d_{2}} x\right)\right| \cdot\left|\widetilde{g}_{2 y}(x, \zeta)\right| \\
& \ll\left|\widetilde{y}_{1}(x)-C^{1 / d_{2}} \widetilde{y}_{2}\left(C^{-1 / d_{2}} x\right)\right| .
\end{aligned}
$$


Assume first $C \in\left[a_{\varrho}, b_{\varrho}\right]$. Define $y:=k_{\varrho}(C) \in\left[\xi_{\varrho-1}, \xi_{\varrho}\right]$. Assume $y>$ $\left(\xi_{\varrho-1}+\xi_{\varrho}\right) / 2$.

CASE 1: $\varrho=r, y>\left(\bar{x}+\xi_{\varrho}\right) / 2$. The monotonicity of $h \uparrow\left[\xi_{\varrho-1}, \xi_{\varrho}\right]$ together with (2.10) gives

$$
\begin{aligned}
\left|\widetilde{y}_{1}(x)-C^{1 / d_{2}} \widetilde{y}_{2}\left(C^{-1 / d_{2}} x\right)\right| & \gg|h(x)-C|=|h(x)-h(y)| \\
& \geq\left|h(\bar{x})-h\left(\left(\bar{x}+\widetilde{\xi}_{1}\right) / 2\right)\right| \gg 1 .
\end{aligned}
$$

CASE 2: $\varrho<r$ or $y \leq\left(\bar{x}+\xi_{\varrho}\right) / 2$. Taylor's formula gives

$$
h(x)-C=h(x)-h(y)=h^{\prime}(y)(x-y)+O\left(|x-y|^{2}\right) .
$$

In the case $\varrho=r,\left(\xi_{\varrho-1}+\xi_{\varrho}\right) / 2<y \leq\left(\bar{x}+\xi_{\varrho}\right) / 2$, we have

$$
\left|h^{\prime}(y)\right| \asymp 1 \asymp\left|y-\xi_{\varrho}\right|^{n_{\varrho}} .
$$

In the case $\varrho<r$ the function $h^{\prime}$ has a zero of order $n_{\varrho}$ at $\xi_{\varrho}$ and is nonzero on $\left[\left(\xi_{\varrho-1}+\xi_{\varrho}\right) / 2, \xi_{\varrho}\right)$. This gives $\left|h^{\prime}(y)\right| \asymp\left|y-\xi_{\varrho}\right|^{n_{\varrho}}$ again. So in Case 2 we have

$$
\frac{|h(x)-C|}{\left|x-k_{\varrho}(C)\right|}=\left|h^{\prime}(y)+O(|x-y|)\right| \asymp\left|y-\xi_{\varrho}\right|^{n_{\varrho}}
$$

if $|x-y| \leq \delta\left|y-\xi_{\varrho}\right|^{n_{\varrho}}$. Here $\delta>0$ depends only on $h \uparrow\left[\xi_{\varrho-1}, \xi_{\varrho}\right]$.

CASE 2.1: $|x-y| \leq \delta\left|y-\xi_{\varrho}\right|^{n_{e}}$. With (2.10) it follows that

$$
\left|\widetilde{y}_{1}(x)-C^{1 / d_{2}} \widetilde{y}_{2}\left(C^{-1 / d_{2}} x\right)\right| \gg\left|x-k_{\varrho}(C)\right| \cdot\left|y-\xi_{\varrho}\right|^{n_{\varrho}} .
$$

CASE 2.2: $|x-y|>\delta\left|y-\xi_{\varrho}\right|^{n_{\varrho}}$. Then $x \notin\left[x_{1}, x_{2}\right], x_{1 / 2}:=y \mp \delta\left|y-\xi_{\varrho}\right|^{n_{\varrho}}$. In the case $x<x_{1}$ it follows from the monotonicity of $h \uparrow\left[\xi_{\varrho-1}, \xi_{\varrho}\right]$ that

$$
|h(x)-h(y)| \geq\left|h\left(x_{1}\right)-h(y)\right| \gg\left|x_{1}-k_{\varrho}(C)\right| \cdot\left|y-\xi_{\varrho}\right|^{n_{\varrho}}=\delta\left|y-\xi_{\varrho}\right|^{2 n_{\varrho}}
$$

as in (2.12). The same is true in the case $x>x_{2}$. So in Case 2.2 we have

$$
\left|\widetilde{y}_{1}(x)-C^{1 / d_{2}} \widetilde{y}_{2}\left(C^{-1 / d_{2}} x\right)\right| \gg\left|y-\xi_{\varrho}\right|^{2 n_{\varrho}} .
$$

In the case $\varrho<r$ Taylor's formula gives

$$
h(y)-h\left(\xi_{\varrho}\right)=\frac{h^{\left(n_{\varrho}+1\right)}\left(\xi_{\varrho}\right)}{\left(n_{\varrho}+1\right) !}\left(y-\xi_{\varrho}\right)^{n_{\varrho}+1}\left(1+O\left(\left|y-\xi_{\varrho}\right|\right)\right) .
$$

With $\delta^{\prime}>0$ depending only on $h \uparrow\left[\xi_{\varrho-1}, \xi_{\varrho}\right]$ it follows that, for $\left|y-\xi_{\varrho}\right| \leq \delta^{\prime}$,

$$
\left|y-\xi_{\varrho}\right|^{n_{\varrho}+1} \asymp\left|h(y)-h\left(\xi_{\varrho}\right)\right|=\left|C-h\left(\xi_{\varrho}\right)\right|,
$$

and for $\left|y-\xi_{\varrho}\right|>\delta^{\prime}$,

$$
\left|y-\xi_{\varrho}\right|^{n_{\varrho}+1} \geq \delta^{n_{\varrho}+1} \gg 1 \gg\left|C-h\left(\xi_{\varrho}\right)\right| .
$$

The last estimate is true also in the case $\varrho=r, y \leq\left(\bar{x}+\xi_{\varrho}\right) / 2$. 
Taking $\varepsilon:=23 /\left(73 d_{1}\right)$ it follows in Case 2 from (2.13) and (2.14) and $h\left(\xi_{\varrho}\right) \in\left\{a_{\varrho}, b_{\varrho}\right\}$ that

$$
\begin{aligned}
& \left|\widetilde{y}_{1}(x)-C^{1 / d_{2}} \widetilde{y}_{2}\left(C^{-1 / d_{2}} x\right)\right| \\
& \quad \gg \min \left\{\left|x-k_{\varrho}(C)\right| \cdot\left|y-\xi_{\varrho}\right|^{n_{\varrho}},\left|y-\xi_{\varrho}\right|^{2 n_{\varrho}}\right\} \\
& \quad \gg \min \left\{A_{1}^{-50 /\left(73 d_{1}\right)}\left|C-h\left(\xi_{\varrho}\right)\right|^{n_{\varrho} /\left(n_{\varrho}+1\right)},\left|C-h\left(\xi_{\varrho}\right)\right|^{2 n_{\varrho} /\left(n_{\varrho}+1\right)}\right\} \\
& \quad \gg \min \left\{A_{1}^{-50 /\left(73 d_{1}\right)-\varepsilon n_{\varrho} /\left(n_{\varrho}+1\right)}, A_{1}^{-2 \varepsilon n_{\varrho} /\left(n_{\varrho}+1\right)}\right\} \gg A_{1}^{-\kappa}
\end{aligned}
$$

with some constant $0<\kappa<1 / d_{1}$. By (2.11) this estimate also holds in Case 1. Under the assumption $y \leq\left(\xi_{\varrho-1}+\xi_{\varrho}\right) / 2$ the same arguments can be simplified somewhat.

In the case $C \notin\left[a_{\varrho}, b_{\varrho}\right]$ it follows from (2.10) that

$$
\left|\widetilde{y}_{1}(x)-C^{1 / d_{2}} \widetilde{y}_{2}\left(C^{-1 / d_{2}} x\right)\right| \gg \min \left\{\left|a_{\varrho}-C\right|,\left|b_{\varrho}-C\right|\right\} \gg A_{1}^{-\varepsilon},
$$

which also gives (2.15).

The equivalence

$$
\begin{aligned}
& \widetilde{y}_{1}(x) \geq C^{1 / d_{2}} \widetilde{y}_{2}\left(C^{-1 / d_{2}} x\right) \\
& \Leftrightarrow \widetilde{g}_{2}\left(C^{-1 / d_{2}} x, C^{-1 / d_{2}} \widetilde{y}_{1}(x)\right) \geq 1 \Leftrightarrow h(x) \geq C
\end{aligned}
$$

follows from the definitions. As $x \leq \bar{x}<\widetilde{\xi}_{1}, C^{-1 / d_{2}} x \leq \overline{\bar{x}}<\widetilde{\xi}_{2}$ it follows from the mean value theorem that

$$
y_{1}\left(\tau_{1}, x\right)=\widetilde{y}_{1}(x)+O\left(\tau_{1}\right), \quad y_{2}\left(\tau_{2}, C^{-1 / d_{2}} x\right)=\widetilde{y}_{2}\left(C^{-1 / d_{2}} x\right)+O\left(\tau_{2}\right) .
$$

If $h(x)<C$ then by $(2.16)$ and $(2.15)$,

$$
\begin{aligned}
y_{1}\left(\tau_{1}, x\right)-C^{1 / d_{2}} y_{2}\left(\tau_{2}, C^{-1 / d_{2}} x\right) & =\widetilde{y}_{1}(x)-C^{1 / d_{2}} \widetilde{y}_{2}\left(C^{-1 / d_{2}} x\right)+O\left(\tau_{1}\right) \\
& \leq-K_{4} A_{1}^{-\kappa}+K_{5} A_{1}^{-1 / d_{1}}<0
\end{aligned}
$$

for sufficiently large $K$ where $K_{4}, K_{5}>0$ are constants independent of $A_{1}, A_{2}$. If $h(x) \geq C$ the same reasoning gives

$$
y_{1}\left(\tau_{1}, x\right)-C^{1 / d_{2}} y_{2}\left(\tau_{2}, C^{-1 / d_{2}} x\right)>0 .
$$

From this (2.9) follows.

3. Decomposition of the lattice point set. For $A_{1}, A_{2} \geq K, C:=$ $A_{2} A_{1}^{-d_{2} / d_{1}} \leq C_{1}$ it follows from (2.2), (2.3) and (2.4) that

$$
R\left(A_{1}, A_{2}\right)=\#\left\{(x, y) \in \mathbb{N}^{2} \mid x \leq A_{2}^{1 / d_{2}} \xi_{2}\left(\tau_{2}\right), y \leq A_{2}^{1 / d_{2}} y_{2}\left(\tau_{2}, \tau_{2} x\right)\right\} .
$$

If $C \geq C_{2}$ it follows from (2.5) and (2.6) that

$$
R\left(A_{1}, A_{2}\right)=\#\left\{(x, y) \in \mathbb{N}^{2} \mid x \leq A_{1}^{1 / d_{1}} \xi_{1}\left(\tau_{1}\right), y \leq A_{1}^{1 / d_{1}} y_{1}\left(\tau_{1}, \tau_{1} x\right)\right\} .
$$

So in these cases the problem of evaluating $R\left(A_{1}, A_{2}\right)$ asymptotically involves only one polynomial. This situation was investigated in [9]. There 
the error estimates are more precise than in this paper and an explicit description of the terms $T_{\nu}$ and $U_{\nu}$ is possible. Therefore in the remainder of this paper the range $C \in\left[C_{1}, C_{2}\right]$ is investigated. Then

$$
R\left(A_{1}, A_{2}\right)=R^{\dagger}\left(A_{1}, A_{2}\right)+R^{\#}\left(A_{1}, A_{2}\right)-\left[A_{1}^{1 / d_{1}} y_{0, A_{1}, A_{2}}\right]\left[A_{1}^{1 / d_{1}} x_{0}\right]
$$

with

$$
\begin{aligned}
R^{\dagger}\left(A_{1}, A_{2}\right) & =\#\left\{(x, y) \in \mathbb{N}^{2} \mid x \leq A_{1}^{1 / d_{1}} x_{0}, y \leq f_{A_{1}, A_{2}}(x)\right\}, \\
R^{\#}\left(A_{1}, A_{2}\right) & =\#\left\{(x, y) \in \mathbb{N}^{2} \mid y \leq A_{1}^{1 / d_{1}} y_{0, A_{1}, A_{2}}, x \leq f_{A_{1}, A_{2}}^{-1}(y)\right\} .
\end{aligned}
$$

From $h^{\prime}\left(x_{0}\right) \neq 0$ it follows that there is some $1 \leq \varrho_{0} \leq r$ with $\xi_{\varrho_{0}-1}<x_{0}<$ $\xi_{\varrho_{0}}$. Take $\varrho=\varrho_{0}, x=\bar{x}=x_{0}, \overline{\bar{x}}=x_{2}, \bar{C}_{1}=C_{1}, \bar{C}_{2}=C_{2}$ in Lemma 2.2. From (2.7) it follows that for $A_{1}, A_{2} \geq K, C:=A_{2} A_{1}^{-d_{2} / d_{1}} \in\left[C_{1}, C_{2}\right]$, $\left|C-a_{\varrho_{0}}\right|,\left|C-b_{\varrho_{0}}\right| \geq A_{1}^{-\varepsilon}$ and $\left|x_{0}-k_{\varrho_{0}}(C)\right| \geq A_{1}^{-50 /\left(73 d_{1}\right)}$ if $C \in\left[a_{\varrho_{0}}, b_{\varrho_{0}}\right]$ then

$$
\begin{aligned}
& y_{0, A_{1}, A_{2}} \\
= & \begin{cases}y_{1}\left(\tau_{1}, x_{0}\right)=\widetilde{y}_{1}\left(x_{0}\right)+O\left(\tau_{1}\right), & C>h\left(x_{0}\right), \\
C^{1 / d_{2}} y_{2}\left(\tau_{2}, C^{-1 / d_{2}} x_{0}\right)=C^{1 / d_{2}} \widetilde{y}_{2}\left(C^{-1 / d_{2}} x_{0}\right)+O\left(\tau_{1}\right), & C \leq h\left(x_{0}\right) .\end{cases}
\end{aligned}
$$

From $k_{\varrho_{0}}^{\prime}\left(h\left(x_{0}\right)\right)=h^{\prime}\left(x_{0}\right)^{-1} \neq 0$ it follows that for $C \in\left[a_{\varrho_{0}}, b_{\varrho_{0}}\right]$ by Taylor's theorem we have

$C-h\left(x_{0}\right)=h\left(k_{\varrho_{0}}(C)\right)-h\left(x_{0}\right)=h^{\prime}\left(x_{0}\right)\left(k_{\varrho_{0}}(C)-x_{0}\right)\left(1+O\left(\left|k_{\varrho_{0}}(C)-x_{0}\right|\right)\right)$ and consequently $\left|C-h\left(x_{0}\right)\right| \asymp\left|k_{\varrho_{0}}(C)-x_{0}\right|$ for $k_{\varrho_{0}}(C)$ near $x_{0}$. In the opposite case the same holds by the monotonicity of $h \uparrow\left[\xi_{\varrho_{0}-1}, \xi_{\varrho_{0}}\right]$. Therefore there are constants $\varepsilon_{0}, K>0$ so that (3.2) holds for $A_{1}, A_{2} \geq K, C:=$ $A_{2} A_{1}^{-d_{2} / d_{1}} \in\left[C_{1}, C_{2}\right],\left|C-a_{\varrho_{0}}\right|,\left|C-b_{\varrho_{0}}\right|,\left|C-h\left(x_{0}\right)\right| \geq A_{1}^{-\varepsilon_{0}}$.

$R^{\dagger}\left(A_{1}, A_{2}\right)$ and $R^{\#}\left(A_{1}, A_{2}\right)$ are defined in the same way but with $x$ and $y$ interchanged. The only asymmetry is that $x_{0}$ is constant whereas $y_{0}, A_{1}, A_{2}$ depends on $A_{1}, A_{2}$. Therefore the following notation is introduced which covers both cases: Let $\bar{C}_{2}>\bar{C}_{1}>0$ and $0<\bar{z}<\widetilde{\xi}_{1}, 0<\overline{\bar{z}}<\widetilde{\xi}_{2}$. Let $z:[K, \infty)^{2} \rightarrow \mathbb{R}^{+}$be a function with

$$
z\left(A_{1}, A_{2}\right) \leq \bar{z}, \quad C^{-1 / d_{2}} z\left(A_{1}, A_{2}\right) \leq \overline{\bar{z}}
$$

for $A_{1}, A_{2} \geq K, C:=A_{2} A_{1}^{-d_{2} / d_{1}} \in\left[\bar{C}_{1}, \bar{C}_{2}\right]$. Define

$$
R^{*}\left(A_{1}, A_{2}\right):=\#\left\{(x, y) \in \mathbb{N}^{2} \mid x \leq A_{1}^{1 / d_{1}} z\left(A_{1}, A_{2}\right), y \leq f_{A_{1}, A_{2}}(x)\right\} .
$$

From (2.7) and (2.8) it follows that both $R^{\dagger}$ and $R^{\#}$ are of this type. Then $R^{*}\left(A_{1}, A_{2}\right)=\sum_{\varrho=1}^{r} R_{\varrho}^{*}\left(A_{1}, A_{2}\right)$ with

$$
\begin{array}{r}
R_{\varrho}^{*}\left(A_{1}, A_{2}\right):=\#\left\{(x, y) \in \mathbb{Z}^{2} \mid A_{1}^{1 / d_{1}} \xi_{\varrho-1}<x \leq A_{1}^{1 / d_{1}} \min \left\{\xi_{\varrho}, z\left(A_{1}, A_{2}\right)\right\},\right. \\
\left.0<y \leq f_{A_{1}, A_{2}}(x)\right\} .
\end{array}
$$


The following lemma gives a reduction of $R_{\varrho}^{*}$ to the case where only one algebraic curve is involved.

Lemma 3.1. Let $1 \leq \varrho \leq r$. Then for $A_{1}, A_{2} \geq K, C:=A_{2} A_{1}^{-d_{2} / d_{1}} \in$ $\left[\bar{C}_{1}, \bar{C}_{2}\right],\left|C-a_{\varrho}\right|,\left|C-b_{\varrho}\right| \geq A_{1}^{-\varepsilon}$ we have:

- in the case $C \in\left[a_{\varrho}, b_{\varrho}\right], h \uparrow\left[\xi_{\varrho-1}, \xi_{\varrho}\right]$ increasing with $z_{\varrho}\left(A_{1}, A_{2}\right):=$ $\min \left\{k_{\varrho}(C), z\left(A_{1}, A_{2}\right)\right\}$ :

$$
\begin{array}{r}
R_{\varrho}^{*}\left(A_{1}, A_{2}\right)=\#\left\{(x, y) \in \mathbb{Z}^{2} \mid A_{1}^{1 / d_{1}} \xi_{\varrho-1}<x \leq A_{1}^{1 / d_{1}} z_{\varrho}\left(A_{1}, A_{2}\right),\right. \\
\left.0<y \leq A_{1}^{1 / d_{1}} y_{1}\left(\tau_{1}, \tau_{1} x\right)\right\} \\
+\#\left\{(x, y) \in \mathbb{Z}^{2} \mid A_{1}^{1 / d_{1}} k_{\varrho}(C)<x \leq A_{1}^{1 / d_{1}} \min \left\{\xi_{\varrho}, z\left(A_{1}, A_{2}\right)\right\},\right. \\
\left.0<y \leq A_{2}^{1 / d_{2}} y_{2}\left(\tau_{2}, \tau_{2} x\right)\right\}+O\left(A_{1}^{46 /\left(73 d_{1}\right)}\right) ;
\end{array}
$$

- in the case $C \in\left[a_{\varrho}, b_{\varrho}\right], h \uparrow\left[\xi_{\varrho-1}, \xi_{\varrho}\right]$ decreasing:

$$
\begin{array}{r}
R_{\varrho}^{*}\left(A_{1}, A_{2}\right)=\#\left\{(x, y) \in \mathbb{Z}^{2} \mid A_{1}^{1 / d_{1}} \xi_{\varrho-1}<x \leq A_{1}^{1 / d_{1}} z_{\varrho}\left(A_{1}, A_{2}\right),\right. \\
\left.0<y \leq A_{2}^{1 / d_{2}} y_{2}\left(\tau_{2}, \tau_{2} x\right)\right\} \\
+\#\left\{(x, y) \in \mathbb{Z}^{2} \mid A_{1}^{1 / d_{1}} k_{\varrho}(C)<x \leq A_{1}^{1 / d_{1}} \min \left\{\xi_{\varrho}, z\left(A_{1}, A_{2}\right)\right\},\right. \\
\left.0<y \leq A_{1}^{1 / d_{1}} y_{1}\left(\tau_{1}, \tau_{1} x\right)\right\}+O\left(A_{1}^{46 /\left(73 d_{1}\right)}\right) ;
\end{array}
$$

- in the case $C<a_{\varrho}$ :

$$
\begin{array}{r}
R_{\varrho}^{*}\left(A_{1}, A_{2}\right)=\#\left\{(x, y) \in \mathbb{Z}^{2} \mid A_{1}^{1 / d_{1}} \xi_{\varrho-1}<x \leq A_{1}^{1 / d_{1}} \min \left\{\xi_{\varrho}, z\left(A_{1}, A_{2}\right)\right\},\right. \\
\left.0<y \leq A_{2}^{1 / d_{2}} y_{2}\left(\tau_{2}, \tau_{2} x\right)\right\} ;
\end{array}
$$

- in the case $C>b_{\varrho}$ :

$$
\begin{array}{r}
R_{\varrho}^{*}\left(A_{1}, A_{2}\right)=\#\left\{(x, y) \in \mathbb{Z}^{2} \mid A_{1}^{1 / d_{1}} \xi_{\varrho-1}<x \leq A_{1}^{1 / d_{1}} \min \left\{\xi_{\varrho}, z\left(A_{1}, A_{2}\right)\right\},\right. \\
\left.0<y \leq A_{1}^{1 / d_{1}} y_{1}\left(\tau_{1}, \tau_{1} x\right)\right\} .
\end{array}
$$

Proof. Only the case $z\left(A_{1}, A_{2}\right)>\xi_{\varrho-1}$ is of interest. Assume first $C \in\left[a_{\varrho}, b_{\varrho}\right], h \uparrow\left[\xi_{\varrho-1}, \xi_{\varrho}\right]$ increasing. For $\xi_{\varrho-1} \leq x \leq \min \left\{\xi_{\varrho}, z\left(A_{1}, A_{2}\right)\right\}$, $\left|x-k_{\varrho}(C)\right| \geq \tau_{1}^{50 / 73}$ it follows from Lemma 2.2 with $\bar{x}:=\min \left\{\xi_{\varrho}, \bar{z}\right\}, \overline{\bar{x}}:=\overline{\bar{z}}$ that

$$
f_{A_{1}, A_{2}}\left(A_{1}^{1 / d_{1}} x\right)= \begin{cases}A_{2}^{1 / d_{2}} y_{2}\left(\tau_{2}, C^{-1 / d_{2}} x\right), & x>k_{\varrho}(C), \\ A_{1}^{1 / d_{1}} y_{1}\left(\tau_{1}, x\right), & x<k_{\varrho}(C) .\end{cases}
$$

Consequently,

$$
\begin{array}{r}
R_{\varrho}^{*}\left(A_{1}, A_{2}\right) \\
=\#\left\{(x, y) \in \mathbb{Z}^{2} \mid A_{1}^{1 / d_{1}} \xi_{\varrho-1}<x \leq A_{1}^{1 / d_{1}} \min \left\{k_{\varrho}(C)-\tau_{1}^{50 / 73}, z\left(A_{1}, A_{2}\right)\right\},\right. \\
\left.0<y \leq A_{1}^{1 / d_{1}} y_{1}\left(\tau_{1}, \tau_{1} x\right)\right\}
\end{array}
$$




$$
\begin{array}{r}
+\#\left\{(x, y) \in \mathbb{Z}^{2} \mid A_{1}^{1 / d_{1}} \max \left\{\xi_{\varrho-1}, k_{\varrho}(C)-\tau_{1}^{50 / 73}\right\}<x\right. \\
\left.\leq A_{1}^{1 / d_{1}} \min \left\{k_{\varrho}(C)+\tau_{1}^{50 / 73}, z\left(A_{1}, A_{2}\right), \xi_{\varrho}\right\}, 0<y \leq f_{A_{1}, A_{2}}(x)\right\} \\
+\#\left\{(x, y) \in \mathbb{Z}^{2} \mid A_{1}^{1 / d_{1}}\left(k_{\varrho}(C)+\tau_{1}^{50 / 73}\right)<x \leq A_{1}^{1 / d_{1}} \min \left\{\xi_{\varrho}, z\left(A_{1}, A_{2}\right)\right\},\right. \\
\left.0<y \leq A_{2}^{1 / d_{2}} y_{2}\left(\tau_{2}, \tau_{2} x\right)\right\} .
\end{array}
$$

For $\max \left\{\xi_{\varrho-1}, k_{\varrho}(C)-\tau_{1}^{50 / 73}\right\} \leq x \leq \min \left\{\xi_{\varrho}, k_{\varrho}(C)+\tau_{1}^{50 / 73}, z\left(A_{1}, A_{2}\right)\right\}$ we have $x=k_{\varrho}(C)+O\left(\tau_{1}^{50 / 73}\right), k_{\varrho}(C) \leq z\left(A_{1}, A_{2}\right)+\tau_{1}^{50 / 73}$ and by Taylor's theorem

$$
\begin{aligned}
y_{1}\left(\tau_{1}, x\right) & =\widetilde{y}_{1}\left(k_{\varrho}(C)\right)+O\left(\tau_{1}^{50 / 73}\right), \\
y_{2}\left(\tau_{2}, C^{-1 / d_{2}} x\right) & =\widetilde{y}_{2}\left(C^{-1 / d_{2}} k_{\varrho}(C)\right)+O\left(\tau_{2}^{50 / 73}\right) .
\end{aligned}
$$

Furthermore

$$
\begin{aligned}
\widetilde{g}_{2}\left(C^{-1 / d_{2}} k_{\varrho}(C), C^{-1 / d_{2}} \widetilde{y}_{1}\left(k_{\varrho}(C)\right)\right) & =C^{-1} \widetilde{g}_{2}\left(k_{\varrho}(C), \widetilde{y}_{1}\left(k_{\varrho}(C)\right)\right) \\
& =C^{-1} h\left(k_{\varrho}(C)\right)=1
\end{aligned}
$$

and consequently

$$
\widetilde{y}_{2}\left(C^{-1 / d_{2}} k_{\varrho}(C)\right)=C^{-1 / d_{2}} \widetilde{y}_{1}\left(k_{\varrho}(C)\right) .
$$

This gives

$$
\begin{aligned}
f_{A_{1}, A_{2}}\left(A_{1}^{1 / d_{1}} x\right) & =A_{1}^{1 / d_{1}} \widetilde{y}_{1}\left(k_{\varrho}(C)\right)+O\left(A_{1}^{23 /\left(73 d_{1}\right)}\right) \\
& =A_{2}^{1 / d_{2}} \widetilde{y}_{2}\left(C^{-1 / d_{2}} k_{\varrho}(C)\right)+O\left(A_{2}^{23 /\left(73 d_{2}\right)}\right) .
\end{aligned}
$$

It follows that

$$
\begin{aligned}
& R_{\varrho}^{*}\left(A_{1}, A_{2}\right) \\
& =\#\left\{(x, y) \in \mathbb{Z}^{2} \mid A_{1}^{1 / d_{1}} \xi_{\varrho-1}<x \leq A_{1}^{1 / d_{1}} z_{\varrho}\left(A_{1}, A_{2}\right),\right. \\
& -\sum_{A_{1}^{1 / d_{1}} \max \left\{k_{\varrho}(C)-\tau_{1}^{50 / 73}, \xi_{\varrho-1}\right\}<x \leq A_{1}^{1 / d_{1}} z_{\varrho}\left(A_{1}, A_{2}\right)}\left[A_{1}^{1 / d_{1}} y_{1}\left(\tau_{1}, \tau_{1} x\right)\right] \\
& +\sum_{A_{1}^{1 / d_{1}} \max \left\{\xi_{\varrho-1}, k_{\varrho}(C)-\tau_{1}^{50 / 73}\right\}<x \leq A_{1}^{1 / d_{1}} z_{\varrho}\left(A_{1}, A_{2}\right)}\left[f_{A_{1}, A_{2}}(x)\right] \\
& +\sum\left[f_{A_{1}, A_{2}}(x)\right] \\
& A_{1}^{1 / d_{1}} \max \left\{k_{\varrho}(C)-\tau_{1}^{50 / 73}, z_{\varrho}\left(A_{1}, A_{2}\right)\right\}<x \leq A_{1}^{1 / d_{1}} \min \left\{k_{\varrho}(C)+\tau_{1}^{50 / 73}, z\left(A_{1}, A_{2}\right), \xi_{\varrho}\right\} \\
& +\#\left\{(x, y) \in \mathbb{Z}^{2} \mid A_{1}^{1 / d_{1}} k_{\varrho}(C)<x \leq A_{1}^{1 / d_{1}} \min \left\{\xi_{\varrho}, z\left(A_{1}, A_{2}\right)\right\}\right. \text {, } \\
& \text { - } \quad \sum \quad\left[A_{2}^{1 / d_{2}} y_{2}\left(\tau_{2}, \tau_{2} x\right)\right] \text {. } \\
& A_{1}^{1 / d_{1}} k_{\varrho}(C)<x \leq A_{1}^{1 / d_{1}} \min \left\{k_{\varrho}(C)+\tau_{1}^{50 / 73}, \xi_{\varrho}, z\left(A_{1}, A_{2}\right)\right\}
\end{aligned}
$$


By (3.3) and (3.5) the difference between the first and the second sum is $O\left(A_{1}^{23 /\left(73 d_{1}\right)} A_{1}^{1 / d_{1}} \tau_{1}^{50 / 73}\right)=O\left(A_{1}^{46 /\left(73 d_{1}\right)}\right)$. The difference between the third and the fourth sum is

$$
\begin{aligned}
& O\left(A_{2}^{23 /\left(73 d_{2}\right)} A_{1}^{1 / d_{1}} \tau_{1}^{50 / 73}\right) \\
& +\sum_{A_{1}^{1 / d_{1}} \max \left\{k_{\varrho}(C)-\tau_{1}^{50 / 73}, z_{\varrho}\left(A_{1}, A_{2}\right)\right\}<x \leq A_{1}^{1 / d_{1}} z_{\varrho}\left(A_{1}, A_{2}\right)}\left[f_{A_{1}, A_{2}}(x)\right] .
\end{aligned}
$$

The sum is zero and the error term is $O\left(A_{1}^{46 /\left(73 d_{1}\right)}\right)$. The case of $h\left\lceil\left[\xi_{\varrho-1}, \xi_{\varrho}\right]\right.$ decreasing is handled in the same way.

Assume now $C<a_{\varrho}$. For $\xi_{\varrho-1} \leq x \leq \min \left\{\xi_{\varrho}, z\left(A_{1}, A_{2}\right)\right\}$ we have $h(x) \geq$ $a_{\varrho}>C$. From Lemma 2.2 it follows that $f_{A_{1}, A_{2}}\left(A_{1}^{1 / d_{1}} x\right)=A_{2}^{1 / d_{2}} y_{2}\left(\tau_{2}\right.$, $\left.C^{-1 / d_{2}} x\right)$. This proves the conclusion of the theorem. The case $C>b_{\varrho}$ is handled in the same way.

4. The case of irrational slope. In this and the next section the following general situation is investigated:

Let $\tau_{0}>0, d \in \mathbb{N}, a, b \in \mathbb{R}$, and let $f: U \rightarrow \mathbb{R}$ be $C^{\infty}$ on the open neighbourhood $U$ of $\left\{(\tau, x) \mid 0 \leq \tau \leq \tau_{0}, a \leq x \leq b\right\}$. Define $\widetilde{f}:=f(0, \cdot)$. For $A \geq \tau_{0}^{-d}, A^{1 / d} a \leq x \leq A^{1 / d} b$, define $f_{A}(x):=A^{1 / d} f\left(A^{-1 / d}, A^{-1 / d} x\right)$. Let $a, b:\left[0, \tau_{0}\right] \rightarrow \mathbb{R}$ be functions with $a(\tau)=a+O(\tau), b(\tau)=b+O(\tau)$ as $\tau \rightarrow 0$.

The argument of this section follows the general line of Müller-Nowak [6]. The main difference is that the estimates are uniform in the variable $\tau$. This gives rise to additional complications.

Lemma 4.1. Let $I \subseteq \mathbb{R}$ be an interval and $f \in C^{\infty}(I)$ with $\widetilde{g}_{\nu}(x, f(x))=1$ for $x \in I$. Then $f^{(k)} \not \equiv 0$ for each $k \in \mathbb{N}_{0}$. In particular $\widetilde{y}_{\nu}^{(k)} \not \equiv 0$ on $\left[0, \widetilde{\xi}_{\nu}\right)$ and $\widetilde{x}_{\nu}^{(k)} \not \equiv 0$ on $\left[0, \widetilde{\eta}_{\nu}\right)$ for each $k \in \mathbb{N}_{0}$.

Proof. Surely $f$ is not constant. If $f^{(k)} \equiv 0$ is assumed for some $k \in \mathbb{N}_{0}$ then let $k$ be minimal with this property. Then $k \geq 2$ and $f(x)=\sum_{\kappa=0}^{k-1} b_{\kappa} x^{\kappa}$ on $I$ with coefficients $b_{\kappa} \in \mathbb{C}$ and $b_{k-1} \neq 0$. Then

$$
\sum_{i+j=d_{\nu}} a_{i j}^{(\nu)} x^{i}\left(\sum_{\kappa=0}^{k-1} b_{\kappa} x^{\kappa}\right)^{j}=1 .
$$

For $k \geq 3$ this gives the contradiction $1=a_{0 d_{\nu}}^{(\nu)}\left(b_{k-1} x^{k-1}\right)^{d_{\nu}}+$ monomials of lower order. Therefore $k=2$ and

$$
\sum_{m=0}^{d_{\nu}} x^{m} \sum_{\substack{0 \leq \iota \leq j \leq d_{\nu} \\
d_{\nu}-\iota=m}} a_{d_{\nu}-j, j}^{(\nu)}\left(\begin{array}{l}
j \\
\iota
\end{array}\right) b_{1}^{j-\iota} b_{0}^{\iota}=1 .
$$


This gives $a_{0 d_{\nu}}^{(\nu)} b_{0}^{d_{\nu}}=1$ and therefore $b_{0} \neq 0$, and for $1 \leq m \leq d_{\nu}$,

$$
\begin{aligned}
0 & =\sum_{j=d_{\nu}-m}^{d_{\nu}} a_{d_{\nu}-j, j}^{(\nu)}\left(\begin{array}{c}
j \\
d_{\nu}-m
\end{array}\right) b_{1}^{j-\left(d_{\nu}-m\right)} b_{0}^{d_{\nu}-m} \\
& =b_{0}^{d_{\nu}-m} \frac{1}{\left(d_{\nu}-m\right) !} \cdot \frac{\partial^{d_{\nu}-m}}{\partial y^{d_{\nu}-m}} \widetilde{g}_{\nu}\left(1, b_{1}\right) .
\end{aligned}
$$

Therefore $b_{1}$ is a zero of $\widetilde{g}_{\nu}(1, y)$ with multiplicity at least $d_{\nu}$ and so $\widetilde{g}_{\nu}(1, y)=$ $a_{0 d_{\nu}}^{(\nu)}\left(y-b_{1}\right)^{d_{\nu}}$. Since the coefficients of $\widetilde{g}_{\nu}$ are real this would imply $b_{1} \in \mathbb{R}$ and $\widetilde{g}_{\nu}(x, y)=a_{0 d_{\nu}}^{(\nu)}\left(y-b_{1} x\right)^{d_{\nu}}$ contrary to assumption (1.2).

LEMmA 4.2. Let $x_{0} \in\left[0, \widetilde{\xi}_{\nu}\right)$ with $\widetilde{y}_{\nu}^{\prime \prime}\left(x_{0}\right)=0$. Then $\widetilde{y}_{\nu}^{\prime}\left(x_{0}\right)$ is algebraic over $\mathbb{Q}$.

Pr o of. Twofold differentiation of $\widetilde{g}_{\nu}\left(x, \widetilde{y}_{\nu}(x)\right)=1$ shows $\left(x_{0}, \widetilde{y}_{\nu}\left(x_{0}\right)\right)$ is a zero of $k:=\widetilde{g}_{\nu}-1$ and $l:=\widetilde{g}_{\nu x x} \widetilde{g}_{\nu y}^{2}-2 \widetilde{g}_{\nu x y} \widetilde{g}_{\nu x} \widetilde{g}_{\nu y}+\widetilde{g}_{\nu y y} \widetilde{g}_{\nu x}^{2}$. Now $l$ is not zero because otherwise $\widetilde{y}_{\nu}^{\prime \prime} \equiv 0$ contrary to Lemma 4.1 . Let $0 \neq b(x) \in \mathbb{Z}[x]$ be the leading coefficient of $l$ as a polynomial in $y$ with coefficients in $\mathbb{Z}[x]$. Let $R(x) \in \mathbb{Z}[x]$ be the resultant of $k$ and $l$ with respect to $y$. Then $R\left(x_{0}\right)=0$ or $b\left(x_{0}\right)=0$ because $k$ has leading coefficient $a_{0 d_{\nu}}^{(\nu)} \neq 0$ with respect to $y$ (van der Waerden [10], p. 104). If $R \neq 0$ then $x_{0} \in \overline{\mathbb{Q}}$. Then $k\left(x_{0}, \cdot\right) \neq 0$ has algebraic coefficients and therefore its zero $\widetilde{y}_{\nu}\left(x_{0}\right)$ is algebraic. Consequently,

$$
\widetilde{y}_{\nu}^{\prime}\left(x_{0}\right)=-\widetilde{g}_{\nu x}\left(x_{0}, \widetilde{y}_{\nu}\left(x_{0}\right)\right) \widetilde{g}_{\nu y}\left(x_{0}, \widetilde{y}_{\nu}\left(x_{0}\right)\right)^{-1} \in \overline{\mathbb{Q}} \text {. }
$$

Now the assumption $R=0$ will be proved contradictory. Then for each $z \in \mathbb{C}$ with $b(z) \neq 0$ the polynomials $k(z, \cdot)$ and $l(z, \cdot)$ would have a common zero. The discriminant $D_{k}(x)$ of $k$ with respect to $y$ is not zero because $D_{k}(0)=(-1)^{d_{\nu}-1}\left(d_{\nu} a_{0 d_{\nu}}^{(\nu)}\right)^{d_{\nu}} \neq 0$. Let $x_{1} \in \mathbb{R}$ with $D_{k}\left(x_{1}\right) \neq 0, b\left(x_{1}\right) \neq 0$. From general theorems on algebraic functions it follows that there is an open disk $U \subseteq \mathbb{C}$ with centre $x_{1}$ where $b$ and $D_{k}$ have no zeros and on which there exist $d_{\nu}$ holomorphic branches $\widetilde{w}_{1}, \ldots, \widetilde{w}_{d_{\nu}}$ of the algebraic function which is defined by $k(z, w)=0$. Twofold differentiation of $k\left(z, \widetilde{w}_{j}(z)\right)=0$ gives $\widetilde{w}_{j}^{\prime \prime}(z)=-\left(l / \widetilde{g}_{\nu y}^{3}\right)\left(z, \widetilde{w}_{j}(z)\right)$ on $U$. Here $\widetilde{g}_{\nu y}\left(z, \widetilde{w}_{j}(z)\right) \neq 0$ for $z \in U$ because $k(z, \cdot)$ has only simple zeros. We have $b(z) \neq 0$ and $\widetilde{w}_{1}(z), \ldots, \widetilde{w}_{d_{\nu}}(z)$ are the zeros of $k(z, \cdot)$. Therefore by assumption there is some $1 \leq j(z) \leq d_{\nu}$ with $l\left(z, \widetilde{w}_{j(z)}(z)\right)=0$ and consequently $\widetilde{w}_{j(z)}^{\prime \prime}(z)=0$. From the identity theorem it follows that $\widetilde{w}_{j}^{\prime \prime}(z)=0$ on $U$ for some $1 \leq j \leq d_{\nu}$. This contradicts Lemma 4.1.

The following lemma is used for parts of the boundary curve on which the curvature does not vanish. 
LEMma 4.3. Let $\widetilde{f}^{\prime \prime}, \widetilde{f}^{\prime \prime \prime}$ be zerofree on $[a, b]$. Then for $A \geq \tau_{0}^{-d}, a \leq a^{\prime}<$ $b^{\prime} \leq b$ we have

$$
\sum_{A^{1 / d} a^{\prime}<n \leq A^{1 / d} b^{\prime}} \psi\left(f_{A}(n)\right) \ll A^{46 /(73 d)}(\log A)^{315 / 146} .
$$

Proof. Define $\tau:=A^{-1 / d}, M:=\left[A^{1 / d}(b-a)\right], T:=A^{1 / d} M$. For $A^{1 / d} a \leq x \leq A^{1 / d} b, 0 \leq \tau \leq \tau_{0}$ the mean value theorem gives

$$
f_{A}^{\prime \prime}(x)=A^{-1 / d} f_{x x}(\tau, \tau x)=A^{-1 / d}\left(\tilde{f}^{\prime \prime}(\tau x)+O(\tau)\right) .
$$

$\tilde{f}^{\prime \prime}$ is zerofree and consequently $\left|\tilde{f}^{\prime \prime}(\tau x)\right| \asymp 1$. For large $A$ this gives $\left|f_{A}^{\prime \prime}(x)\right| \asymp$ $A^{-1 / d} \asymp T M^{-3}$ and similarly $\left|f_{A}^{\prime \prime \prime}(x)\right| \asymp T M^{-4}$. Let $c \in[a, b], M_{c}:=$ $M+\left[A^{1 / d}(c-a)\right]$. Define $h(x):=f_{A}\left(x+\left[A^{1 / d} a\right]-M\right), x \in[M, 2 M]$. Then $\left|h^{\prime \prime}(x)\right| \asymp T M^{-3},\left|h^{\prime \prime \prime}(x)\right| \asymp T M^{-4}, M \leq M_{c} \leq 2 M, T^{1 / 2} \asymp M$, and the discrete Hardy-Littlewood method in the form of [2], Theorem 18.2.2, gives

$$
\sum_{A^{1 / d} a<n \leq A^{1 / d} c} \psi\left(f_{A}(n)\right)=\sum_{M \leq n \leq M_{c}} \psi(h(n))+O(1) \ll A^{46 /(73 d)}(\log A)^{315 / 146}
$$

uniformly in $A$ and $c$. In this theorem $f(\tau, \cdot)$ is assumed to be independent of $\tau$. This is not an essential assumption as was pointed out in [7], Theorem $\mathrm{B}$. Choosing $c=b^{\prime}$ and $c=a^{\prime}$ and subtracting proves the lemma.

The next lemma is used for parts of the boundary curve which do not come too close to points of vanishing curvature.

Lemma 4.4. Let $\widetilde{f}^{\prime}, \widetilde{f}^{\prime \prime}, \widetilde{f}^{\prime \prime \prime}$ be zerofree on $(a, b]$. Let $\mu \in \mathbb{N}$ with $\widetilde{f}^{(k)}(a)$ $=0$ for $2 \leq k \leq \mu+1$ and $\widetilde{f}^{(\mu+2)}(a) \neq 0$. Let $0<\lambda<(\mu+1)^{-1}$ and $\lambda_{0}:=\min \left\{20(83 \mu+103)^{-1}, \lambda\right\}$. Then for $A \geq \tau_{0}^{-d}, a+\tau^{\lambda_{0}} \leq b^{\prime} \leq b$ we have

$$
\sum_{A^{1 / d}\left(a+\tau^{\lambda}\right)<n \leq A^{1 / d} b^{\prime}} \psi\left(f_{A}(n)\right) \ll A^{46 /(73 d)}(\log A)^{315 / 146}+A^{(\lambda \mu+1) /(2 d)} .
$$

Pr o o f. Let $a<c<b$. For $0<\tau \leq \tau_{0}, a+\tau^{\lambda} \leq x \leq c, k=2,3$, Taylor's theorem gives

$$
\begin{aligned}
\frac{\partial^{k} f}{\partial x^{k}}(\tau, x) & =\frac{\widetilde{f}^{(\mu+2)}(a)}{(\mu+2-k) !}(x-a)^{\mu+2-k}\left(1+O\left(|c-a|+\tau^{1-\lambda(\mu+2-k)}\right)\right), \\
f_{x}(\tau, x) & =\widetilde{f}^{\prime}(a)+\frac{\widetilde{f}^{(\mu+2)}(a)}{(\mu+1) !}(x-a)^{\mu+1}\left(1+O\left(|c-a|+\tau^{1-\lambda(\mu+1)}\right)\right) .
\end{aligned}
$$

Fixing $c$ close to $a$ gives, with $1-\lambda(\mu+1)>0$,

$$
\left|f_{x x}(\tau, x)\right| \asymp|x-a|^{\mu}, \quad\left|f_{x x x}(\tau, x)\right| \asymp|x-a|^{\mu-1} .
$$

In the case $\widetilde{f}^{\prime}(a) \neq 0$ we have $\left|f_{x}(\tau, x)\right| \asymp 1$ and with constants $K_{1}, K_{2}>0$,

$$
\begin{aligned}
\left|f_{x}(\tau, x) f_{x x x}(\tau, x)-3 f_{x x}(\tau, x)^{2}\right| & \geq|x-a|^{\mu-1}\left(K_{1}-K_{2}|x-a|^{\mu+1}\right) \\
& \gg|x-a|^{\mu-1}\left|f_{x}(\tau, x)\right|
\end{aligned}
$$


for $c$ sufficiently close to $a$. In the case $\tilde{f}^{\prime}(a)=0$ we have $\left|f_{x}(\tau, x)\right| \asymp$ $|x-a|^{\mu+1}$ and

$$
\begin{aligned}
& \left|f_{x}(\tau, x) f_{x x x}(\tau, x)-3 f_{x x}(\tau, x)^{2}\right| \\
& \quad=\widetilde{f}^{(\mu+2)}(a)^{2}|x-a|^{2 \mu}\left|\frac{\mu-3(\mu+1)}{(\mu+1) ! \mu !}+O\left(|c-a|+\tau^{1-\lambda(\mu+1)}\right)\right| \\
& \quad \asymp|x-a|^{\mu-1}\left|f_{x}(\tau, x)\right| .
\end{aligned}
$$

For $A^{1 / d}\left(a+\tau^{\lambda}\right) \leq x \leq A^{1 / d} c$ this gives

$$
\begin{gathered}
\left|f_{A}^{\prime \prime}(x)\right| \asymp A^{-1 / d}\left|A^{-1 / d} x-a\right|^{\mu}, \quad\left|f_{A}^{\prime \prime \prime}(x)\right| \asymp A^{-2 / d}\left|A^{-1 / d} x-a\right|^{\mu-1}, \\
\left|f_{A}^{\prime}(x) f_{A}^{\prime \prime \prime}(x)-3 f_{A}^{\prime \prime}(x)^{2}\right| \gg\left|f_{A}^{\prime}(x)\right| A^{-2 / d}\left|A^{-1 / d} x-a\right|^{\mu-1} .
\end{gathered}
$$

Define $M_{0}:=A^{1 / d} \tau^{\lambda_{0}}, M_{J}:=A^{1 / d}(c-a), J:=[\log A], B:=\left(M_{J} / M_{0}\right)^{1 / J}$. Then $B=e^{\lambda_{0} / d}+o(1)$ as $A \rightarrow \infty$. For $1 \leq j \leq J$ define $M_{j}:=M_{0} B^{j}$ and $g_{j}(x):=f_{A}\left(x+\left[2 M_{j-1}-M_{j}\right]+\left[A^{1 / d} a\right]\right) \quad$ on $\left[M_{j}-M_{j-1}, 2\left(M_{j}-M_{j-1}\right)\right]$. Then

$$
\begin{aligned}
S_{1}:= & \sum_{A^{1 / d}\left(a+\tau^{\lambda_{0}}\right)<n \leq A^{1 / d} \min \left\{b^{\prime}, c\right\}} \psi\left(f_{A}(n)\right) \\
& =\sum_{j=1}^{J} \sum_{M_{j}-M_{j-1}<n \leq M_{j}^{\prime}\left(b^{\prime}\right)} \psi\left(g_{j}(n)\right)+O(J)
\end{aligned}
$$

with

$M_{j}\left(b^{\prime}\right):=\min \left\{2\left(M_{j}-M_{j-1}\right), A^{1 / d}\left(b^{\prime}-a\right)-2 M_{j-1}+M_{j}\right\} \leq 2\left(M_{j}-M_{j-1}\right)$.

For $1 \leq j \leq J$ define $T:=A^{-(\mu+1) / d} M_{j-1}^{\mu+3}, M:=M_{j}-M_{j-1}$. For $x \in$ $[M, 2 M]$ it follows that

$$
\begin{gathered}
\left|g_{j}^{\prime \prime}(x)\right| \asymp T M^{-3}, \quad\left|g_{j}^{\prime \prime \prime}(x)\right| \asymp T M^{-4}, \\
\left|g_{j}^{\prime}(x) g_{j}^{\prime \prime \prime}(x)-3 g_{j}^{\prime \prime}(x)^{2}\right| \gg T M^{-4}\left|g_{j}^{\prime}(x)\right| .
\end{gathered}
$$

From the choice of $\lambda_{0}$ it follows that $M T^{-83 / 146} \ll 1$ and $T^{1 / 2} M^{-1}$ $\ll 1$. Theorem 18.2.2 in [2] gives $S_{1} \ll A^{46 /(73 d)}(\log A)^{315 / 146}$. Lemma 4.3 gives

$$
S_{2}:=\sum_{A^{1 / d}} \sum_{c<n \leq A^{1 / d} b^{\prime}} \psi\left(f_{A}(n)\right) \ll A^{46 /(73 d)}(\log A)^{315 / 146} .
$$

By (4.1), $f_{A}^{\prime \prime}$ and $f_{A}^{\prime \prime \prime}$ are zerofree on $\left[A^{1 / d}\left(a+\tau^{\lambda}\right), A^{1 / d}\left(a+\tau^{\lambda_{0}}\right)\right]$ and consequently $f_{A}^{\prime \prime}$ is monotonic. From van der Corput's theorem (Krätzel [3], 
Theorem 2.3) it follows that

$$
\begin{aligned}
S_{3} & :=\sum_{A^{1 / d}\left(a+\tau^{\lambda}\right)<n \leq A^{1 / d}\left(a+\tau^{\lambda_{0}}\right)} \psi\left(f_{A}(n)\right) \\
& \ll \int_{A^{1 / d}\left(a+\tau^{\lambda}\right)} A^{-1 /(3 d)}\left|A^{-1 / d} x-a\right|^{\mu / 3} d x+\left(A^{-1 / d} \tau^{\lambda \mu}\right)^{-1 / 2} \\
& \ll A^{46 /(73 d)}+A^{(1+\lambda \mu) /(2 d)}
\end{aligned}
$$

using the special choice of $\lambda_{0}$.

The main result of this section is

Proposition 4.5. Let $\widetilde{f}^{\prime}(a)$ be an algebraic irrational, $\widetilde{f}^{\prime}, \widetilde{f}^{\prime \prime}, \widetilde{f}^{\prime \prime \prime} z e-$ rofree on $(a, b]$ and $\mu \in \mathbb{N}$ with $\tilde{f}^{(k)}(a)=0$ for $2 \leq k \leq \mu+1$ and $\widetilde{f}^{(\mu+2)}(a) \neq 0$. Let $\delta>0$. Then for $A \geq \tau_{0}^{-d}$ and $a+\tau^{20 /(83 \mu+103)} \leq b^{\prime} \leq b$ we have $\sum_{A^{1 / d} a\left(A^{-1 / d}\right)<n \leq A^{1 / d} b^{\prime}} \psi\left(f_{A}(n)\right) \ll A^{(1 / d)(1-1 / \mu+\delta)}+A^{46 /(73 d)}(\log A)^{315 / 146}$.

Pr o of. Define $\lambda:=1 /(3 \mu+2)$. For $h \in \mathbb{N}$ define

$$
S(h):=\sum_{A^{1 / d}\left(a+\tau^{1 / \mu-\delta}\right)<n \leq A^{1 / d}\left(a+\tau^{\lambda}\right)} e\left(h f_{A}(n)\right) .
$$

From Krätzel [3], Theorem 1.8 for $s=2$ it follows that for arbitrary $H>0$,

$$
\begin{aligned}
S & :=\sum_{A^{1 / d}\left(a+\tau^{1 / \mu-\delta}\right)<n \leq A^{1 / d}\left(a+\tau^{\lambda}\right)} \psi\left(f_{A}(n)\right) \\
& \ll A^{(1-\lambda) / d} H^{-1}+\sum_{h \geq 1} \min \left\{\frac{H^{2}}{h^{3}}, \frac{1}{h}\right\}|S(h)| .
\end{aligned}
$$

Let $\mathcal{C}_{1}$ be the arc of the circle with radius $r:=C A^{1 / d}\left(\tau^{\lambda}-\tau^{1 / \mu-\delta}\right)$ which starts at

$$
P_{1}:=\left(A^{1 / d}\left(a+\tau^{1 / \mu-\delta}\right), f_{A}\left(A^{1 / d}\left(a+\tau^{1 / \mu-\delta}\right)\right)\right)
$$

and proceeds clockwise to its endpoint

$$
P_{2}:=\left(A^{1 / d}\left(a+\tau^{\lambda}\right), f_{A}\left(A^{1 / d}\left(a+\tau^{\lambda}\right)\right)\right)
$$

and whose centre $M$ lies below the line $\left(P_{1} P_{2}\right)$. Here $C \geq 1$ is a constant which is fixed later. The «-constants below are independent of $C$.

Let $2 \alpha$ be the angle under which $\mathcal{C}_{1}$ is seen from its centre $M$ and $\mp \beta$ $(\beta \geq 0)$ the angle between $\left(P_{1} P_{2}\right)$ and the horizontal axis. In the following the upper resp. lower sign is valid whenever $\left(P_{1} P_{2}\right)$ has negative resp. positive slope. From the mean value theorem it follows that

$$
|\mp \tan \beta|=\left|f_{A}^{\prime}(\zeta)\right| \ll 1 \quad \text { with } A^{1 / d}\left(a+\tau^{1 / \mu-\delta}\right)<\zeta<A^{1 / d}\left(a+\tau^{\lambda}\right) .
$$


Consequently, there is a constant $\varepsilon_{0}>0$ with $0 \leq \beta \leq \pi / 2-\varepsilon_{0}$. Therefore

$$
\left|P_{1} P_{2}\right|=(\cos \beta)^{-1}\left(A^{1 / d}\left(a+\tau^{\lambda}\right)-A^{1 / d}\left(a+\tau^{1 / \mu-\delta}\right)\right)<2 r
$$

for sufficiently large $C \geq 1$. For such $C$ the $\operatorname{arc} \mathcal{C}_{1}$ exists in the form described above. From $\sin \alpha=(2 C \cos \beta)^{-1}$ it follows that $\alpha \asymp C^{-1}$. Choose $C \geq 1$ sufficiently large so that $0<\alpha \leq \varepsilon_{0} / 2$. Further conditions on $C$ will be given below. If $m(P)$ denotes the slope of $\mathcal{C}_{1}$ in $P \in \mathcal{C}_{1}$ then

$$
\sup _{P \in \mathcal{C}_{1}}|m(P)| \leq \max \{|\tan (\alpha \mp \beta)|,|\tan (\alpha \pm \beta)|\} \ll 1 .
$$

Let $P_{3} \in \mathcal{C}_{1}$ be the midpoint of $\mathcal{C}_{1}$. For $A^{1 / d}\left(a+\tau^{1 / \mu-\delta}\right) \leq x \leq A^{1 / d}\left(a+\tau^{\lambda}\right)$ there is some $\theta$ between $\zeta$ and $x$ with

$$
\left|f_{A}^{\prime}(x) \pm \tan \beta\right|=\left|f_{A}^{\prime}(x)-f_{A}^{\prime}(\zeta)\right|=\left|f_{A}^{\prime \prime}(\theta)(x-\zeta)\right| \ll \tau^{\lambda} .
$$

For the slope $m_{1}:=\tan (\alpha / 2 \mp \beta)$ of $\left(P_{1} P_{3}\right)$ and $0<\tau \leq \tau_{0}(C)$ it follows that

$$
m_{1}-f_{A}^{\prime}(x)= \pm\left(\cos \theta_{2}\right)^{-2}( \pm \alpha / 2)+O\left(\tau^{\lambda}\right) \geq \alpha / 2+O\left(\tau^{\lambda}\right)>0
$$

with some $\theta_{2}$ between $\beta$ and $\beta \mp \alpha / 2$. Therefore the graph of $f_{A}$ between $P_{1}$ and $P_{2}$ lies below $\left(P_{1} P_{3}\right)$. The same holds for $\left(P_{3} P_{2}\right)$ and consequently the graph of $f_{A}$ lies below $\mathcal{C}_{1}$. Let $F_{A}:\left[A^{1 / d}\left(a+\tau^{1 / \mu-\delta}\right), A^{1 / d}\left(a+\tau^{\lambda}\right)\right] \rightarrow \mathbb{R}$ be the function whose graph is $\mathcal{C}_{1}$. Then we have $\left|F_{A}^{\prime}\right| \ll 1$ by (4.3). For $A^{1 / d}\left(a+\tau^{1 / \mu-\delta}\right) \leq x \leq A^{1 / d}\left(a+\tau^{\lambda}\right)$ we obtain $r^{-1}=\left|F_{A}^{\prime \prime}(x)\right|\left(1+F_{A}^{\prime}(x)^{2}\right)^{-3 / 2}$ and consequently $\left|F_{A}^{\prime \prime}(x)\right| \asymp r^{-1} \asymp C^{-1} A^{-1 / d} \tau^{-\lambda}$. From van der Corput's theorem (Krätzel [3], Theorem 2.1) it follows that, for $h \in \mathbb{N}$,

$$
\stackrel{S_{1}(h)}{:=} \sum_{A^{1 / d}\left(a+\tau^{1 / \mu-\delta}\right)<n \leq A^{1 / d}\left(a+\tau^{\lambda}\right)} e\left(h F_{A}(n)\right) \ll h^{1 / 2} A^{(1-\lambda) /(2 d)} C^{1 / 2} .
$$

The main task is the estimation of $S_{2}(h):=S_{1}(h)-S(h)$. Let $\mathcal{C}_{2}$ be the part of the graph of $f_{A}$ between $P_{1}$ and $P_{2}$ and

$$
\begin{aligned}
B:=\left\{(x, y) \in \mathbb{R}^{2} \mid A^{1 / d}\left(a+\tau^{1 / \mu-\delta}\right) \leq x \leq A^{1 / d}\left(a+\tau^{\lambda}\right),\right. & \\
& \left.f_{A}(x) \leq y \leq F_{A}(x)\right\} .
\end{aligned}
$$

For $\vec{k}:=(k, h) \in \mathbb{Z} \times \mathbb{N}$ define $I(k, h):=\int_{B} e(k x+h y) d x d y$ and the vector field $\vec{\nu}(\vec{x})=\vec{\nu}_{\vec{k}}(\vec{x}):=e(\vec{x} \vec{k}) \vec{k}\left(2 \pi i\|\vec{k}\|^{2}\right)^{-1}$ where $\|\vec{k}\|$ is the Euclidean norm of $\vec{k}$. From Poisson's sum formula (Krätzel [3], p. 23, equation (1.11)),

$$
\begin{aligned}
S_{2}(h) & =\sum_{k \in \mathbb{Z}} \int_{A^{1 / d}\left(a+\tau^{1 / \mu-\delta}\right)}^{A^{1 / d}\left(a+\tau^{\lambda}\right)}\left(e\left(h F_{A}(x)\right)-e\left(h f_{A}(x)\right)\right) e(k x) d x+O(1) \\
& =\sum_{k \in \mathbb{Z}} 2 \pi i h I(k, h)+O(1) .
\end{aligned}
$$


From the divergence theorem it follows that, for $\vec{k} \in \mathbb{Z} \times \mathbb{N}$,

$$
I(\vec{k})=\int_{B} \operatorname{div} \vec{\nu}(\vec{x}) d \vec{x}=-\int_{\mathcal{C}_{1}} \vec{\nu} \cdot \vec{n}^{*} d \sigma+\int_{\mathcal{C}_{2}} \vec{\nu} \cdot \vec{n}^{*} d \sigma
$$

where $\vec{n}^{*}$ is the outer normal unit vector on $\partial B$. The second integral is estimated first. Let $L$ be the arc length of $\tau \mathcal{C}_{2}$ and $\vec{u}:[0, L] \rightarrow \mathbb{R}^{2}$ the natural parametrization of $\tau \mathcal{C}_{2}$. Then $\vec{t}(s)=\vec{u}^{\prime}(s)$ is the tangent unit vector to $\tau \mathcal{C}_{2}$ in $\vec{u}(s)$ and $\vec{u}^{\prime \prime}(s)=\kappa(s) \vec{n}(s)$ where $\kappa(s)$ is the curvature of $\tau \mathcal{C}_{2}$ in $\vec{u}(s)$ and $\vec{n}(s)$ is the normal unit vector to $\tau \mathcal{C}_{2}$ in $\vec{u}(s)$. Let $g(s):=\vec{k} \cdot \vec{u}(s)\|\vec{k}\|^{-1}$. Then

$$
\int_{\mathcal{C}_{2}} \vec{\nu} \cdot \vec{n}^{*} d \sigma=-A^{1 / d}\left(2 \pi i\|\vec{k}\|^{2}\right)^{-1} \int_{0}^{L} e\left(g(s) A^{1 / d}\|\vec{k}\|\right) \vec{k} \cdot \vec{n}(s) d s .
$$

For $0 \leq s \leq L$ we have

$$
\kappa(s) \ll 1 \quad \text { and } \quad\left\|\vec{n}^{\prime}(s)\right\|=\|-\kappa(s) \vec{t}(s)\| \ll 1 .
$$

Furthermore $L \ll \tau^{\lambda}$. Now $\left|g^{\prime}\right|$ is estimated from below.

CASE 1: $\left|g^{\prime}(0)\right| \geq 1 / 2$. For $0 \leq s \leq L$ and $0<\tau \leq \tau_{0}$ it follows from (4.8) and the mean value theorem that $\left|g^{\prime}(s)-g^{\prime}(0)\right|=\left|g^{\prime \prime}(\zeta) s\right| \leq L \kappa(s) \leq 1 / 4$ with $0 \leq \zeta \leq s$. Therefore $\left|g^{\prime}(s)\right| \geq 1 / 4$.

CASE $2:\left|g^{\prime}(0)\right|<1 / 2$. Then $|\vec{k} \cdot \vec{n}(0)| \cdot\|\vec{k}\|^{-1}=\sqrt{1-g^{\prime}(0)^{2}} \geq \sqrt{3 / 4}$ and the mean value theorem and (4.8) give $|\vec{k} \cdot \vec{n}(s)-\vec{k} \cdot \vec{n}(0)| \cdot\|\vec{k}\|^{-1} \leq \sqrt{3} / 4$ for $0 \leq s \leq L$ and $0 \leq \tau \leq \tau_{0}$. Consequently,

$$
|\vec{k} \cdot \vec{n}(s)| \cdot\|\vec{k}\|^{-1} \geq \sqrt{3} / 4 .
$$

Taylor's formula gives for $a+\tau^{1 / \mu-\delta} \leq x \leq a+\tau^{\lambda}$

$$
\begin{aligned}
f_{x x}(\tau, x) & =\frac{\widetilde{f}^{(\mu+2)}(a)}{\mu !}(x-a)^{\mu}+O\left(\tau+|x-a|^{\mu+1}\right) \\
& =\frac{\widetilde{f}^{(\mu+2)}(a)}{\mu !}(x-a)^{\mu}\left(1+O\left(\tau^{\delta \mu}+\tau^{\lambda}\right)\right) \asymp|x-a|^{\mu}
\end{aligned}
$$

and consequently $|\kappa(s)| \asymp\left|f_{x x}\left(\tau, u_{1}(s)\right)\right| \asymp\left|u_{1}(s)-a\right|^{\mu}$. Furthermore

$$
\left|u_{1}(s)-a\right| \asymp \int_{a}^{u_{1}(s)}\left(1+f_{x}(\tau, x)^{2}\right)^{1 / 2} d x=\varrho(\tau)+s
$$

with $\varrho(\tau):=\int_{a}^{a+\tau^{1 / \mu-\delta}}\left(1+f_{x}(\tau, x)^{2}\right)^{1 / 2} d x \geq 0$. With (4.9) it follows that

$$
\left|g^{\prime \prime}(s)\right|=|\vec{k} \cdot \vec{n}(s) \kappa(s)| \cdot\|\vec{k}\|^{-1} \asymp \kappa(s) \asymp(\varrho(\tau)+s)^{\mu} .
$$

In particular $g^{\prime}$ is strictly monotonic and consequently there is exactly one $0 \leq s_{0} \leq L$ with $\left|g^{\prime}\left(s_{0}\right)\right|=\min _{0 \leq s \leq L}\left|g^{\prime}(s)\right|$. Choose $0 \leq \gamma=\gamma_{\vec{k}} \leq \pi / 2$ so that $\pi / 2-\gamma$ is the angle between $\vec{t}(0)$ and $\pm \vec{k}$. 
CASE 2.1: $\left|g^{\prime}\left(s_{0}\right)\right| \geq\left|g^{\prime}(0)\right| / 2$. It follows from $g^{\prime}(0)= \pm \cos (\pi / 2-\gamma)$ that $\left|g^{\prime}\left(s^{\prime}\right)\right| \geq\left|g^{\prime}\left(s_{0}\right)\right| \gg \gamma$ for $0 \leq s^{\prime} \leq L$.

CASE 2.2: $\left|g^{\prime}\left(s_{0}\right)\right|<\left|g^{\prime}(0)\right| / 2$. It follows from (4.10) that for $0 \leq s^{\prime} \leq L$,

$$
\begin{aligned}
\left|g^{\prime}\left(s^{\prime}\right)\right| & \geq\left|g^{\prime}\left(s^{\prime}\right)-g^{\prime}\left(s_{0}\right)\right|=\left|\int_{s_{0}}^{s^{\prime}} g^{\prime \prime}(s) d s\right| \gg\left|\int_{s_{0}}^{s^{\prime}}(\varrho(\tau)+s)^{\mu} d s\right| \\
& \gg\left|\left(\varrho(\tau)+s^{\prime}\right)^{\mu+1}-\left(\varrho(\tau)+s_{0}\right)^{\mu+1}\right| \geq\left|s^{\prime}-s_{0}\right|\left(\varrho(\tau)+s_{0}\right)^{\mu}, \\
\gamma & \ll\left|g^{\prime}(0)\right| \leq 2\left|g^{\prime}(0)-g^{\prime}\left(s_{0}\right)\right| \ll\left|\int_{0}^{s_{0}} g^{\prime \prime}(s) d s\right| \ll\left(\varrho(\tau)+s_{0}\right)^{\mu+1}
\end{aligned}
$$

and consequently $\left|g^{\prime}\left(s^{\prime}\right)\right| \gg\left|s^{\prime}-s_{0}\right| \gamma^{\mu /(\mu+1)}$.

Summarizing, in Case 1 we have $\left|g^{\prime}(s)\right| \geq 1 / 4$ and in Case 2 we have $\left|g^{\prime}(s)\right| \gg \min \left\{\left|s-s_{0}\right| \gamma^{\mu /(\mu+1)}, \gamma\right\}$ for $0 \leq s \leq L$.

Let $\gamma>0$ and $\delta:=\left(A^{1 / d}\|\vec{k}\| \gamma^{\mu /(\mu+1)}\right)^{-1 / 2}$. From (4.7) and (4.8) it follows that in both cases (with arbitrary $s_{0}$ in the first case), after partial integration,

$$
\begin{aligned}
\int_{\mathcal{C}_{2}} \vec{\nu} \cdot \vec{n}^{*} d \sigma & \\
= & -A^{1 / d}\left(2 \pi i\|\vec{k}\|^{2}\right)^{-1}\left(\int_{\left|s-s_{0}\right|<\delta}+\int_{\left|s-s_{0}\right|>\delta}\right) \\
\ll & A^{1 / d}\|\vec{k}\|^{-1} \delta \\
& +\|\vec{k}\|^{-2}\left(\sup _{\left|s-s_{0}\right| \geq \delta}\left|g^{\prime}(s)\right|^{-1}+\int_{\left|s-s_{0}\right| \geq \delta}\left(\left|g^{\prime}(s)\right|^{-1}+\left|g^{\prime \prime}(s)\right| g^{\prime}(s)^{-2}\right) d s\right) .
\end{aligned}
$$

In Case 1 the terms in parentheses are $\ll 1$. In Case $2, g^{\prime \prime}>0$ or $g^{\prime \prime}<0$ by (4.10) and the terms in parentheses are $\ll \delta^{-1} \gamma^{-\mu /(\mu+1)}+\gamma^{-1}$. So in both cases

$$
\int_{\mathcal{C}_{2}} \vec{\nu} \cdot \vec{n}^{*} d \sigma \ll A^{1 /(2 d)}\|\vec{k}\|^{-3 / 2} \gamma^{-\mu /(2(\mu+1))}+\|\vec{k}\|^{-2} \gamma^{-1} .
$$

The same arguments hold for the first integral in (4.6) in a simplified form and therefore only the differences are indicated. The corresponding objects are written with a tilde $\sim$. We have $\widetilde{L} \ll \tau^{\lambda}$ with some $\ll$-constant independent of $C$. Furthermore $\widetilde{\kappa}(s)=A^{1 / d} r^{-1} \asymp C^{-1} \tau^{-\lambda}$ and $\left\|\widetilde{\vec{n}}^{\prime}(s)\right\|=$ $\|-\widetilde{\kappa}(s) \widetilde{\vec{t}}(s)\| \ll C^{-1} \tau^{-\lambda}$ for $0 \leq s \leq \widetilde{L}$.

In the first case $\left|\widetilde{g}^{\prime}(0)\right| \geq 1 / 2$ use $\left|\widetilde{g}^{\prime}(s)-\widetilde{g}^{\prime}(0)\right| \ll C^{-1}$ for $0 \leq s \leq \widetilde{L}$. If we choose $C$ sufficiently large the upper bound becomes $\leq 1 / 4$. In the second case $\left|\widetilde{g}^{\prime}(0)\right|<1 / 2$ use $|\vec{k} \cdot \widetilde{\vec{n}}(s)-\vec{k} \cdot \widetilde{\vec{n}}(0)| \cdot\|\vec{k}\|^{-1} \ll C^{-1}$ for $0 \leq s \leq \widetilde{L}$. For $C$ sufficiently large we have $|\vec{k} \cdot \widetilde{\vec{n}}(s)| \cdot\|\vec{k}\|^{-1} \gg 1$. Then $\left|\widetilde{g}^{\prime \prime}(s)\right| \gg C^{-1} \tau^{-\lambda} \gg 1$ 
for $0<\tau \leq \tau_{0}(C)$. This was the last condition on $C$. Now, $\widetilde{g}^{\prime}$ is strictly monotonic. Let $\left|\widetilde{g}^{\prime}\right|$ take its infimum at $s_{0}$. Then $\left|\widetilde{g}^{\prime}\left(s^{\prime}\right)\right| \geq\left|\widetilde{g}^{\prime}\left(s^{\prime}\right)-\widetilde{g}^{\prime}\left(s_{0}\right)\right|=$ $\left|\int_{s_{0}}^{s^{\prime}} \widetilde{g}^{\prime \prime}(s) d s\right| \gg\left|s^{\prime}-s_{0}\right|$ for $0 \leq s^{\prime} \leq \widetilde{L}$. Similar arguments with $\delta:=$ $\left(A^{1 / d}\|\vec{k}\|\right)^{-1 / 2}$ and $\left|\widetilde{g}^{\prime \prime}(s)\right| \ll C^{-1} \tau^{-\lambda}$ for $0 \leq s \leq \widetilde{L}$ give

$$
\int_{\mathcal{C}_{1}} \vec{\nu} \cdot \vec{n}^{*} d \sigma \ll A^{1 /(2 d)}\|\vec{k}\|^{-3 / 2} .
$$

From (4.11) and (4.6) it follows that

$$
I(\vec{k}) \ll A^{1 /(2 d)}\|\vec{k}\|^{-3 / 2} \gamma_{\vec{k}}^{-\mu /(2(\mu+1))}+\|\vec{k}\|^{-2} \gamma_{\vec{k}}^{-1}
$$

for all $\vec{k}=(k, h) \in \mathbb{Z} \times \mathbb{N}$ with $0<\gamma_{\vec{k}} \leq \pi / 2$ and $\pi / 2-\gamma_{\vec{k}}$ the angle between $\vec{t}(0)$ and $\pm \vec{k}$.

Next $\gamma_{\vec{k}}$ is estimated from below. Set $\left(\tau_{1}, \tau_{2}\right):=\vec{t}(0)$ and for $h \in \mathbb{N}$ define $k(h) \in \mathbb{Z}$ by $-1 / 2<k(h)+h \tau_{2} / \tau_{1} \leq 1 / 2$. From Taylor's theorem it follows that

$$
\begin{aligned}
\frac{\tau_{2}}{\tau_{1}} & =f_{x}\left(\tau, a+\tau^{1 / \mu-\delta}\right) \\
& =\widetilde{f}^{\prime}(a)+\sum_{l=1}^{\mu} \frac{\widetilde{f}^{(l+1)}(a)}{l !} \tau^{(1 / \mu-\delta) l}+O\left(\tau^{(1 / \mu-\delta)(\mu+1)}+\tau\right) \\
& =\widetilde{f}^{\prime}(a)+O(\tau) .
\end{aligned}
$$

In particular $\left|\tau_{2} / \tau_{1}\right| \asymp 1$ and consequently $k(h) \ll h$ for $h \in \mathbb{N}$. Furthermore $1 \geq \tau_{1}=\left(1+f_{x}\left(\tau, a+\tau^{1 / \mu-\delta}\right)^{2}\right)^{-1 / 2} \gg 1$. Applying Roth's theorem to the algebraic irrational $\widetilde{f}^{\prime}(a)$ gives $\left|\tilde{f}^{\prime}(a)+k(h) / h\right| \geq K_{3}(\delta) h^{-(2+\delta)}$ for $h \in \mathbb{N}$ with some constant $K_{3}(\delta)>0$. From (4.13) it follows that with some constant $K_{4}>0$ for $\vec{k}=(k(h), h)$,

$$
\begin{aligned}
\gamma_{\vec{k}} & \geq \sin \gamma_{\vec{k}}=\left|k(h) \tau_{1}+h \tau_{2}\right| \cdot\|\vec{k}\|^{-1} \\
& \gg|| k(h)+h \widetilde{f}^{\prime}(a)|-| h \widetilde{f}^{\prime}(a)-h \tau_{2} / \tau_{1}|| h^{-1} \\
& \geq\left(K_{3} h^{-(1+\delta)}-K_{4} h \tau\right) h^{-1} .
\end{aligned}
$$

For $1 \leq h \leq A^{(1-\delta / 2) /(2 d)}$ we have $h \tau / h^{-(1+\delta)} \ll \tau^{\delta^{2} / 4}$ and consequently $\gamma_{\vec{k}} \gg h^{-(2+\bar{\delta})}$ for sufficiently large $A$. (4.12) gives, for $1 \leq h \leq A^{(1-\delta / 2) /(2 d)}$,

$$
I(k(h), h) \ll A^{1 /(2 d)} h^{(1+\delta / 2) \mu /(\mu+1)-3 / 2}+h^{\delta} .
$$

For $\vec{k}=(k, h) \in \mathbb{Z} \times \mathbb{N}, k \neq k(h)$ the choice of $k(h)$ gives

$$
\begin{aligned}
\gamma_{\vec{k}} & \geq\left|k \tau_{1}+h \tau_{2}\right| \cdot\|\vec{k}\|^{-1} \geq\|\vec{k}\|^{-1} \tau_{1}\left(|k-k(h)|-\left|k(h)+h \tau_{2} / \tau_{1}\right|\right) \\
& \geq\|\vec{k}\|^{-1} \tau_{1}(|k-k(h)|-|k-k(h)| / 2) \gg\|\vec{k}\|^{-1}|k-k(h)| .
\end{aligned}
$$


For $h \in \mathbb{N}$ it follows from (4.12) that

$$
\sum_{k \neq k(h)}|I(k, h)| \ll A^{1 /(2 d)} h^{-1 / 2}+1 .
$$

From (4.5) and (4.14) it follows that $\left|S_{2}(h)\right| \ll A^{1 /(2 d)} h^{1 / 2}$ for $1 \leq h \leq$ $A^{(1-\delta / 2) /(2 d)}$. The trivial estimate $S(h) \ll A^{(1-\lambda) / d}$ gives, with $(4.2)$ and (4.4) for $H \geq 1$,

$$
\begin{aligned}
S & \ll A^{(1-\lambda) / d} H^{-1}+\sum_{h \leq A^{(1-\delta / 2) /(2 d)}} \min \left\{\frac{H^{2}}{h^{3}}, \frac{1}{h}\right\} A^{1 /(2 d)} h^{1 / 2} \\
& +\sum_{h \geq A^{(1-\delta / 2) /(2 d)}} \frac{H^{2}}{h^{3}} A^{(1-\lambda) / d} \\
& \ll A^{(1-\lambda) / d} H^{-1}+A^{1 /(2 d)} H^{1 / 2}+H^{2} A^{(1 / d)(\delta / 2-\lambda)} .
\end{aligned}
$$

The optimal choice $H=A^{2(1 / 2-\lambda) /(3 d)}$ gives $S \ll A^{(2-\lambda) /(3 d)}$. Lemma 4.4 and the choice of $\lambda$ give

$$
\begin{aligned}
\sum_{A^{1 / d} a\left(A^{-1 / d}\right)<n \leq A^{1 / d} b^{\prime}} \psi & \left(f_{A}(n)\right) \\
\ll & A^{1 / d} \tau^{1 / \mu-\delta}+A^{(2-\lambda) /(3 d)} \\
& \quad+A^{46 /(73 d)}(\log A)^{315 / 146}+A^{(\lambda \mu+1) /(2 d)} \\
\ll & A^{(1 / d)(1-1 / \mu+\delta)}+A^{46 /(73 d)}(\log A)^{315 / 146} .
\end{aligned}
$$

5. The case of rational slope. The following lemma is used in the asymptotic evaluation of the lattice integral.

Lemma 5.1. Let $0<\omega<1$. For $v \geq 0,0 \neq T \in \mathbb{R}$, define

$$
H(v, T):=\int_{v}^{\infty} u^{\omega-1} e^{-i T u} d u .
$$

Then for $v>0, T \neq 0$,

$$
|H(v, T)| \ll_{\omega}|T|^{-1} v^{\omega-1}, \quad|H(v, T)| \ll_{\omega}|T|^{-\omega}+v^{\omega}
$$

and for $T \neq 0$,

$$
H(0, T)=\Gamma(\omega) e^{-(\operatorname{sign} T) i \omega \pi / 2}|T|^{-\omega} .
$$

Proof. The first and the third statement can be found in [4], p. 155. The second statement is a slight generalization of the essential part of the proof of Lemma 5 in [4]. Let $T>0, R>v>0$, let $\mathcal{K}_{1}$ resp. $\mathcal{K}_{3}$ be the straight paths from $i v$ to $i R$ resp. from $v$ to $R$, and $\mathcal{K}_{2}$ resp. $\mathcal{K}_{4}$ the circular 
arcs with centre 0 from $i v$ to $v$ resp. from $R$ to $i R$. Cauchy's theorem gives

$$
\int_{\mathcal{K}_{1}} z^{\omega-1} e^{-T z} d z=\int_{\mathcal{K}_{2}}+\int_{\mathcal{K}_{3}}+\int_{\mathcal{K}_{4}} .
$$

Decomposing $\mathcal{K}_{4}$ into those $z$ with $\Re z \geq R^{(1-\omega) / 2}$ and their complement gives $\int_{\mathcal{K}_{4}} \ll R^{\omega} \exp \left(-T R^{(1-\omega) / 2}\right)+R^{(\omega-1) / 2}$. Letting $R \rightarrow \infty$ in (5.3) gives

$$
\begin{aligned}
e^{i \pi \omega / 2} H(v, T) & =\int_{\mathcal{K}_{2}} z^{\omega-1} e^{-T z} d z+\int_{v}^{\infty} z^{\omega-1} e^{-T z} d z \\
& \ll v^{\omega}+\int_{0}^{\infty} t^{\omega-1} e^{-T t} d t \ll \omega v^{\omega}+T^{-\omega} .
\end{aligned}
$$

The case $T<0$ can be reduced to this case by complex conjugation.

The following proposition is the analogue of Proposition 4.5 in the case of rational slope at the point of vanishing curvature. The proof follows the general line of [8].

Proposition 5.2. Assume $0 \leq a<b$ and $\tilde{f}>0$ on $[a, b]$. Let $\widetilde{f}^{\prime}(a)=$ $-p / q, p \in \mathbb{N}_{0}, q \in \mathbb{N},(p, q)=1$, and $\mu \in \mathbb{N}$ with $\widetilde{f}^{(k)}(a)=0$ for $2 \leq k \leq$ $\mu+1$ and $\widetilde{f}^{(\mu+2)}(a) \neq 0$. Let $\widetilde{f}^{\prime}, \widetilde{f}^{\prime \prime}, \widetilde{f}^{\prime \prime \prime}$ be zerofree on $(a, b]$. There is some $\lambda>0$ with the property: For $A \geq \tau_{0}^{-d}, a+\tau^{\lambda} \leq b^{\prime} \leq b$ we have

$$
\begin{aligned}
N:= & \#\left\{(x, y) \in \mathbb{Z}^{2} \mid A^{1 / d} a\left(A^{-1 / d}\right)<x \leq A^{1 / d} b^{\prime}, 0<y \leq f_{A}(x)\right\} \\
= & \int_{A^{1 / d} a\left(A^{-1 / d}\right)}^{A^{1 / d} b^{\prime}} f_{A}(x) d x-\psi\left(A^{1 / d} b^{\prime}\right) f_{A}\left(A^{1 / d} b^{\prime}\right) \\
& +\psi\left(A^{1 / d} a\left(A^{-1 / d}\right)\right) f_{A}\left(A^{1 / d} a\left(A^{-1 / d}\right)\right)+\frac{1}{2} A^{1 / d}\left(a\left(A^{-1 / d}\right)-b^{\prime}\right) \\
& +C A^{(1 / d)(1-1 /(\mu+2))} \\
& \times \sum_{h \geq 1} \frac{1}{h^{1+1 /(\mu+2)}} \sin \left(2 \pi h\left(A^{1 / d}(p a+q \widetilde{f}(a))+q f_{\tau}(0, a)\right)+\frac{\pi(-1)^{\alpha+1}}{2(\mu+2)}\right) \\
& +O\left(A^{(1 / d)\left(1-1 /(\mu+2)-1 /(\mu+2)^{2}\right)} \log A+A^{46 /(73 d)}(\log A)^{315 / 146}\right) .
\end{aligned}
$$

The constants are defined by

$$
\begin{aligned}
\alpha:= & \begin{cases}0, & \widetilde{f}^{(\mu+2)}(a)<0, \\
1, & \widetilde{f}^{(\mu+2)}(a)>0,\end{cases} \\
C:= & q^{-1-1 /(\mu+2)}((\mu+1) !)^{1 /(\mu+2)}(\mu+2)^{-(\mu+1) /(\mu+2)} \Gamma\left(\frac{1}{\mu+2}\right) \\
& \times 2^{-1 /(\mu+2)} \pi^{-1-1 /(\mu+2)}\left|\widetilde{f}^{(\mu+2)}(a)\right|^{-1 /(\mu+2)} .
\end{aligned}
$$


Proof. Choose $\varrho:=(\mu+3)(\mu+2)^{-2}$ and $0<\lambda<1 /(\mu+1)$. In what follows several further conditions will be imposed on $\lambda$. Define $\kappa:=$ $\min \{\lambda, 1-\varrho(\mu+1)\}>0$. Define

$$
G_{\tau}(x):=p x+q f(\tau, x) \quad \text { for } a+\tau^{\varrho} \leq x \leq a+\tau^{\lambda} .
$$

Taylor's formula gives, for these $x$,

$$
\begin{aligned}
f_{x}(\tau, x) & =-\frac{p}{q}+\frac{\widetilde{f}^{(\mu+2)}(a)}{(\mu+1) !}(x-a)^{\mu+1}+O\left(|x-a|^{\mu+2}+\tau\right) \\
& =-\frac{p}{q}+\frac{\widetilde{f}^{(\mu+2)}(a)}{(\mu+1) !}(x-a)^{\mu+1}\left(1+O\left(\tau^{\kappa}\right)\right) .
\end{aligned}
$$

In the following only the case $\widetilde{f}^{(\mu+2)}(a)<0$ is handled. The opposite case is completely analogous. Then $G_{\tau}^{\prime}(x)=p+q f_{x}(\tau, x)<p+q(-p / q)=0$ by (5.5). Define $F_{\tau}:=G_{\tau}^{-1}:\left[\beta_{\tau}, \gamma_{\tau}\right] \rightarrow\left[a+\tau^{\varrho}, a+\tau^{\lambda}\right]$ with $\beta_{\tau}=G_{\tau}\left(a+\tau^{\lambda}\right)$, $\gamma_{\tau}=G_{\tau}\left(a+\tau^{\varrho}\right)$. For $t \in \mathbb{Z}$ define

$$
\begin{array}{r}
N(t):=\#\left\{x \in \mathbb{Z} \mid p x \equiv t(q), A^{1 / d}\left(a+\tau^{\varrho}\right)<x \leq A^{1 / d}\left(a+\tau^{\lambda}\right),\right. \\
\left.p x<t \leq A^{1 / d} G_{\tau}\left(A^{-1 / d} x\right)\right\} .
\end{array}
$$

Then

$$
\begin{aligned}
\#\left\{(x, y) \in \mathbb{Z}^{2} \mid A^{1 / d}\left(a+\tau^{\varrho}\right)<x \leq A^{1 / d}\left(a+\tau^{\lambda}\right), 0<y\right. & \left.\leq f_{A}(x)\right\} \\
& =\sum_{t \in \mathbb{Z}} N(t) .
\end{aligned}
$$

For $t>A^{1 / d} \gamma_{\tau}$ it follows that $N(t)=0$. For $A^{1 / d} \beta_{\tau} \geq t$, $N(t)=\#\left\{(x, y) \in \mathbb{Z}^{2} \mid p x+q y=t, A^{1 / d}\left(a+\tau^{\varrho}\right)<x \leq A^{1 / d}\left(a+\tau^{\lambda}\right), y>0\right\}$ and consequently

$$
\begin{array}{r}
\sum_{t \leq A^{1 / d} \beta_{\tau}} N(t)=\#\left\{(x, y) \in \mathbb{Z}^{2} \mid A^{1 / d}\left(a+\tau^{\varrho}\right)<x \leq A^{1 / d}\left(a+\tau^{\lambda}\right),\right. \\
\left.0<y \leq(1 / q)\left(A^{1 / d} \beta_{\tau}-p x\right)\right\} .
\end{array}
$$

Here $\left(A^{1 / d} \beta_{\tau}-p x\right) / q \geq A^{1 / d} f\left(\tau, a+\tau^{\lambda}\right)>0$ for sufficiently large $A$. Partial summation and $\int_{u}^{v} \psi(x) d x \ll 1$ for $u<v$ give

$$
\begin{aligned}
& \sum_{t \leq A^{1 / d} \beta_{\tau}} N(t) \\
& =\frac{1}{q}\left(A^{1 / d} \beta_{\tau}-\psi\left(A^{1 / d} \beta_{\tau}\right)\right) A^{1 / d}\left(\tau^{\lambda}-\tau^{\varrho}\right)-\frac{p}{2 q} A^{2 / d}\left(a+\tau^{\lambda}\right)^{2} \\
& \quad+\frac{p}{2 q} A^{2 / d}\left(a+\tau^{\varrho}\right)^{2}-\psi\left(A^{1 / d}\left(a+\tau^{\lambda}\right)\right) A^{1 / d} f\left(\tau, a+\tau^{\lambda}\right)+O(1) \\
& \quad+\psi\left(A^{1 / d}\left(a+\tau^{\varrho}\right)\right) \frac{1}{q} A^{1 / d}\left(\beta_{\tau}-p\left(a+\tau^{\varrho}\right)\right)-\frac{1}{2} A^{1 / d}\left(\tau^{\lambda}-\tau^{\varrho}\right) .
\end{aligned}
$$


For $A^{1 / d} \beta_{\tau}<t \leq A^{1 / d} \gamma_{\tau}$ we have

$$
N(t)=\#\left\{x \in \mathbb{Z} \mid p x \equiv t(q), A^{1 / d}\left(a+\tau^{\varrho}\right)<x \leq A^{1 / d} F_{\tau}\left(A^{-1 / d} t\right)\right\}
$$

because $F_{\tau}$ is strictly decreasing and $t-p x \geq A^{1 / d} q f\left(\tau, a+\tau^{\lambda}\right)>0$ for sufficiently large $A$. For $t_{0} \in\{0, \ldots, q-1\}$ there is exactly one $x_{0}=x_{0}\left(t_{0}\right) \in$ $\{0, \ldots, q-1\}$ with $t_{0} \equiv p x_{0}(q)$. Decomposing the range of $t$ into the remainder classes modulo $q$ gives

$$
\sum_{A^{1 / d} \beta_{\tau}<t \leq A^{1 / d} \gamma_{\tau}} N(t)=H+S_{1}+S_{2}
$$

with

$$
H=\frac{1}{q} A^{1 / d} \sum_{t_{0}=0}^{q-1} \sum_{A^{1 / d} \beta_{\tau}<t \leq A^{1 / d} \gamma_{\tau}, t \equiv t_{0}(q)}\left(F_{\tau}\left(A^{-1 / d} t\right)-\left(a+\tau^{\varrho}\right)\right),
$$

$S_{1}=-\sum_{t_{0}=0}^{q-1} \sum_{A^{1 / d} \beta_{\tau}<t \leq A^{1 / d} \gamma_{\tau}, t \equiv t_{0}(q)} \psi\left(\frac{1}{q}\left(A^{1 / d} F_{\tau}\left(A^{-1 / d} t\right)-x_{0}\left(t_{0}\right)\right)\right)$,

5.9) $\quad S_{2}=\frac{1}{q} A^{1 / d}\left(\gamma_{\tau}-\beta_{\tau}\right) \psi\left(A^{1 / d}\left(a+\tau^{\varrho}\right)\right)+O(1)$.

Partial summation and substitution $t=A^{1 / d} G_{\tau}(x)$ give

$$
\begin{aligned}
H= & A^{2 / d} \int_{a+\tau^{\varrho}}^{a+\tau^{\lambda}} f(\tau, x) d x+\frac{1}{q} A^{2 / d} \beta_{\tau}\left(\tau^{\varrho}-\tau^{\lambda}\right)-\frac{p}{2 q} A^{2 / d}\left(a+\tau^{\varrho}\right)^{2} \\
& +\frac{p}{2 q} A^{2 / d}\left(a+\tau^{\lambda}\right)^{2}+\frac{1}{q} A^{1 / d} \psi\left(A^{1 / d} \beta_{\tau}\right)\left(\tau^{\lambda}-\tau^{\varrho}\right) \\
& +\frac{1}{q} \int_{A^{1 / d} \beta_{\tau}}^{A^{1 / d} \gamma_{\tau}} F_{\tau}^{\prime}\left(A^{-1 / d} t\right) \psi(t) d t
\end{aligned}
$$

A trivial estimation of the lattice remainder and $f_{A}^{\prime}(x) \ll 1$ give

$$
\begin{gathered}
\#\left\{(x, y) \in \mathbb{Z}^{2} \mid A^{1 / d} a\left(A^{-1 / d}\right)<x \leq A^{1 / d}\left(a+\tau^{\varrho}\right), 0<y \leq f_{A}(x)\right\} \\
=\int_{A^{1 / d} a\left(A^{-1 / d}\right)}^{A^{1 / d}\left(a+\tau^{\varrho}\right)} f_{A}(x) d x-\psi\left(A^{1 / d}\left(a+\tau^{\varrho}\right)\right) f_{A}\left(A^{1 / d}\left(a+\tau^{\varrho}\right)\right) \\
+\psi\left(A^{1 / d} a\left(A^{-1 / d}\right)\right) f_{A}\left(A^{1 / d} a\left(A^{-1 / d}\right)\right)+O\left(A^{1 / d} \tau^{\varrho}\right) .
\end{gathered}
$$

Choose $0<\lambda \leq 20 /(83 \mu+103)<19 /(73 \mu)$. Partial summation and Lemma 4.4 give

$$
\#\left\{(x, y) \in \mathbb{Z}^{2} \mid A^{1 / d}\left(a+\tau^{\lambda}\right)<x \leq A^{1 / d} b^{\prime}, 0<y \leq f_{A}(x)\right\}
$$




$$
\begin{aligned}
= & \int_{A^{1 / d}\left(a+\tau^{\lambda}\right)}^{A^{1 / d} b^{\prime}} f_{A}(x) d x-\psi\left(A^{1 / d} b^{\prime}\right) f_{A}\left(A^{1 / d} b^{\prime}\right) \\
& +\psi\left(A^{1 / d}\left(a+\tau^{\lambda}\right)\right) f_{A}\left(A^{1 / d}\left(a+\tau^{\lambda}\right)\right)+\int_{A^{1 / d}\left(a+\tau^{\lambda}\right)}^{A^{1 / d} b^{\prime}} f_{A}^{\prime}(x) \psi(x) d x \\
& -\frac{1}{2} A^{1 / d}\left(b^{\prime}-a-\tau^{\lambda}\right)+O\left(A^{46 /(73 d)}(\log A)^{315 / 146}\right) .
\end{aligned}
$$

The function $\psi_{1}(x):=\int_{0}^{x} \psi(t) d t, x \in \mathbb{R}$, is continuous, piecewise continuously differentiable and bounded. From $f_{A}^{\prime} \ll 1, f_{A}^{\prime \prime} \ll A^{-1 / d}$ it follows by partial integration that the second integral above is $\ll 1$. Together with (5.6) to (5.11) it follows that

$$
\begin{aligned}
N= & \int_{A^{1 / d} a(\tau)}^{A^{1 / d} b^{\prime}} f_{A}(x) d x+\frac{1}{q} I+S_{1}-\frac{1}{2} A^{1 / d}\left(b^{\prime}-a(\tau)\right) \\
& +\psi\left(A^{1 / d} a(\tau)\right) f_{A}\left(A^{1 / d} a(\tau)\right)-\psi\left(A^{1 / d} b^{\prime}\right) f_{A}\left(A^{1 / d} b^{\prime}\right) \\
& +O\left(A^{1 / d} \tau^{\varrho}\right)+O\left(A^{46 /(73 d)}(\log A)^{315 / 146}\right)
\end{aligned}
$$

with

$$
I:=\int_{A^{1 / d} \beta_{\tau}}^{A^{1 / d} \gamma_{\tau}} F_{\tau}^{\prime}\left(A^{-1 / d} t\right) \psi(t) d t
$$

The asymptotic development of this integral is done with tools from [4]. The asymptotic behaviour of $F_{\tau}^{\prime \prime}$ and $F_{\tau}^{\prime \prime \prime}$ cannot be determined as in that paper because the influence of $\tau$ and $x$ on the value of $f(\tau, x)$ cannot be separated by inverting functions.

Define $\delta_{\tau}:=p a+q \widetilde{f}(a)+q f_{\tau}(0, a) \tau$. For $a+\tau^{\varrho} \leq x \leq a+\tau^{\lambda}$, Taylor's formula gives

$$
f(\tau, x)
$$

$$
\begin{aligned}
& =\frac{1}{q} \delta_{\tau}-\frac{p}{q} x+\frac{\widetilde{f}^{(\mu+2)}(a)}{(\mu+2) !}(x-a)^{\mu+2}\left(1+O\left(|x-a|+\tau|x-a|^{-\mu-1}\right)\right) \\
& =\frac{1}{q} \delta_{\tau}-\frac{p}{q} x+\frac{\widetilde{f}^{(\mu+2)}(a)}{(\mu+2) !}(x-a)^{\mu+2}\left(1+O\left(\tau^{\kappa}\right)\right) .
\end{aligned}
$$

We have $p a+q \tilde{f}(a)>0$ and consequently $\delta_{\tau}>0$ and $\delta_{\tau} \asymp 1$ for sufficiently large $A$. Now (5.13) gives

$$
\delta_{\tau}-\gamma_{\tau}=-\frac{q \widetilde{f}^{(\mu+2)}(a)}{(\mu+2) !} \tau^{\varrho(\mu+2)}\left(1+O\left(\tau^{1-\varrho(\mu+1)}\right)\right)
$$


and in particular $\gamma_{\tau}<\delta_{\tau}$. From (5.14) it follows analogously that

$$
\delta_{\tau}-\beta_{\tau} \asymp \tau^{\lambda(\mu+2)} .
$$

For $\beta_{\tau} \leq t \leq \gamma_{\tau}$ it follows from (5.14) that

$$
t=G_{\tau}\left(F_{\tau}(t)\right)=\delta_{\tau}+q\left(F_{\tau}(t)-a\right)^{\mu+2} \frac{\widetilde{f}^{(\mu+2)}(a)}{(\mu+2) !}\left(1+O\left(\tau^{\kappa}\right)\right)
$$

and consequently

$$
\left(\delta_{\tau}-t\right)^{1 /(\mu+2)} \asymp F_{\tau}(t)-a .
$$

Define $\Phi_{A}(t):=F_{\tau}^{\prime}(t)\left(\delta_{\tau}-t\right)^{(\mu+1) /(\mu+2)}$ on $\left[\beta_{\tau}, \gamma_{\tau}\right]$. From (5.5) and (5.17) it follows that

$$
\begin{aligned}
\Phi_{A}(t) & =\left(\delta_{\tau}-t\right)^{(\mu+1) /(\mu+2)} G_{\tau}^{\prime}\left(F_{\tau}(t)\right)^{-1} \\
& =\left|\frac{q \widetilde{f}^{(\mu+2)}(a)}{(\mu+2) !}\right|^{(\mu+1) /(\mu+2)}\left(\frac{q \widetilde{f}^{(\mu+2)}(a)}{(\mu+1) !}\right)^{-1}+O\left(\tau^{\kappa}\right)
\end{aligned}
$$

and in particular $\left|\Phi_{A}(t)\right| \asymp 1$. Furthermore

$$
\Phi_{A}^{\prime}(t)=-\Phi_{A}(t) G_{\tau}^{\prime}\left(F_{\tau}(t)\right)^{-2}\left(G_{\tau}^{\prime \prime}\left(F_{\tau}(t)\right)+\frac{\mu+1}{\mu+2}\left(\delta_{\tau}-t\right)^{-1} G_{\tau}^{\prime}\left(F_{\tau}(t)\right)^{2}\right) .
$$

For the expression in parentheses Taylor's formula and (5.4) give

$$
\begin{aligned}
& \frac{q}{\mu !} \widetilde{f}^{(\mu+2)}(a)\left(F_{\tau}(t)-a\right)^{\mu}+O\left(\left|F_{\tau}(t)-a\right|^{\mu+1}\right) \\
& \quad+\left(\delta_{\tau}-t\right)^{-1} q \frac{\widetilde{f}^{(\mu+2)}(a)}{\mu !}\left(F_{\tau}(t)-a\right)^{\mu} \\
& \quad \times\left(\frac{q \widetilde{f}^{(\mu+2)}(a)}{(\mu+2) !}\left(F_{\tau}(t)-a\right)^{\mu+2}+O\left(\tau\left|F_{\tau}(t)-a\right|+\left|F_{\tau}(t)-a\right|^{\mu+3}\right)\right) .
\end{aligned}
$$

Using (5.13) gives the more exact asymptotics

$$
t=\delta_{\tau}+q\left(F_{\tau}(t)-a\right)^{\mu+2}\left(\frac{\widetilde{f}^{(\mu+2)}(a)}{(\mu+2) !}+O\left(\left|F_{\tau}(t)-a\right|+\tau\left|F_{\tau}(t)-a\right|^{-\mu-1}\right)\right) .
$$

With (5.18) this gives, for the expression in parentheses,

$$
\begin{aligned}
\frac{q \widetilde{f}^{(\mu+2)}(a)}{\mu !}\left(F_{\tau}(t)-a\right)^{\mu} & +O\left(\left|F_{\tau}(t)-a\right|^{\mu+1}\right) \\
& +\left(\delta_{\tau}-t\right)^{-1} \frac{q \widetilde{f}^{(\mu+2)}(a)}{\mu !}\left(F_{\tau}(t)-a\right)^{\mu} \\
& \times\left(t-\delta_{\tau}+O\left(\left|F_{\tau}(t)-a\right|^{\mu+3}+\tau\left|F_{\tau}(t)-a\right|\right)\right) \\
\ll & \left|F_{\tau}(t)-a\right|^{\mu+1}+\tau\left|F_{\tau}(t)-a\right|^{-1} .
\end{aligned}
$$


From this together with (5.19) and (5.18) it follows that

$$
\left|\Phi_{A}^{\prime}(t)\right| \ll\left(\delta_{\tau}-t\right)^{-(\mu+1) /(\mu+2)}+\tau\left(\delta_{\tau}-t\right)^{-(2 \mu+3) /(\mu+2)} .
$$

Substituting the Fourier development $\psi(t)=(-1 /(2 \pi i)) \sum_{h \neq 0} h^{-1} e(h t)$, which is valid in $\mathbb{L}^{2}[0,1]$, into $I$ gives $I=(-1 /(2 \pi i)) \sum_{h \neq 0} h^{-1} I_{h}$ with

$$
\begin{gathered}
I_{h}=-A^{1 / d} \delta_{\tau}^{1 /(\mu+2)} e^{i T} \int_{\beta_{\tau} / \delta_{\tau}}^{\gamma_{\tau} / \delta_{\tau}} \Phi_{A}\left(\delta_{\tau} t\right) \frac{\partial H}{\partial v}(1-t, T) d t, \\
T:=2 \pi h A^{1 / d} \delta_{\tau}, \quad \omega:=\frac{1}{\mu+2} .
\end{gathered}
$$

Partial integration together with $\gamma_{\tau}<\delta_{\tau}$ and $\delta_{\tau} \asymp 1$ give

$$
\begin{aligned}
I_{h}= & A^{1 / d} \delta_{\tau}^{1 /(\mu+2)} e^{i T} \Phi_{A}\left(\gamma_{\tau}\right) H\left(1-\gamma_{\tau} / \delta_{\tau}, T\right) \\
& +O\left(A^{1 / d}\left|\Phi_{A}\left(\beta_{\tau}\right) H\left(1-\beta_{\tau} / \delta_{\tau}, T\right)\right|\right) \\
& +O\left(A^{1 / d} \int_{\beta_{\tau} / \delta_{\tau}}^{\gamma_{\tau} / \delta_{\tau}}\left|\Phi_{A}^{\prime}\left(\delta_{\tau} t\right) H(1-t, T)\right| d t\right) .
\end{aligned}
$$

The first half of (5.1), $\left|\Phi_{A}(t)\right| \asymp 1$ and (5.16) show that the first error term is $\ll A^{1 / d}|T|^{-1} \tau^{-\lambda(\mu+1)}$. (5.20), (5.15) and (5.1) show that the second error term is

$$
\begin{aligned}
& \ll A^{1 / d} \int_{\beta_{\tau} / \delta_{\tau}}^{\gamma_{\tau} / \delta_{\tau}}|1-t|^{-(\mu+1) /(\mu+2)}|T|^{-1}|1-t|^{\omega-1} d t \\
& \quad+A^{1 / d} \int_{\beta_{\tau} / \delta_{\tau}}^{\gamma_{\tau} / \delta_{\tau}} \tau|1-t|^{-(2 \mu+3) /(\mu+2)}\left(|T|^{-\omega}+|1-t|^{\omega}\right) d t \\
& \ll|h|^{-1} \tau^{-\mu \varrho}+|T|^{-\omega} \tau^{-(\mu+1) \varrho}+\tau^{-\mu \varrho} .
\end{aligned}
$$

(5.15) gives

$$
\left|H(0, T)-H\left(1-\gamma_{\tau} / \delta_{\tau}, T\right)\right| \ll \int_{0}^{1-\gamma_{\tau} / \delta_{\tau}} u^{\omega-1} d u \ll \tau^{\varrho} .
$$

(5.4) yields

$$
F_{\tau}^{\prime}\left(\gamma_{\tau}\right)=G_{\tau}^{\prime}\left(a+\tau^{\varrho}\right)^{-1}=\left(\frac{q \widetilde{f}^{(\mu+2)}(a)}{(\mu+1) !}\right)^{-1} \tau^{-\varrho(\mu+1)}\left(1+O\left(\tau^{1-\varrho(\mu+1)}\right)\right) .
$$

This together with (5.15) gives

$$
\Phi_{A}\left(\gamma_{\tau}\right)=\left(\frac{q \widetilde{f}^{(\mu+2)}(a)}{(\mu+1) !}\right)^{-1}\left|\frac{q \widetilde{f}^{(\mu+2)}(a)}{(\mu+2) !}\right|^{(\mu+1) /(\mu+2)}\left(1+O\left(\tau^{1-\varrho(\mu+1)}\right)\right) .
$$


Putting everything together and using (5.2) and the choice of $\varrho$ we get

$$
\begin{aligned}
I_{h}= & -A^{(1 / d)(1-1 /(\mu+2))}|h|^{-1 /(\mu+2)} e\left(h A^{1 / d} \delta_{\tau}-\frac{\operatorname{sign} h}{4(\mu+2)}\right) C q \pi \\
& +O\left(A^{(1-\varrho) / d}\right)
\end{aligned}
$$

if $0<\lambda \leq(1-\varrho) /(\mu+1)$.

A better estimation is needed for large $T$. Uniformly in $\beta_{\tau} / \delta_{\tau} \leq t_{0} \leq$ $\gamma_{\tau} / \delta_{\tau}$ it follows from partial integration and (5.20) that

$$
\begin{aligned}
I_{h}= & A^{1 / d} \delta_{\tau}^{1 /(\mu+2)}\left[\frac{e\left(h A^{1 / d} \delta_{\tau} t\right)}{2 \pi i h A^{1 / d} \delta_{\tau}} \Phi_{A}\left(\delta_{\tau} t\right)(1-t)^{-(\mu+1) /(\mu+2)}\right]_{\beta_{\tau} / \delta_{\tau}}^{t_{0}} \\
& -A^{1 / d} \delta_{\tau}^{1 /(\mu+2)} \int_{\beta_{\tau} / \delta_{\tau}}^{t_{0}} \frac{e\left(h A^{1 / d} \delta_{\tau} t\right)}{2 \pi i h A^{1 / d} \delta_{\tau}}\left(\delta_{\tau} \Phi_{A}^{\prime}\left(\delta_{\tau} t\right)(1-t)^{-(\mu+1) /(\mu+2)}\right. \\
& \left.+\Phi_{A}\left(\delta_{\tau} t\right) \frac{\mu+1}{\mu+2}(1-t)^{-(\mu+1) /(\mu+2)-1}\right) d t \\
& +A^{1 / d} \delta_{\tau}^{1 /(\mu+2)} \int_{t_{0}}^{\gamma_{\tau} / \delta_{\tau}} e\left(h A^{1 / d} \delta_{\tau} t\right) \Phi_{A}\left(\delta_{\tau} t\right)(1-t)^{-(\mu+1) /(\mu+2)} d t \\
\ll & |h|^{-1}\left(1-t_{0}\right)^{-(\mu+1) /(\mu+2)} \\
& +|h|^{-1} \int_{\beta_{\tau} / \delta_{\tau}}^{t_{0}}(1-t)^{-(\mu+1) /(\mu+2)-1} d t+A^{1 / d} \int_{t_{0}}^{\gamma_{\tau} / \delta_{\tau}}(1-t)^{-(\mu+1) /(\mu+2)} d t .
\end{aligned}
$$

The first term in the «-estimate only appears if $t_{0}>\beta_{\tau} / \delta_{\tau}$.

In the case $1-|h|^{-1} A^{-1 / d} \leq \beta_{\tau} / \delta_{\tau}$ take $t_{0}:=\beta_{\tau} / \delta_{\tau}$. Then from $1-$ $\beta_{\tau} / \delta_{\tau} \leq|h|^{-1} A^{-1 / d}$ it follows that $I_{h} \ll A^{(1 / d)(1-1 /(\mu+2))}|h|^{-1 /(\mu+2)}$.

In the case $1-|h|^{-1} A^{1 / d} \geq \gamma_{\tau} / \delta_{\tau}$ take $t_{0}:=\gamma_{\tau} / \delta_{\tau}$. Then from $1-\gamma_{\tau} / \delta_{\tau} \geq$ $|h|^{-1} A^{-1 / d}$ the same estimate for $I_{h}$ follows.

In the case $\beta_{\tau} / \delta_{\tau}<1-|h|^{-1} A^{-1 / d}<\gamma_{\tau} / \delta_{\tau}$ take $t_{0}:=1-|h|^{-1} A^{-1 / d}$ and again the same estimate for $I_{h}$ follows.

Together with (5.21) this gives

$$
\begin{aligned}
I= & -\frac{1}{2 \pi i} \sum_{0<|h| \leq A} \frac{1}{h} I_{h}-\frac{1}{2 \pi i} \sum_{|h|>A} \frac{1}{h} I_{h} \\
= & \frac{q C}{2 i} A^{(1 / d)(1-1 /(\mu+2))} \\
& \times \sum_{0<|h| \leq A} \frac{\operatorname{sign} h}{|h|^{1+1 /(\mu+2)}} \exp \left(i \operatorname{sign} h\left(2 \pi|h| A^{1 / d} \delta_{\tau}-\frac{\pi}{2(\mu+2)}\right)\right) \\
& +O\left(A^{(1 / d)(1-\varrho)} \log A\right) .
\end{aligned}
$$


Extending the sum over all integers $\neq 0$ gives the additional error term

$$
\ll A^{(1 / d)(1-1 /(\mu+2))} \sum_{|h|>A} \frac{1}{|h|^{1+1 /(\mu+2)}} \ll A^{(1 / d)(1-1 /(\mu+2))-1 /(\mu+2)}
$$

$\ll A^{(1 / d)(1-\varrho)} \log A$.

Next the lattice remainder is estimated by the discrete Hardy-Littlewood method. Taylor's formula gives, for $a+\tau^{\varrho} \leq x \leq a+\tau^{\lambda}, k=2,3$,

$$
\frac{\partial^{k} f(\tau, x)}{\partial x^{k}}=\frac{\widetilde{f}^{(\mu+2)}(a)}{(\mu+2-k) !}(x-a)^{\mu+2-k}\left(1+O\left(\tau^{\kappa}\right)\right) .
$$

Together with (5.5) it follows that

$$
\begin{aligned}
G_{\tau}^{\prime \prime \prime}(x) G_{\tau}^{\prime}(x)-3 G_{\tau}^{\prime \prime}(x)^{2} & \\
= & \frac{q^{2} \widetilde{f}^{(\mu+2)}(a)^{2}(-2 \mu-3)}{\mu !(\mu+1) !}(x-a)^{2 \mu}\left(1+O\left(\tau^{\kappa}\right)\right) \asymp|x-a|^{2 \mu}
\end{aligned}
$$

and analogously $\left|G_{\tau}^{\prime}(x)\right| \asymp|x-a|^{\mu+1},\left|G_{\tau}^{\prime \prime}(x)\right| \asymp|x-a|^{\mu}$.

(5.18) gives, for $\beta_{\tau} \leq t \leq \gamma_{\tau}$,

$$
\begin{aligned}
\left|F_{\tau}^{\prime \prime}(t)\right| & =\left|-\frac{G_{\tau}^{\prime \prime}\left(F_{\tau}(t)\right)}{G_{\tau}^{\prime}\left(F_{\tau}(t)\right)^{3}}\right| \asymp\left|\delta_{\tau}-t\right|^{-2+1 /(\mu+2)}, \\
\left|F_{\tau}^{\prime \prime \prime}(t)\right| & \asymp\left|\delta_{\tau}-t\right|^{-3+1 /(\mu+2)} .
\end{aligned}
$$

Set $M_{0}:=2 q^{-1} A^{46 /(73 d)}, M_{J}:=q^{-1} A^{1 / d}\left(\gamma_{\tau}-\beta_{\tau}-2 \tau^{27 / 73}\right), J:=[\log A]$, $B:=\left(M_{J} / M_{0}\right)^{1 / J}, M_{j}:=M_{0} B^{j}$ for $0 \leq j \leq J$. Arguments similar to those which led to (5.15) give $\gamma_{\tau}-\beta_{\tau}=K_{10} \tau^{\lambda(\mu+2)}(1+o(1))>2 \tau^{27 / 73}$ with some constant $K_{10}>0$ if $0<\lambda<(27 / 73)(\mu+2)^{-1}$. Consequently, $\log B=$ $(1 / d)(27 / 73-\lambda(\mu+2))+o(1)$ as $A \rightarrow \infty$ and therefore $B=K_{11}+o(1)$ with some constant $K_{11}>1$. For $t_{0} \in\{0, \ldots, q-1\}, 1 \leq j \leq J$, set

$$
\begin{aligned}
h_{t_{0}, j}(x):= & q^{-1} A^{1 / d} F_{\tau}\left(A^{-1 / d} t_{0}+A^{-1 / d} q\left(\left[q^{-1} A^{1 / d} \gamma_{\tau}\right]\right.\right. \\
& \left.\left.-x-\left[2 M_{j-1}-M_{j}\right]\right)\right)-q^{-1} x_{0}\left(t_{0}\right)
\end{aligned}
$$

on $\left[M_{j}-M_{j-1}, 2\left(M_{j}-M_{j-1}\right)\right]$. From (5.15) it follows that, for $M_{j}-M_{j-1} \leq$ $x \leq 2\left(M_{j}-M_{j-1}\right)$,

$$
\begin{aligned}
& \left|\delta_{\tau}-A^{-1 / d} t_{0}-A^{-1 / d} q\left(\left[q^{-1} A^{1 / d} \gamma_{\tau}\right]-x-\left[2 M_{j-1}-M_{j}\right]\right)\right| \\
& \quad=\left|\delta_{\tau}-\gamma_{\tau}+A^{-1 / d} q\left(x+2 M_{j-1}-M_{j}\right)+O(\tau)\right| \asymp A^{-1 / d} M_{j-1} .
\end{aligned}
$$

Choosing $M:=M_{j}-M_{j-1}, T:=A^{(1 / d)(1-1 /(\mu+2))}\left(M_{j}-M_{j-1}\right)^{(\mu+3) /(\mu+2)}$, it follows that $\left|h_{t_{0}, j}^{\prime \prime}(x)\right| \asymp T M^{-3},\left|h_{t_{0}, j}^{\prime \prime \prime}(x)\right| \asymp T M^{-4}$ for $x \in[M, 2 M]$. Furthermore $T^{1 / 3} \leq M \ll T^{1 / 2}$. We have

$$
S_{1}=-\sum_{t_{0}=0}^{q-1} \sum_{j=1}^{J} \sum_{M_{j}-M_{j-1}<n \leq 2\left(M_{j}-M_{j-1}\right)} \psi\left(h_{t_{0}, j}(n)\right)+O\left(A^{46 /(73 d)}+J\right) .
$$


Applying Theorem 18.2.2 of [2] gives $S_{1} \ll A^{46 /(73 d)}(\log A)^{315 / 146}$. From (5.12), (5.22) and (5.23) the proposition follows.

6. The tails of the boundary curve. The result of this section is used in the case where $\widetilde{x}_{\nu}^{\prime}(0)=\widetilde{x}_{\nu}^{\prime \prime \prime}(0)=0$ and $\widetilde{x}_{\nu}^{\prime \prime}(0) \neq 0$. Then it is not possible to apply Propositions 4.5 or 5.2. Instead one goes to the inverse function $\widetilde{y}_{\nu}=\widetilde{x}_{\nu}^{-1}$. This function is not $C^{\infty}$ at the point $\widetilde{\xi}_{\nu}$ but the order of the singularity of $\widetilde{y}_{\nu}^{(k)}$ for $k=2,3$ is small so that no additional main term arises. It is possible to improve this section considerably so that it can be applied generally in the case $j_{0}^{(\nu)} \geq 2$. This would give a slight improvement of the error term in the contribution of the tails of the boundary curve. For this the reader is refered to [9].

Proposition 6.1. Let $\widetilde{x}_{\nu}^{\prime}(0)=0 \neq \widetilde{x}_{\nu}^{\prime \prime}(0)$. There are constants $0<\bar{\xi}$ $<\widetilde{\xi}_{\nu}$ and $K>0$ with the property: For $A_{\nu} \geq K, \tau_{\nu}:=A_{\nu}^{-1 / d_{\nu}}, \bar{\xi} \leq \xi \leq$ $\xi_{\nu}\left(\tau_{\nu}\right)$ we have

$$
\begin{aligned}
R:= & \#\left\{(x, y) \in \mathbb{Z}^{2} \mid A_{\nu}^{1 / d_{\nu}} \xi<x \leq A_{\nu}^{1 / d_{\nu}} \xi_{\nu}\left(\tau_{\nu}\right), 0<y \leq A_{\nu}^{1 / d_{\nu}} y_{\nu}\left(\tau_{\nu}, \tau_{\nu} x\right)\right\} \\
= & A_{\nu}^{2 / d_{\nu}} \int_{\xi}^{\xi_{\nu}\left(\tau_{\nu}\right)} y_{\nu}\left(\tau_{\nu}, \tau_{\nu} x\right) d x-\frac{1}{2} A_{\nu}^{1 / d_{\nu}}\left(\xi_{\nu}\left(\tau_{\nu}\right)-\xi\right) \\
& +\psi\left(A_{\nu}^{1 / d_{\nu}} \xi\right) A_{\nu}^{1 / d_{\nu}} y_{\nu}\left(\tau_{\nu}, \xi\right)+O\left(A_{\nu}^{46 /\left(73 d_{\nu}\right)}\left(\log A_{\nu}\right)^{315 / 146}\right) .
\end{aligned}
$$

Proof. Choose $0<\bar{\xi}<\widetilde{\xi}_{\nu}$ with $\widetilde{y}_{\nu}(\bar{\xi})<\widetilde{\eta}_{\nu} / 2$. A second condition on $\bar{\xi}$ will be given below. For $0<\widetilde{x}_{\nu}\left(2 \widetilde{y}_{\nu}(\bar{\xi})\right) \leq x \leq \widetilde{\xi}_{\nu}$ we have

$$
a_{d_{\nu} 0}^{(\nu)} \widetilde{\xi}_{\nu}^{d_{\nu}}=\widetilde{g}_{\nu}\left(\widetilde{\xi}_{\nu}, 0\right)=1=\widetilde{g}_{\nu}\left(x, \widetilde{y}_{\nu}(x)\right)=\sum_{i+j=d_{\nu}} a_{i j}^{(\nu)} x^{i} \widetilde{y}_{\nu}(x)^{j}
$$

and consequently

$$
\begin{aligned}
\widetilde{\xi}_{\nu}-x & \asymp a_{d_{\nu} 0}^{(\nu)} \widetilde{\xi}_{\nu}^{d_{\nu}}-a_{d_{\nu} 0}^{(\nu)} x^{d_{\nu}} \\
& =\widetilde{y}_{\nu}(x)^{j_{0}^{(\nu)}} \sum_{j=j_{0}^{(\nu)}}^{d_{\nu}} a_{d_{\nu}-j, j}^{(\nu)} x^{d_{\nu}-j} \widetilde{y}_{\nu}(x)^{j-j_{0}^{(\nu)}} \asymp \widetilde{y}_{\nu}(x)^{j_{0}^{(\nu)}} .
\end{aligned}
$$

For $0 \leq y \leq 2 \widetilde{y}_{\nu}(\bar{\xi})<\widetilde{\eta}_{\nu}$ it follows that

$$
\left|\widetilde{\xi}_{\nu}-\widetilde{x}_{\nu}(y)\right| \asymp y^{j_{0}^{(\nu)}} .
$$

For $0 \leq k \leq 3,0 \leq y \leq 2 \widetilde{y}_{\nu}(\bar{\xi})$ Taylor's theorem gives

$$
\widetilde{x}_{\nu}^{(k)}(y)=\sum_{l=0}^{j_{0}^{(\nu)}-k} \frac{1}{l !} \widetilde{x}_{\nu}^{(k+l)}(0) y^{l}+O\left(y^{j_{0}^{(\nu)}-k+1}\right) .
$$

With $\widetilde{x}_{\nu}(0)=\widetilde{\xi}_{\nu}$ and $(6.1)$ the choice $k=0$ gives $\widetilde{x}_{\nu}^{(l)}(0)=0$ for $l=1$, $\ldots, j_{0}^{(\nu)}-1$ and $\widetilde{x}_{\nu}^{\left(j_{0}^{(\nu)}\right)}(0) \neq 0$. From the assumptions it follows that $j_{0}^{(\nu)}=2$. 
Let $K_{20}>0$ be a constant which will be chosen appropriately below. For $1 \leq k \leq 3, K_{20} \tau_{\nu} \leq y \leq 2 \widetilde{y}_{\nu}(\bar{\xi})$ Taylor's theorem gives

$$
\frac{\partial^{k} x_{\nu}}{\partial y^{k}}\left(\tau_{\nu}, y\right)=\left(\left(1-\delta_{3 k}\right) \widetilde{x}_{\nu}^{\prime \prime}(0)+O\left(\tau_{\nu} y^{k-2}+y\right)\right) y^{2-k} .
$$

The error term is

$$
\ll \tau_{\nu}\left(1+\left(K_{20} \tau_{\nu}\right)^{-1}\right)+\widetilde{y}_{\nu}(\bar{\xi}) \ll \tau_{\nu}+K_{20}^{-1}+\widetilde{y}_{\nu}(\bar{\xi}) .
$$

Choosing $K_{20}$ sufficiently large and $\bar{\xi}$ sufficiently close to $\widetilde{\xi}_{\nu}$ gives, for $k=$ 1,2 ,

$$
\left|\frac{\partial^{k} x_{\nu}}{\partial y^{k}}\left(\tau_{\nu}, y\right)\right| \asymp y^{2-k}
$$

and

$$
\begin{aligned}
& \left|x_{\nu y y y}\left(\tau_{\nu}, y\right) x_{\nu y}\left(\tau_{\nu}, y\right)-3 x_{\nu y y}\left(\tau_{\nu}, y\right)^{2}\right| \\
& =\left|-3 \widetilde{x}_{\nu}^{\prime \prime}(0)^{2}+O\left(K_{20}^{-1}+\tau_{\nu}+\widetilde{y}_{\nu}(\bar{\xi})\right)\right| \asymp 1 .
\end{aligned}
$$

For $\widetilde{x}_{\nu}\left(2 \widetilde{y}_{\nu}(\bar{\xi})\right) \leq x \leq \widetilde{\xi}_{\nu}-K_{21} \tau_{\nu}\left(\leq \xi_{\nu}\left(\tau_{\nu}\right)\right)$ with sufficiently large $K_{21}>0$ if follows that $2 \widetilde{y}_{\nu}(\bar{\xi}) \geq \widetilde{y}_{\nu}(x) \geq y_{\nu}\left(\tau_{\nu}, x\right) \geq 0$, and (6.1) yields

$$
y_{\nu}\left(\tau_{\nu}, x\right)^{2} \asymp\left|\widetilde{\xi}_{\nu}-x_{\nu}\left(\tau_{\nu}, y_{\nu}\left(\tau_{\nu}, x\right)\right)+O\left(\tau_{\nu}\right)\right| \asymp\left|\widetilde{\xi}_{\nu}-x\right| .
$$

In particular

$$
y_{\nu}\left(\tau_{\nu}, x\right) \gg\left|\widetilde{\xi}_{\nu}-x\right|^{1 / 2} \gg\left(K_{21} \tau_{\nu}\right)^{1 / 2} \geq K_{20} \tau_{\nu}
$$

and from (6.2) it follows that

$$
\begin{aligned}
\left|y_{\nu x}\left(\tau_{\nu}, x\right)\right|= & \left|x_{\nu y}\left(\tau_{\nu}, y_{\nu}\left(\tau_{\nu}, x\right)\right)^{-1}\right| \asymp\left|\widetilde{\xi}_{\nu}-x\right|^{-1 / 2}, \\
\left|y_{\nu x x}\left(\tau_{\nu}, x\right)\right|= & \left|-x_{\nu y y}\left(\tau_{\nu}, y_{\nu}\left(\tau_{\nu}, x\right)\right) x_{\nu y}\left(\tau_{\nu}, y_{\nu}\left(\tau_{\nu}, x\right)\right)^{-3}\right| \asymp\left|\widetilde{\xi}_{\nu}-x\right|^{-3 / 2}, \\
\left|y_{\nu x x x}\left(\tau_{\nu}, x\right)\right|= & \mid-\left(x_{\nu y y y}\left(\tau_{\nu}, y_{\nu}\left(\tau_{\nu}, x\right)\right) x_{\nu y}\left(\tau_{\nu}, y_{\nu}\left(\tau_{\nu}, x\right)\right)\right. \\
& \left.-3 x_{\nu y y}\left(\tau_{\nu}, y_{\nu}\left(\tau_{\nu}, x\right)\right)^{2}\right) x_{\nu y}\left(\tau_{\nu}, y_{\nu}\left(\tau_{\nu}, x\right)\right)^{-5}|\asymp| \widetilde{\xi}_{\nu}-\left.x\right|^{-5 / 2} .
\end{aligned}
$$

Partial summation gives, for $\bar{\xi} \leq \xi \leq \xi_{\nu}\left(\tau_{\nu}\right)$,

$$
\begin{aligned}
R= & \int_{A_{\nu}^{1 / d_{\nu}} \xi}^{A_{\nu}^{1 / d_{\nu}} \xi_{\nu}\left(\tau_{\nu}\right)}\left(A_{\nu}^{1 / d_{\nu}} y_{\nu}\left(\tau_{\nu}, \tau_{\nu} x\right)-\frac{1}{2}\right) d x \\
& +\psi\left(A_{\nu}^{1 / d_{\nu}} \xi\right) A_{\nu}^{1 / d_{\nu}} y_{\nu}\left(\tau_{\nu}, \xi\right) \\
& +O(1)+\int_{A_{\nu}^{1 / d_{\nu}} \xi_{\nu}\left(\tau_{\nu}\right)}^{A_{\nu}^{1 / d_{\nu}} \xi} y_{\nu x}\left(\tau_{\nu}, \tau_{\nu} x\right) \psi(x) d x \\
& -\sum_{A_{\nu}^{1 / d_{\nu}}} \psi\left(A_{\nu}^{1 / d_{\nu}} y_{\nu}\left(\tau_{\nu}^{1 / d_{\nu}}, \tau_{\nu} x\right)\right) .
\end{aligned}
$$


As in the proof of Proposition 5.2 it follows by partial integration that the second integral in $(6.3)$ is

$$
\begin{aligned}
& =\left.y_{\nu x}\left(\tau_{\nu}, \tau_{\nu} x\right) \psi_{1}(x)\right|_{A_{\nu}^{1 / d_{\nu}} \xi} ^{A_{\nu}^{1 / d_{\nu}}\left(\widetilde{\xi}_{\nu}-K_{21} \tau_{\nu}\right)} \\
& A_{\nu}^{1 / d_{\nu}}\left(\widetilde{\xi}_{\nu}-K_{21} \tau_{\nu}\right) \\
& -\int_{A_{\nu}^{1 / d_{\nu}} \xi} \tau_{\nu} y_{\nu x x}\left(\tau_{\nu}, \tau_{\nu} x\right) \psi_{1}(x) d x \\
& A_{\nu}^{1 / d_{\nu}} \xi_{\nu}\left(\tau_{\nu}\right) \\
& +\quad \int y_{\nu x}\left(\tau_{\nu}, \tau_{\nu} x\right) \psi(x) d x \\
& A_{\nu}^{1 / d_{\nu}}\left(\widetilde{\xi}_{\nu}-K_{21} \tau_{\nu}\right) \\
& A_{\nu}^{1 / d_{\nu}}\left(\widetilde{\xi}_{\nu}-K_{21} \tau_{\nu}\right) \\
& \ll\left(K_{21} \tau_{\nu}\right)^{-1 / 2}+\tau_{\nu} \quad \int_{A_{\nu}^{1 / d_{\nu}} \xi}\left|\widetilde{\xi}_{\nu}-\tau_{\nu} x\right|^{-3 / 2} d x \\
& A_{\nu}^{1 / d_{\nu}} \xi_{\nu}\left(\tau_{\nu}\right) \\
& +\quad \int^{-\tilde{\xi}_{\nu}}-\left.\tau_{\nu} x\right|^{-1 / 2} d x \\
& A_{\nu}^{1 / d_{\nu}}\left(\widetilde{\xi}_{\nu}-K_{21} \tau_{\nu}\right)
\end{aligned}
$$

Let $J:=[\log A], M_{0}:=A_{\nu}^{46 /\left(73 d_{\nu}\right)}, M_{J}:=\left[A_{\nu}^{1 / d_{\nu}}\left(\widetilde{\xi}_{\nu}-\bar{\xi}\right)\right], B:=\left(M_{J} M_{0}^{-1}\right)^{1 / J}$, $M_{j}:=M_{0} B^{j}$ for $0 \leq j \leq J$. Then $B=B_{0}+o(1)$ with some constant $B_{0}>1$. For $x \in\left[M_{j}-M_{j-1}, 2\left(M_{j}-M_{j-1}\right)\right]$ define

$$
f_{j}(x):=A_{\nu}^{1 / d_{\nu}} y_{\nu}\left(\tau_{\nu}, \tau_{\nu}\left(\left[A_{\nu}^{1 / d_{\nu}} \widetilde{\xi}_{\nu}\right]-\left[2 M_{j-1}-M_{j}\right]-x\right)\right) .
$$

Then

$$
\begin{aligned}
\left|f_{j}^{\prime \prime}(x)\right| & \asymp A_{\nu}^{-1 / d_{\nu}}\left|\widetilde{\xi}_{\nu}-A_{\nu}^{-1 / d_{\nu}}\left(A_{\nu}^{1 / d_{\nu}} \widetilde{\xi}_{\nu}-2 M_{j-1}+M_{j}-x+O(1)\right)\right|^{-3 / 2} \\
& \asymp A_{\nu}^{-1 / d_{\nu}}\left(A_{\nu}^{-1 / d_{\nu}} M_{j-1}\right)^{-3 / 2} \asymp T\left(M_{j}-M_{j-1}\right)^{-3}, \\
\left|f_{j}^{\prime \prime \prime}(x)\right| & \asymp T\left(M_{j}-M_{j-1}\right)^{-4}
\end{aligned}
$$

with $T:=A_{\nu}^{1 /\left(2 d_{\nu}\right)} M_{j-1}^{3 / 2}$. Furthermore $T^{63 / 146}(\log T)^{63 / 292} \leq\left(M_{j}-M_{j-1}\right)$ $\ll T^{1 / 2}$. From Theorem 18.2.2 of [2] it follows that the lattice remainder in (6.3) is

$$
\begin{aligned}
= & \left.\sum_{j=1}^{J} \sum_{M_{j}-M_{j-1} \leq n \leq \min \left\{2\left(M_{j}-M_{j-1}\right), A_{\nu}^{1 / d_{\nu}}\right.} \psi\left(\widetilde{\xi}_{\nu}-\xi\right)-2 M_{j-1}+M_{j}\right\} \\
& +O\left(A_{\nu}^{46 /\left(73 d_{\nu}\right)}\right) \\
& \ll A_{\nu}^{46 /\left(73 d_{\nu}\right)}\left(\log A_{\nu}\right)^{315 / 146} .
\end{aligned}
$$


7. Combinatorial composition of the results. $R^{\dagger}$ is handled first. Choose $Z \subseteq \mathbb{R}_{0}^{+},|Z|<\infty$, with the properties:

$\left(\mathrm{Z}^{\dagger} 1\right) \quad$ The zeros of $\widetilde{y}_{j}^{\prime \prime} \widetilde{y}_{j}^{\prime \prime \prime}$ in $\left[0, \widetilde{\xi}_{j}\right]$ are contained in $Z$ for $j=1,2$.

$\left(\mathrm{Z}^{\dagger} 2\right) \quad$ The zeros of $\widetilde{x}_{j}^{\prime \prime} \widetilde{x}_{j}^{\prime \prime \prime}$ in $\left[0, \widetilde{\eta}_{j}\right]$ are contained in $\widetilde{y}_{j}(Z)$ for $j=1,2$.

$\left(\mathrm{Z}^{\dagger} 3\right) \quad \xi_{0}, \ldots, \xi_{r}, x_{0}, x_{2} \in Z$.

$\left(\mathrm{Z}^{\dagger} 4\right) \quad$ If $\widetilde{y}_{j}^{\prime}(0)=0 \neq \widetilde{y}_{j}^{\prime \prime}(0)$ for $j=1$ or $j=2$ then the value $\bar{\eta}$ which comes from the application of Proposition 6.1 with $x$ and $y$ interchanged is contained in $\widetilde{y}_{j}(Z)$.

Choose $0=\zeta_{0}<\ldots<\zeta_{n}$ with the properties:

$\left(\mathrm{Z}^{\dagger} 5\right) \quad Z \subseteq\left\{\zeta_{0}, \ldots, \zeta_{n}\right\}$.

( $\left.\mathrm{Z}^{\dagger} 6\right)$ For each $\zeta, \zeta^{\prime} \in Z$ with $\zeta<\zeta^{\prime}$ there is some $1 \leq \nu \leq n$ with $\zeta<\zeta_{\nu}<\zeta^{\prime}$.

The next two lemmas combine the results of Sections 4 to 6 .

Lemma 7.1. Let $j \in\{1,2\}$ and $1 \leq \nu \leq n$ with $\zeta_{\nu}<\widetilde{\xi}_{j}$. There are constants $\kappa, K>0$ so that for $\zeta \in\left(\zeta_{\nu-1}+\tau_{j}^{\kappa}, \zeta_{\nu}-\tau_{j}^{\kappa}\right)$ and $A_{j} \geq K$,

$$
\begin{aligned}
& R:= \#\left\{(x, y) \in \mathbb{Z}^{2} \mid A_{j}^{1 / d_{j}} \zeta_{\nu-1}<x \leq A_{j}^{1 / d_{j}} \zeta,\right. \\
&\left.0<y \leq A_{j}^{1 / d_{j}} y_{j}\left(\tau_{j}, \tau_{j} x\right)\right\} \\
&=A_{j}^{2 / d_{j}} \int_{\zeta_{\nu-1}}^{\zeta} y_{j}\left(\tau_{j}, x\right) d x-\frac{1}{2} A_{j}^{1 / d_{j}}\left(\zeta-\zeta_{\nu-1}\right)+T\left(A_{j}\right)+U\left(A_{j}\right) \\
&+\psi\left(A_{j}^{1 / d_{j}} \zeta_{\nu-1}\right) A_{j}^{1 / d_{j}} y_{j}\left(\tau_{j}, \zeta_{\nu-1}\right)-\psi\left(A_{j}^{1 / d_{j}} \zeta\right) A_{j}^{1 / d_{j}} y_{j}\left(\tau_{j}, \zeta\right) \\
&+O\left(A_{j}^{46 /\left(73 d_{j}\right)}\left(\log A_{j}\right)^{315 / 146}\right) .
\end{aligned}
$$

The representation

$$
\begin{aligned}
T\left(A_{j}\right)= & A_{j}^{\left(1 / d_{j}\right)(1-1 /(\mu+2))} H\left(A_{j}^{1 / d_{j}}\right) \\
& +O\left(A_{j}^{\left(1 / d_{j}\right)\left(1-1 /(\mu+2)-1 /(\mu+2)^{2}\right)} \log A_{j}\right)
\end{aligned}
$$

holds with some constant $\mu \in \mathbb{N}$ and some periodic function $H$ which is given by an absolutely convergent Fourier series. $T\left(A_{j}\right)$ can only occur if $\widetilde{y}_{j}^{\prime \prime}$ has a zero of order $\mu$ at $\zeta_{\nu-1}$ and $\widetilde{y}_{j}^{\prime}\left(\zeta_{\nu-1}\right) \in \mathbb{Q}$.

Furthermore

$$
U\left(A_{j}\right)=O_{\delta}\left(A_{j}^{\left(1 / d_{j}\right)(1-1 / \mu+\delta)}\right)
$$

for each $\delta>0$ and this function can only occur if $\widetilde{y}_{j}^{\prime \prime}$ has a zero of order $\mu$ at $\zeta_{\nu-1}$ and $\widetilde{y}_{j}^{\prime}\left(\zeta_{\nu-1}\right) \notin \mathbb{Q}$.

Proof. From the choice of the $\zeta_{\nu}$ it follows that $\widetilde{y}_{j}^{\prime \prime} \widetilde{y}_{j}^{\prime \prime \prime}$ has zeros in $\left[\zeta_{\nu-1}, \zeta_{\nu}\right]$ at most at $\zeta_{\nu-1}$ or $\zeta_{\nu}$ but not at both points. 
CASE 1: $\widetilde{y}_{j}^{\prime \prime} \widetilde{y}_{j}^{\prime \prime \prime}\left(\zeta_{\nu-1}\right) \neq 0$ and $\widetilde{y}_{j}^{\prime \prime} \widetilde{y}_{j}^{\prime \prime \prime}\left(\zeta_{\nu}\right) \neq 0$. Partial summation gives

$$
\begin{aligned}
R= & \int_{A_{j}^{1 / d_{j}} \zeta_{\nu-1}}^{A_{j}^{1 / d_{j}} \zeta}\left(A_{j}^{1 / d_{j}} y_{j}\left(\tau_{j}, \tau_{j} x\right)-\frac{1}{2}\right) d x \\
& +\int_{A_{j}^{1 / d_{j}} \zeta_{\nu-1}}^{A_{j}^{1 / d_{j}} \zeta} y_{j x}\left(\tau_{j}, \tau_{j} x\right) \psi(x) d x \\
& +\psi\left(A_{j}^{1 / d_{j}} \zeta_{\nu-1}\right)\left(A_{j}^{1 / d_{j}} y_{j}\left(\tau_{j}, \zeta_{\nu-1}\right)-\frac{1}{2}\right) \\
& -\psi\left(A_{j}^{1 / d_{j}} \zeta\right)\left(A_{j}^{1 / d_{j}} y_{j}\left(\tau_{j}, \zeta\right)-\frac{1}{2}\right) \\
& -\sum_{A_{j}^{1 / d_{j}} \zeta_{\nu-1}<x \leq A_{j}^{1 / d_{j}} \zeta} \psi\left(A_{j}^{1 / d_{j}} y_{j}\left(\tau_{j}, \tau_{j} x\right)\right) .
\end{aligned}
$$

The lattice remainder is $O\left(A_{j}^{46 /\left(73 d_{j}\right)}\left(\log A_{j}\right)^{315 / 146}\right)$ as follows from Lemma 4.3. Partial integration gives the bound $O(1)$ for the second integral.

CASE 2: $\widetilde{y}_{j}^{\prime \prime}$ has at $\zeta_{\nu}$ a zero of order $\mu \in \mathbb{N}$. Then $\zeta_{\nu}, 0 \in Z$ and by $\left(\mathrm{Z}^{\dagger} 6\right)$ it follows $\zeta_{\nu-1} \notin Z$, i.e. $\nu \geq 2$. Therefore $\widetilde{y}_{j}^{\prime}(x) \neq 0$ for $x \in\left[\zeta_{\nu-1}, \zeta_{\nu}\right]$. Choose $0<\kappa<\lambda_{0}:=20 /(83 \mu+103)$. By Lemma 4.4 the lattice remainder in (7.4) is

$$
\begin{aligned}
& =\sum_{A_{j}^{1 / d_{j}}\left(-\zeta_{\nu}+\tau_{j}^{\lambda_{0}}\right)<x \leq-A_{j}^{1 / d_{j}} \zeta_{\nu-1}} \psi\left(A_{j}^{1 / d_{j}} y_{j}\left(\tau_{j},-\tau_{j} x\right)\right) \\
& \quad-\sum_{A_{j}^{1 / d_{j}}\left(-\zeta_{\nu}+\tau_{j}^{\lambda_{0}}\right)<x \leq-A_{j}^{1 / d_{j}} \zeta} \psi\left(A_{j}^{1 / d_{j}} y_{j}\left(\tau_{j},-\tau_{j} x\right)\right)+O(1) \\
& \ll A_{j}^{46 /\left(73 d_{j}\right)}\left(\log A_{j}\right)^{315 / 146} .
\end{aligned}
$$

CASE 3: $\widetilde{y}_{j}^{\prime \prime}$ has at $\zeta_{\nu-1}$ a zero of order $\mu \in \mathbb{N}$.

Case 3.1: $\widetilde{y}_{j}^{\prime}\left(\zeta_{\nu-1}\right) \in \mathbb{Q}$. Apply Proposition 5.2. Choose $\kappa>0$ smaller than the value $\lambda$ which is given by this proposition.

CASE 3.2: $\widetilde{y}_{j}^{\prime}\left(\zeta_{\nu-1}\right) \notin \mathbb{Q}$. Then $\widetilde{y}_{j}^{\prime}\left(\zeta_{\nu-1}\right)$ is algebraic over $\mathbb{Q}$ by Lemma 4.2. Apply Proposition 4.5 to the lattice remainder in (7.4) and choose $0<$ $\kappa<20 /(83 \mu+103)$.

CASE 4: There is some $\nu^{\prime} \in\{\nu-1, \nu\}$ with $\widetilde{y}_{j}^{\prime \prime \prime}\left(\zeta_{\nu^{\prime}}\right)=0$ and $\widetilde{y}_{j}^{\prime \prime}\left(\zeta_{\nu^{\prime}}\right) \neq 0$. We have

$$
\begin{aligned}
& R=\#\{(x, y) \in \mathbb{Z}^{2} \mid A_{j}^{1 / d_{j}} y_{j}\left(\tau_{j}, \zeta\right)<y \leq A_{j}^{1 / d_{j}} y_{j}\left(\tau_{j}, \zeta_{\nu-1}\right) \\
&\left.0<x \leq A_{j}^{1 / d_{j}} x_{j}\left(\tau_{j}, \tau_{j} y\right)\right\} \\
&+\left[A_{j}^{1 / d_{j}} \zeta\right]\left[A_{j}^{1 / d_{j}} y_{j}\left(\tau_{j}, \zeta\right)\right]-\left[A_{j}^{1 / d_{j}} \zeta_{\nu-1}\right]\left[A_{j}^{1 / d_{j}} y_{j}\left(\tau_{j}, \zeta_{\nu-1}\right)\right]
\end{aligned}
$$


$\widetilde{x}_{j}^{\prime \prime} \widetilde{x}_{j}^{\prime \prime \prime}$ has no zeros in $\left(\widetilde{y}_{j}\left(\zeta_{\nu}\right), \widetilde{y}_{j}\left(\zeta_{\nu-1}\right)\right)$. On $\left[\widetilde{y}_{j}\left(\zeta_{\nu}\right), \widetilde{y}_{j}\left(\zeta_{\nu-1}\right)\right)$ we have

$$
\begin{gathered}
\widetilde{x}_{j}^{\prime}(y)=\widetilde{y}_{j}^{\prime}\left(\widetilde{x}_{j}(y)\right)^{-1}, \quad \widetilde{x}_{j}^{\prime \prime}(y)=-\widetilde{y}_{j}^{\prime}\left(\widetilde{x}_{j}(y)\right)^{-3} \widetilde{y}_{j}^{\prime \prime}\left(\widetilde{x}_{j}(y)\right), \\
\widetilde{x}_{j}^{\prime \prime \prime}(y)=-\widetilde{y}_{j}^{\prime}\left(\widetilde{x}_{j}(y)\right)^{-5}\left(\widetilde{y}_{j}^{\prime \prime \prime}\left(\widetilde{x}_{j}(y)\right) \widetilde{y}_{j}^{\prime}\left(\widetilde{x}_{j}(y)\right)-3 \widetilde{y}_{j}^{\prime \prime}\left(\widetilde{x}_{j}(y)\right)^{2}\right) .
\end{gathered}
$$

CASE 4.1: $\nu \geq 2$. Then $0<\zeta_{\nu-1}<\zeta_{\nu}<\widetilde{\xi}_{j}$ and (7.6) is valid also in $\widetilde{y}_{j}\left(\zeta_{\nu-1}\right)$. From (7.6) and the assumption of Case 4 it follows that $\widetilde{x}_{j}^{\prime \prime} \widetilde{x}_{j}^{\prime \prime \prime}\left(\widetilde{y}_{j}\left(\zeta_{\nu^{\prime}}\right)\right) \neq 0$. If $\widetilde{x}_{j}^{\prime \prime} \widetilde{x}_{j}^{\prime \prime \prime}$ had a zero in $\left[\widetilde{y}_{j}\left(\zeta_{\nu}\right), \widetilde{y}_{j}\left(\zeta_{\nu-1}\right)\right]$ then this zero would be of the form $\widetilde{y}_{j}(\zeta), \zeta \in Z$. Then $\zeta_{\nu-1} \leq \zeta \leq \zeta_{\nu}, \zeta \neq \zeta_{\nu^{\prime}}, \zeta, \zeta_{\nu^{\prime}} \in Z$. Then there would be some $\zeta_{\nu^{\prime \prime}}$ between $\zeta$ and $\zeta_{\nu^{\prime}}$ and consequently between $\zeta_{\nu-1}$ and $\zeta_{\nu}$, which is a contradiction. Therefore $\widetilde{x}_{j}^{\prime \prime} \widetilde{x}_{j}^{\prime \prime \prime}$ has no zeros in $\left[\widetilde{y}_{j}\left(\zeta_{\nu}\right), \widetilde{y}_{j}\left(\zeta_{\nu-1}\right)\right]$. Applying partial summation and Lemma 4.3 to (7.5) gives

$$
\begin{aligned}
R= & A_{j}^{2 / d_{j}} \int_{\zeta_{\nu-1}}^{\zeta} y_{j}\left(\tau_{j}, x\right) d x-\psi\left(A_{j}^{1 / d_{j}} \zeta\right) A_{j}^{1 / d_{j}} y_{j}\left(\tau_{j}, \zeta\right) \\
& +\psi\left(A_{j}^{1 / d_{j}} \zeta_{\nu-1}\right) A_{j}^{1 / d_{j}} y_{j}\left(\tau_{j}, \zeta_{\nu-1}\right)-\frac{1}{2} A_{j}^{1 / d_{j}}\left(\zeta-\zeta_{\nu-1}\right) \\
& +O\left(A_{j}^{46 /\left(73 d_{j}\right)}\left(\log A_{j}\right)^{315 / 146}\right) .
\end{aligned}
$$

CASE 4.2: $\nu=1, \widetilde{y}_{j}^{\prime}(0) \neq 0$. Then $\zeta_{\nu-1}=\zeta_{0}=0$ and

$$
0 \neq-\widetilde{y}_{j}^{\prime}(0) \widetilde{g}_{j y}\left(0, \widetilde{y}_{j}(0)\right)=\widetilde{g}_{j x}\left(0, \widetilde{y}_{j}(0)\right)=a_{1, d_{1}-1}^{(j)} \widetilde{\eta}_{j}^{d_{j}-1}
$$

and consequently $i_{0}^{(j)}=1$ and $\widetilde{x}_{j} \in C^{\infty}\left[0, \widetilde{\eta}_{j}\right]$. The proof is exactly the same as in Case 4.1.

CASE 4.3: $\nu=1, \widetilde{y}_{j}^{\prime}(0)=0$. Now the argument of Cases 4.1 and 4.2 is no longer valid because $\widetilde{x}_{j}$ is not $C^{\infty}$ at $\widetilde{\eta}_{j}$. We have $\widetilde{y}_{j}^{\prime \prime}(0) \neq 0$ because otherwise $\nu^{\prime}=1$ and $\widetilde{y}_{j}^{\prime \prime} \widetilde{y}_{j}^{\prime \prime \prime}$ would have zeros at $\zeta_{\nu-1}$ and $\zeta_{\nu}$, which is impossible. Apply Proposition 6.1 for $x$ and $y$ interchanged. If $0<\bar{\eta}<\widetilde{\eta}_{j}$ is the value which corresponds to $\bar{\xi}$ in Proposition 6.1 then $\bar{\eta} \in \widetilde{y}_{j}(Z)$ by ( $\left.\mathrm{Z}^{\dagger} 4\right)$. Furthermore there is some $1 \leq \nu^{\prime \prime} \leq n$ with $\zeta_{0}<\zeta_{\nu^{\prime \prime}}<\widetilde{x}_{j}(\bar{\eta})$. Consequently, $\zeta_{1}<\widetilde{x}_{j}(\bar{\eta})$ and $\widetilde{y}_{j}\left(\zeta_{1}\right)>\bar{\eta}$. Therefore for sufficiently large $A_{j}$ we have $\bar{\eta} \leq y_{j}\left(\tau_{j}, \zeta\right)=: \eta \leq \eta_{j}\left(\tau_{j}\right)$. Proposition 6.1 gives

$$
\begin{aligned}
R= & \int_{A_{j}^{1 / d_{j}} \eta}^{A_{j}^{1 / d_{j}} \eta_{j}\left(\tau_{j}\right)} A_{j}^{1 / d_{j}} x_{j}\left(\tau_{j}, \tau_{j} y\right) d y+\psi\left(A_{j}^{1 / d_{j}} \eta\right) A_{j}^{1 / d_{j}} x_{j}\left(\tau_{j}, \eta\right) \\
& -\frac{1}{2} A_{j}^{1 / d_{j}}\left(\eta_{j}\left(\tau_{j}\right)-\eta\right)+O\left(A_{j}^{46 /\left(73 d_{j}\right)}\left(\log A_{j}\right)^{315 / 146}\right) \\
& +\left[A_{j}^{1 / d_{j}} \zeta\right]\left[A_{j}^{1 / d_{j}} y_{j}\left(\tau_{j}, \zeta\right)\right] .
\end{aligned}
$$


Substituting $y=A_{j}^{1 / d_{j}} y_{j}\left(\tau_{j}, x\right)$ and partial integration give

$$
\begin{aligned}
R= & A_{j}^{2 / d_{j}} \int_{0}^{\zeta} y_{j}\left(\tau_{j}, x\right) d x-\frac{1}{2} A_{j}^{1 / d_{j}} \zeta-\psi\left(A_{j}^{1 / d_{j}} \zeta\right) A_{j}^{1 / d_{j}} y_{j}\left(\tau_{j}, \zeta\right) \\
& +\psi\left(A_{j}^{1 / d_{j}} 0\right) A_{j}^{1 / d_{j}} y_{j}\left(\tau_{j}, 0\right)+O\left(A_{j}^{46 /\left(73 d_{j}\right)}\left(\log A_{j}\right)^{315 / 146}\right) .
\end{aligned}
$$

LEMmA 7.2. Let $j \in\{1,2\}$ and $1 \leq \nu \leq n$ with $\zeta_{\nu}<\widetilde{\xi}_{j}$. There is some constant $K>0$ so that for $A_{j} \geq K, \zeta \in\left(\zeta_{\nu-1}, \zeta_{\nu}\right]$ with $\left|\zeta-\zeta_{\nu}\right| \ll \tau_{j}$ the asymptotics (7.1) hold. $T\left(A_{j}\right)$ is of the form (7.2) and can only appear if $\widetilde{y}_{j}^{\prime \prime}$ has a zero of order $\mu$ at $\zeta_{\nu-1}$ or $\zeta_{\nu}$ and $\widetilde{y}_{j}^{\prime}$ is rational at this point. $U\left(A_{j}\right)$ is of the form (7.3) and can only appear if $\widetilde{y}_{j}^{\prime \prime}$ has a zero of order $\mu$ at $\zeta_{\nu-1}$ or $\zeta_{\nu}$ and $\widetilde{y}_{j}^{\prime}$ is irrational at this point.

Proof. It is quite similar to that of Lemma 7.1.

CAsE 1: $\widetilde{y}_{j}^{\prime \prime}$ has a zero of order $\mu$ at $\zeta_{\nu-1}$. Apply the reasoning of Case 3 of Lemma 7.1 .

CASE 2: $\widetilde{y}_{j}^{\prime \prime}$ has a zero of order $\mu$ at $\zeta_{\nu}$. From $\left(\mathrm{Z}^{\dagger} 1\right)$ it follows that $\zeta_{\nu} \in Z$. From $\zeta_{0}=0 \in Z$ it follows that $\nu \geq 2$ because there is some $\zeta_{\nu^{\prime}}$ between $\zeta_{0}$ and $\zeta_{\nu}$. Therefore $0<\zeta_{\nu-1}<\zeta_{\nu}<\widetilde{\xi}_{j}$. It follows from (7.6) and $\widetilde{x}_{j}^{\prime}\left(\widetilde{y}_{j}\left(\zeta_{\nu}\right)\right) \neq 0$ that

$$
\left|\widetilde{x}_{j}^{\prime \prime}(y)\right| \asymp\left|\widetilde{y}_{j}^{\prime \prime}\left(\widetilde{x}_{j}(y)\right)\right| \asymp\left|\widetilde{x}_{j}(y)-\zeta_{\nu}\right|^{\mu} \asymp\left|y-\widetilde{y}_{j}\left(\zeta_{\nu}\right)\right|^{\mu}
$$

as $y \rightarrow \widetilde{y}_{j}\left(\zeta_{\nu}\right)$. Therefore $\widetilde{x}_{j}^{\prime \prime}$ has a zero of order $\mu$ at $\widetilde{y}_{j}\left(\zeta_{\nu}\right)$. Furthermore $\widetilde{x}_{j}^{\prime}\left(\widetilde{y}_{j}\left(\zeta_{\nu}\right)\right) \in \mathbb{Q}$ if and only if $\widetilde{y}_{j}^{\prime}\left(\zeta_{\nu}\right) \in \mathbb{Q}$. From the construction of the $\zeta_{\nu}$ it follows that $\widetilde{x}_{j}^{\prime \prime} \widetilde{x}_{j}^{\prime \prime \prime}$ has no zero in $\left(\widetilde{y}_{j}\left(\zeta_{\nu}\right), \widetilde{y}_{j}\left(\zeta_{\nu-1}\right)\right]$. From the assumptions it follows that

$y_{j}\left(\tau_{j}, \zeta\right)=\widetilde{y}_{j}(\zeta)+O\left(\tau_{j}\right)=\widetilde{y}_{j}\left(\zeta_{\nu}\right)+O\left(\tau_{j}\right), \quad y_{j}\left(\tau_{j}, \zeta_{\nu-1}\right)=\widetilde{y}_{j}\left(\zeta_{\nu-1}\right)+O\left(\tau_{j}\right)$.

Applying Proposition 5.2 or Proposition 4.5 to (7.5) gives the desired result.

$\operatorname{CASE} 3: \widetilde{y}_{j}^{\prime \prime}\left(\zeta_{\nu-1}\right) \neq 0 \neq \widetilde{y}_{j}^{\prime \prime}\left(\zeta_{\nu}\right)$.

CAsE 3.1: $\widetilde{y}_{j}^{\prime \prime \prime}\left(\zeta_{\nu-1}\right) \neq 0 \neq \widetilde{y}_{j}^{\prime \prime \prime}\left(\zeta_{\nu}\right)$. Use the reasoning of Case 1 of Lemma 7.1 .

CAsE 3.2: There is some $\nu^{\prime} \in\{\nu-1, \nu\}$ with $\widetilde{y}_{j}^{\prime \prime \prime}\left(\zeta_{\nu^{\prime}}\right)=0$. Use the reasoning of Case 4 of Lemma 7.1 .

The following lemma is used in the construction of the decomposition of $\left[C_{1}, C_{2}\right]$. For $1 \leq \varrho \leq r$ define $\left[a_{\varrho}^{\prime}, b_{\varrho}^{\prime}\right]:=k_{\varrho}^{-1}\left(\left(-\infty, x_{0}\right]\right)$ if the right hand side is nonempty.

LEMma 7.3. For each $\theta \in \mathbb{R}, \kappa>0,1 \leq \varrho \leq r$, there is a decomposition $a_{\varrho}^{\prime}=C_{0}^{*}<\ldots<C_{m}^{*}=b_{\varrho}^{\prime}$ and constants $\varepsilon, K>0$ with the property: If 
$A_{1}, A_{2} \geq K, C:=A_{2} A_{1}^{-d_{2} / d_{1}} \in\left[a_{\varrho}^{\prime}, b_{\varrho}^{\prime}\right],\left|C-C_{\mu}^{*}\right| \geq A_{1}^{-\varepsilon}$ for each $0 \leq \mu \leq m$, then

$$
\left|k_{\varrho}(C)-\theta\right| \geq \tau_{1}^{\kappa}, \quad\left|C^{-1 / d_{2}} k_{\varrho}(C)-\theta\right| \geq \tau_{2}^{\kappa} .
$$

Proof. The function $\widetilde{k}_{\varrho}:\left[a_{\varrho}^{\prime}, b_{\varrho}^{\prime}\right] \rightarrow \mathbb{C}, C \mapsto C^{-1 / d_{2}} k_{\varrho}(C)$, is injective and continuous: If $C \in\left[a_{\varrho}^{\prime}, b_{\varrho}^{\prime}\right], x:=C^{-1 / d_{2}} k_{\varrho}(C)$, then

$$
\widetilde{g}_{2}\left(x, C^{-1 / d_{2}} \widetilde{y}_{1}\left(C^{1 / d_{2}} x\right)\right)=C^{-1} h\left(C^{1 / d_{2}} x\right)=1
$$

and consequently $0 \leq x \leq \widetilde{\xi}_{2}, \widetilde{y}_{1}\left(C^{1 / d_{2}} x\right)=C^{1 / d_{2}} \widetilde{y}_{2}(x)$. The left hand side of the last equation is strictly decreasing in $C$ whereas the right hand side is strictly increasing. Therefore the value $x$ is assumed at no other $\operatorname{argument} C$.

If $\theta$ is not in the range of values of $\widetilde{k}_{\varrho}$ then

$$
\left|C^{-1 / d_{2}} k_{\varrho}(C)-\theta\right| \gg 1 \quad \text { for } C \in\left[a_{\varrho}^{\prime}, b_{\varrho}^{\prime}\right] .
$$

In the opposite case $\theta=\widetilde{k}_{\varrho}\left(C^{*}\right)$ for some $C^{*} \in\left[a_{\varrho}^{\prime}, b_{\varrho}^{\prime}\right]$. Then $k_{\varrho}\left(C^{*}\right) \in$ $\left[0, x_{0}\right] \cap\left[\xi_{\varrho-1}, \xi_{\varrho}\right]$. The function $h$ is holomorphic in some neighbourhood of $\left[0, x_{0}\right]$.

If $h^{\prime}\left(k_{\varrho}\left(C^{*}\right)\right) \neq 0$ then $h$ has a holomorphic inverse in some neighbourhood of $k_{\varrho}\left(C^{*}\right)$ and therefore $k_{\varrho}$ and $\widetilde{k}_{\varrho}$ are holomorphic in some neighbourhood of $C^{*}$. The function $\widetilde{k}_{\varrho}$ is nonconstant. Consequently, there are $p \in \mathbb{N}$ and $\delta>0$ with $\left|\widetilde{k}_{\varrho}(C)-\theta\right| \asymp\left|C-C^{*}\right|^{p}$ for $C \in\left[a_{\varrho}^{\prime}, b_{\varrho}^{\prime}\right],\left|C-C^{*}\right| \leq \delta$. Choosing $0<\varepsilon<\kappa /\left(d_{1} p\right)$ gives $\left|\widetilde{k}_{\varrho}(C)-\theta\right| \gg A_{1}^{-p \varepsilon} \geq \tau_{2}^{\kappa}$ for $C \in\left[a_{\varrho}^{\prime}, b_{\varrho}^{\prime}\right]$ in the case $A_{1}^{-\varepsilon} \leq\left|C-C^{*}\right| \leq \delta$. In the case $\left|C-C^{*}\right| \geq \delta$ we have

$$
\left|\widetilde{k}_{\varrho}(C)-\theta\right| \geq \min _{C^{\prime} \in\left[a_{\varrho}^{\prime}, b_{\varrho}^{\prime}\right],\left|C^{\prime}-C^{*}\right| \geq \delta}\left|\widetilde{k}_{\varrho}\left(C^{\prime}\right)-\theta\right|=\text { const. }>0 .
$$

If $h^{\prime}\left(k_{\varrho}\left(C^{*}\right)\right)=0$ it follows from Lemma 2.1 that there are $p \in \mathbb{N} \backslash\{1\}$ and some neighbourhood $U$ of $k_{\varrho}\left(C^{*}\right)$ with

$$
\left|h(x)-h\left(k_{\varrho}\left(C^{*}\right)\right)\right| \asymp\left|x-k_{\varrho}\left(C^{*}\right)\right|^{p} \quad \text { for } x \in U .
$$

Consequently, there are constants $\delta, K_{i}>0$ with

$$
\begin{aligned}
\left|\widetilde{k}_{\varrho}(C)-\theta\right|= & \left|\left(C^{*}\right)^{-1 / d_{2}} k_{\varrho}(C)-\left(C^{*}\right)^{-1 / d_{2}} k_{\varrho}\left(C^{*}\right)\right| \\
& +O\left(\left|C^{-1 / d_{2}}-\left(C^{*}\right)^{-1 / d_{2}}\right| \cdot\left|k_{\varrho}(C)\right|\right) \\
\geq & K_{3}\left|k_{\varrho}(C)-k_{\varrho}\left(C^{*}\right)\right|-K_{4}\left|C-C^{*}\right| \\
\geq & K_{5}\left|h\left(k_{\varrho}(C)\right)-h\left(k_{\varrho}\left(C^{*}\right)\right)\right|^{1 / p}-K_{4}\left|C-C^{*}\right| \\
= & K_{5}\left|C-C^{*}\right|^{1 / p}-K_{4}\left|C-C^{*}\right| \geq K_{6}\left|C-C^{*}\right|^{1 / p}
\end{aligned}
$$

for $C \in\left[a_{\varrho}^{\prime}, b_{\varrho}^{\prime}\right],\left|C-C^{*}\right| \leq \delta$. The remainder of the argument is as above.

The function $k_{\varrho}$ is injective on $\left[a_{\varrho}^{\prime}, b_{\varrho}^{\prime}\right]$. If $\theta \notin k_{\varrho}\left(\left[a_{\varrho}^{\prime}, b_{\varrho}^{\prime}\right]\right)$ then $\left|k_{\varrho}(C)-\theta\right|$ $\gg 1$ for $C \in\left[a_{\varrho}^{\prime}, b_{\varrho}^{\prime}\right]$. In the opposite case there is some $C^{*} \in\left[a_{\varrho}^{\prime}, b_{\varrho}^{\prime}\right]$ with 
$\theta=k_{\varrho}\left(C^{*}\right) \in\left[0, x_{0}\right]$. From $h \in C^{\infty}\left[0, x_{0}\right]$ it follows that

$$
\left|C-C^{*}\right|=\left|h\left(k_{\varrho}(C)\right)-h\left(k_{\varrho}\left(C^{*}\right)\right)\right| \ll\left|k_{\varrho}(C)-k_{\varrho}\left(C^{*}\right)\right|
$$

for $C \in\left[a_{\varrho}^{\prime}, b_{\varrho}^{\prime}\right]$. The remainder of the argument is as above.

Now use Lemma 7.3 and the injectivity of $k_{\varrho}$ and $\widetilde{k}_{\varrho}$ to construct a decomposition $C_{1}=C_{0}^{*}<\ldots<C_{m}^{*}=C_{2}$ and some $\varepsilon>0$ with the properties:

$\left(\mathrm{C}^{\dagger} 1\right) \quad$ If $a_{\varrho}^{\prime} \in\left[C_{1}, C_{2}\right]$ for some $1 \leq \varrho \leq r$ then $a_{\varrho}^{\prime} \in\left\{C_{0}^{*}, \ldots, C_{m}^{*}\right\}$. The same holds for $a_{\varrho}, b_{\varrho}^{\prime}, b_{\varrho}$.

$\left(\mathrm{C}^{\dagger} 2\right) \quad$ For $1 \leq \varrho \leq r, 1 \leq \mu \leq m, 0 \leq \nu \leq n$, with $\left[C_{\mu-1}^{*}, C_{\mu}^{*}\right] \subseteq\left[a_{\varrho}^{\prime}, b_{\varrho}^{\prime}\right]$ : For $A_{1}, A_{2} \geq K, C:=A_{2} A_{1}^{-d_{2} / d_{1}} \in\left(C_{\mu-1}^{*}+A_{1}^{-\varepsilon}, C_{\mu}^{*}-A_{1}^{-\varepsilon}\right)$ we have

$$
\left|k_{\varrho}(C)-\zeta_{\nu}\right| \geq \tau_{1}^{\kappa}, \quad\left|C^{-1 / d_{2}} k_{\varrho}(C)-\zeta_{\nu}\right| \geq \tau_{2}^{\kappa} .
$$

$\left(\mathrm{C}^{\dagger} 3\right) \quad$ For $1 \leq \mu \leq m, 1 \leq \nu^{\prime} \leq n, 0 \leq \nu \leq n$ : For $A_{1}, A_{2} \geq K, C:=$ $A_{2} A_{1}^{-d_{2} / d_{1}} \in\left(C_{\mu-1}^{*}+A_{1}^{-\varepsilon}, C_{\mu}^{*}-A_{1}^{-\varepsilon}\right)$ we have $\left|C^{-1 / d_{2}} \zeta_{\nu^{\prime}}-\zeta_{\nu}\right| \geq \tau_{2}^{\kappa}$.

$\left(\mathrm{C}^{\dagger} 4\right) \quad$ For $1 \leq \varrho \leq r, 1 \leq \mu \leq m, 1 \leq \nu^{\prime} \leq n, 0 \leq \nu \leq n$ we have

$$
\begin{gathered}
\zeta_{\nu} \notin k_{\varrho}\left(\left(C_{\mu-1}^{*}, C_{\mu}^{*}\right) \cap\left[a_{\varrho}^{\prime}, b_{\varrho}^{\prime}\right]\right), \quad \zeta_{\nu} \notin \widetilde{k}_{\varrho}\left(\left(C_{\mu-1}^{*}, C_{\mu}^{*}\right) \cap\left[a_{\varrho}^{\prime}, b_{\varrho}^{\prime}\right]\right), \\
\zeta_{\nu} \neq C^{-1 / d_{2}} \zeta_{\nu^{\prime}} \quad \text { for } C \in\left(C_{\mu-1}^{*}, C_{\mu}^{*}\right) .
\end{gathered}
$$

With this decomposition the following lemma holds which contains one half of Theorem 1.1.

Lemma 7.4. Let $1 \leq \mu \leq m, 1 \leq \varrho \leq r$ with $\xi_{\varrho-1}<x_{0}$. Then for $A_{1}, A_{2} \geq K, C:=A_{2} A_{1}^{-d_{2} / d_{1}} \in\left(C_{\mu-1}^{*}+A_{1}^{-\varepsilon}, C_{\mu}^{*}-A_{1}^{-\varepsilon}\right)$ we have

$$
\begin{aligned}
R_{\varrho}^{\dagger}\left(A_{1}, A_{2}\right)= & \int_{A_{1}^{1 / d_{1}} \xi_{\varrho-1}}^{A_{1}^{1 / d_{1}} \min \left\{\xi_{\varrho}, x_{0}\right\}} f_{A_{1}, A_{2}}(x) d x \\
& -\frac{1}{2} A_{1}^{1 / d_{1}}\left(\min \left\{\xi_{\varrho}, x_{0}\right\}-\xi_{\varrho-1}\right)+T_{1}^{\dagger(\mu, \varrho)}\left(A_{1}\right) \\
& +T_{2}^{\dagger(\mu, \varrho)}\left(A_{2}\right)+U_{1}^{\dagger(\mu, \varrho)}\left(A_{1}\right)+U_{2}^{\dagger(\mu, \varrho)}\left(A_{2}\right) \\
& -\psi\left(A_{1}^{1 / d_{1}} \min \left\{\xi_{\varrho}, x_{0}\right\}\right) f_{A_{1}, A_{2}}\left(A_{1}^{1 / d_{1}} \min \left\{\xi_{\varrho}, x_{0}\right\}\right) \\
& +\psi\left(A_{1}^{1 / d_{1}} \xi_{\varrho}\right) f_{A_{1}, A_{2}}\left(A_{1}^{1 / d_{1}} \xi_{\varrho-1}\right) \\
& +O\left(A_{1}^{46 /\left(73 d_{1}\right)}\left(\log A_{1}\right)^{315 / 146}\right) .
\end{aligned}
$$

The functions $T_{j}^{\dagger(\mu, \varrho)}$ and $U_{j}^{\dagger(\mu, \varrho)}$ are of the form described in Theorem 1.1.

Pr o of. Several cases have to be distinguished in order to apply Lemma 3.1. 
CAse 1: $\left[C_{\mu-1}^{*}, C_{\mu}^{*}\right] \subseteq\left[a_{\varrho}^{\prime}, b_{\varrho}^{\prime}\right]$. From $\left(\mathrm{Z}^{\dagger} 3\right),\left(\mathrm{Z}^{\dagger} 5\right)$ and $\left(\mathrm{C}^{\dagger} 4\right)$ it follows that $x_{0}$ is not contained in the interval $k_{\varrho}\left(\left(C_{\mu-1}^{*}, C_{\mu}^{*}\right)\right)$.

CASE 1.1: $k_{\varrho}\left(\left(C_{\mu-1}^{*}, C_{\mu}^{*}\right)\right) \subseteq\left(-\infty, x_{0}\right)$. Then $\xi_{\varrho-1} \leq k_{\varrho}(C)<x_{0}$.

Lemma 3.1 gives the decomposition in the following two cases.

CASE 1.1.1: If $h \uparrow\left[\xi_{\varrho-1}, \xi_{\varrho}\right]$ is increasing then

$$
\begin{aligned}
R_{\varrho}^{\dagger}= & \#\left\{(x, y) \in \mathbb{Z}^{2} \mid A_{1}^{1 / d_{1}} \xi_{\varrho-1}<x \leq A_{1}^{1 / d_{1}} k_{\varrho}(C),\right. \\
& \left.0<y \leq A_{1}^{1 / d_{1}} y_{1}\left(\tau_{1}, \tau_{1} x\right)\right\} \\
& +\#\left\{(x, y) \in \mathbb{Z}^{2} \mid A_{1}^{1 / d_{1}} k_{\varrho}(C)<x \leq A_{1}^{1 / d_{1}} \min \left\{\xi_{\varrho}, x_{0}\right\},\right. \\
& \left.0<y \leq A_{2}^{1 / d_{2}} y_{2}\left(\tau_{2}, \tau_{2} x\right)\right\} \\
& +O\left(A_{1}^{46 /\left(73 d_{1}\right)}\right) .
\end{aligned}
$$

CASE 1.1.2: If $h \uparrow\left[\xi_{\varrho-1}, \xi_{\varrho}\right]$ is decreasing then

$$
\begin{aligned}
R_{\varrho}^{\dagger}= & \#\left\{(x, y) \in \mathbb{Z}^{2} \mid A_{1}^{1 / d_{1}} \xi_{\varrho-1}<x \leq A_{1}^{1 / d_{1}} k_{\varrho}(C),\right. \\
& \left.0<y \leq A_{2}^{1 / d_{2}} y_{2}\left(\tau_{2}, \tau_{2} x\right)\right\} \\
& +\#\left\{(x, y) \in \mathbb{Z}^{2} \mid A_{1}^{1 / d_{1}} k_{\varrho}(C)<x \leq A_{1}^{1 / d_{1}} \min \left\{\xi_{\varrho}, x_{0}\right\},\right. \\
& \left.0<y \leq A_{1}^{1 / d_{1}} y_{1}\left(\tau_{1}, \tau_{1} x\right)\right\} \\
& +O\left(A_{1}^{46 /\left(73 d_{1}\right)}\right) .
\end{aligned}
$$

CASE 1.2: $k_{\varrho}\left(\left(C_{\mu-1}^{*}, C_{\mu}^{*}\right)\right) \subseteq\left(x_{0}, \infty\right)$. Then $\xi_{\varrho-1}<x_{0}<k_{\varrho}(C) \leq \xi_{\varrho}$. Lemma 3.1 gives the decomposition in the following two cases.

CASE 1.2.1: If $h \uparrow\left[\xi_{\varrho-1}, \xi_{\varrho}\right]$ is increasing then

$$
\begin{aligned}
R_{\varrho}^{\dagger}= & \#\left\{(x, y) \in \mathbb{Z}^{2} \mid A_{1}^{1 / d_{1}} \xi_{\varrho-1}<x \leq A_{1}^{1 / d_{1}} x_{0}, 0<y \leq A_{1}^{1 / d_{1}} y_{1}\left(\tau_{1}, \tau_{1} x\right)\right\} \\
& +O\left(A_{1}^{46 /\left(73 d_{1}\right)}\right) .
\end{aligned}
$$

CASE 1.2.2: If $h \uparrow\left[\xi_{\varrho-1}, \xi_{\varrho}\right]$ is decreasing then

$$
\begin{aligned}
R_{\varrho}^{\dagger}= & \#\left\{(x, y) \in \mathbb{Z}^{2} \mid A_{1}^{1 / d_{1}} \xi_{\varrho-1}<x \leq A_{1}^{1 / d_{1}} x_{0}, 0<y \leq A_{2}^{1 / d_{2}} y_{2}\left(\tau_{2}, \tau_{2} x\right)\right\} \\
& +O\left(A_{1}^{46 /\left(73 d_{1}\right)}\right) .
\end{aligned}
$$

CASE 2: $\left[C_{\mu-1}^{*}, C_{\mu}^{*}\right] \subseteq\left[a_{\varrho}, a_{\varrho}^{\prime}\right]$ or $\left[C_{\mu-1}^{*}, C_{\mu}^{*}\right] \subseteq\left[b_{\varrho}^{\prime}, b_{\varrho}\right]$. Then $C \in\left[a_{\varrho}, b_{\varrho}\right]$ and $k_{\varrho}(C)>x_{0}$. For $R_{\varrho}^{\dagger}$ the same holds as in Case 1.2.

CASE 3: $C_{\mu}^{*} \leq a_{\varrho}$. Then by Lemma 3.1

$$
\begin{aligned}
R_{\varrho}^{\dagger}=\#\left\{(x, y) \in \mathbb{Z}^{2} \mid A_{1}^{1 / d_{1}} \xi_{\varrho-1}<x \leq A_{1}^{1 / d_{1}} \min \left\{\xi_{\varrho}, x_{0}\right\},\right. \\
\left.0<y \leq A_{2}^{1 / d_{2}} y_{2}\left(\tau_{2}, \tau_{2} x\right)\right\} .
\end{aligned}
$$


CASE 4: $C_{\mu-1}^{*} \geq b_{\varrho}$. Then by Lemma 3.1

$$
\begin{aligned}
R_{\varrho}^{\dagger}=\#\left\{(x, y) \in \mathbb{Z}^{2} \mid A_{1}^{1 / d_{1}} \xi_{\varrho-1}<x \leq A_{1}^{1 / d_{1}} \min \left\{\xi_{\varrho}, x_{0}\right\},\right. \\
\left.0<y \leq A_{1}^{1 / d_{1}} y_{1}\left(\tau_{1}, \tau_{1} x\right)\right\} .
\end{aligned}
$$

In the following the proof of (7.7) is given only in Cases 1.1.1 and 1.1.2. The other cases are similar but somewhat easier.

In Case 1.1.1 use $\left(\mathrm{C}^{\dagger} 4\right),(2.7)$ and $\left(\mathrm{Z}^{\dagger} 3\right)$ and choose $1 \leq \nu_{j} \leq n$ with the properties

$$
\begin{gathered}
k_{\varrho}\left(\left(C_{\mu-1}^{*}, C_{\mu}^{*}\right)\right) \subseteq\left(\zeta_{\nu_{0}-1}, \zeta_{\nu_{0}}\right) \subseteq\left(\xi_{\varrho-1}, \min \left\{\xi_{\varrho}, x_{0}\right\}\right), \quad \xi_{\varrho-1}=\zeta_{\nu_{1}-1}, \\
\min \left\{\xi_{\varrho}, x_{0}\right\}=\zeta_{\nu_{2}}, \quad \nu_{1} \leq \nu_{0} \leq \nu_{2}, \quad \widetilde{k}_{\varrho}\left(\left(C_{\mu-1}^{*}, C_{\mu}^{*}\right)\right) \subseteq\left(\zeta_{\nu_{3}-1}, \zeta_{\nu_{3}}\right), \\
C^{-1 / d_{2}} \zeta_{\nu_{2}} \in\left(\zeta_{\nu_{4}-1}, \zeta_{\nu_{4}}\right) \subseteq\left[0, x_{2}\right] \quad \text { for } C \in\left(C_{\mu-1}^{*}, C_{\mu}^{*}\right), \quad \nu_{3} \leq \nu_{4} .
\end{gathered}
$$

Then

$$
\begin{aligned}
R_{\varrho}^{\dagger}=\sum_{\nu=\nu_{1}}^{\nu_{0}-1} \#\left\{(x, y) \in \mathbb{Z}^{2} \mid A_{1}^{1 / d_{1}} \zeta_{\nu-1}<x \leq A_{1}^{1 / d_{1}} \zeta_{\nu},\right. \\
\left.\quad 0<y \leq A_{1}^{1 / d_{1}} y_{1}\left(\tau_{1}, \tau_{1} x\right)\right\} \\
+\#\left\{(x, y) \in \mathbb{Z}^{2} \mid A_{1}^{1 / d_{1}} \zeta_{\nu_{0}-1}<x \leq A_{1}^{1 / d_{1}} k_{\varrho}(C),\right. \\
\left.0<y \leq A_{1}^{1 / d_{1}} y_{1}\left(\tau_{1}, \tau_{1} x\right)\right\} \\
+\#\left\{(x, y) \in \mathbb{Z}^{2} \mid A_{2}^{1 / d_{2}} \zeta_{\nu_{3}-1}<x \leq A_{2}^{1 / d_{2}} C^{-1 / d_{2}} k_{\varrho}(C),\right. \\
\left.0<y \leq A_{2}^{1 / d_{2}} y_{2}\left(\tau_{2}, \tau_{2} x\right)\right\} \\
+\sum_{\nu=\nu_{3}}^{\nu_{4}-1} \#\left\{(x, y) \in \mathbb{Z}^{2} \mid A_{2}^{1 / d_{2}} \zeta_{\nu-1}<x \leq A_{2}^{1 / d_{2}} \zeta_{\nu},\right. \\
+\#\left\{(x, y) \in \mathbb{Z}^{2} \mid A_{2}^{1 / d_{2}} \zeta_{\nu_{4}-1}<x \leq A_{2}^{1 / d_{2}} C^{-1 / d_{2}} \zeta_{\nu_{2}},\right. \\
\left.0<y \leq A_{2}^{1 / d_{2}} y_{2}\left(\tau_{2}, \tau_{2} x\right)\right\} \\
+O\left(A_{1}^{46 /\left(73 d_{1}\right)}\right) .
\end{aligned}
$$

It follows from $\left(\mathrm{C}^{\dagger} 2\right)$ and $\left(\mathrm{C}^{\dagger} 3\right)$ that the endpoints of the respective intervals of $x$ when renormalized with the corresponding $A_{j}^{-1 / d_{j}}$ have a distance $\geq \tau_{j}^{\kappa}$ from all $\zeta_{\nu}$ with $0 \leq \nu \leq n$, or are equal to one of these $\zeta_{\nu}$. Furthermore $\zeta_{\nu_{0}}<\widetilde{\xi}_{1}, \zeta_{\nu_{4}}<\widetilde{\xi}_{2}$. Application of Lemmas 7.1 and 7.2 to each of the summands gives

$$
\begin{aligned}
R_{\varrho}^{\dagger}= & A_{1}^{2 / d_{1}} \int_{\zeta_{\nu_{1}-1}}^{k_{\varrho}(C)} y_{1}\left(\tau_{1}, x\right) d x+A_{2}^{2 / d_{2}} \int_{C^{-1 / d_{2}} k_{\varrho}(C)}^{C^{-1 / d_{2}} \zeta_{\nu_{2}}} y_{2}\left(\tau_{2}, x\right) d x \\
& +\frac{1}{2} A_{1}^{1 / d_{1}} \zeta_{\nu_{1}-1}-\frac{1}{2} A_{2}^{1 / d_{2}} C^{-1 / d_{2}} \zeta_{\nu_{2}} \\
& +T_{1}^{\dagger(\mu, \varrho)}\left(A_{1}\right)+T_{2}^{\dagger(\mu, \varrho)}\left(A_{2}\right)
\end{aligned}
$$




$$
\begin{aligned}
& +\psi\left(A_{1}^{1 / d_{1}} \zeta_{\nu_{1}-1}\right) A_{1}^{1 / d_{1}} y_{1}\left(\tau_{1}, \zeta_{\nu_{1}-1}\right) \\
& -\psi\left(A_{1}^{1 / d_{1}} k_{\varrho}(C)\right) A_{1}^{1 / d_{1}} y_{1}\left(\tau_{1}, k_{\varrho}(C)\right) \\
& +\psi\left(A_{2}^{1 / d_{2}} C^{-1 / d_{2}} k_{\varrho}(C)\right) A_{2}^{1 / d_{2}} y_{2}\left(\tau_{2}, C^{-1 / d_{2}} k_{\varrho}(C)\right) \\
& +U_{1}^{\dagger(\mu, \varrho)}\left(A_{1}\right)+U_{2}^{\dagger(\mu, \varrho)}\left(A_{2}\right) \\
& -\psi\left(A_{2}^{1 / d_{2}} C^{-1 / d_{2}} \zeta_{\nu_{2}}\right) A_{2}^{1 / d_{2}} y_{2}\left(\tau_{2}, C^{-1 / d_{2}} \zeta_{\nu_{2}}\right) \\
& +O\left(A_{1}^{46 /\left(73 d_{1}\right)}\left(\log A_{1}\right)^{315 / 146}\right) .
\end{aligned}
$$

Lemma 2.2, (3.3) and (3.5) give for the first integral

$$
\begin{gathered}
\int_{\xi_{\varrho-1}}^{k_{\varrho}(C)-\tau_{1}^{50 / 73}} \tau_{1} f_{A_{1}, A_{2}}\left(A_{1}^{1 / d_{1}} x\right) d x \\
+\int_{k_{\varrho}(C)-\tau_{1}^{50 / 73}}^{k_{\varrho}(C)}\left(\tau_{1} f_{A_{1}, A_{2}}\left(A_{1}^{1 / d_{1}} x\right)+O\left(\tau_{1}^{50 / 73}\right)\right) d x,
\end{gathered}
$$

for the second integral

$$
\begin{gathered}
C^{-1 / d_{2}} \int_{k_{\varrho}(C)}^{k_{\varrho}(C)+\tau_{1}^{50 / 73}}\left(\tau_{2} f_{A_{1}, A_{2}}\left(A_{1}^{1 / d_{1}} x\right)+O\left(\tau_{2}^{50 / 73}\right)\right) d x \\
+C^{-1 / d_{2}} \int_{k_{\varrho}(C)+\tau_{1}^{50 / 73}}^{\zeta_{\nu_{2}}} \tau_{2} f_{A_{1}, A_{2}}\left(A_{1}^{1 / d_{1}} x\right) d x
\end{gathered}
$$

and

$$
\begin{array}{r}
y_{1}\left(\tau_{1}, k_{\varrho}(C)\right)=\tau_{1} f_{A_{1}, A_{2}}\left(A_{1}^{1 / d_{1}} k_{\varrho}(C)\right)+O\left(\tau_{1}^{50 / 73}\right), \\
y_{2}\left(\tau_{2}, C^{-1 / d_{2}} k_{\varrho}(C)\right)=\tau_{2} f_{A_{1}, A_{2}}\left(A_{1}^{1 / d_{1}} k_{\varrho}(C)\right)+O\left(\tau_{2}^{50 / 73}\right) .
\end{array}
$$

Substituting into (7.8) gives (7.7).

In Case 1.1.2 choose $1 \leq \nu_{j} \leq n$ with the properties

$$
\begin{gathered}
k_{\varrho}\left(\left(C_{\mu-1}^{*}, C_{\mu}^{*}\right)\right) \subseteq\left(\zeta_{\nu_{0}-1}, \zeta_{\nu_{0}}\right) \subseteq\left(\xi_{\varrho-1}, \min \left\{\xi_{\varrho}, x_{0}\right\}\right), \\
\xi_{\varrho-1}=\zeta_{\nu_{1}-1}, \quad \min \left\{\xi_{\varrho}, x_{0}\right\}=\zeta_{\nu_{2}}, \quad \nu_{1} \leq \nu_{0} \leq \nu_{2}, \quad \zeta_{\nu_{2}}<\widetilde{\xi}_{1}, \\
\widetilde{k}_{\varrho}\left(\left(C_{\mu-1}^{*}, C_{\mu}^{*}\right)\right) \subseteq\left(\zeta_{\nu_{3}-1}, \zeta_{\nu_{3}}\right) \subseteq\left[0, x_{2}\right], \quad \zeta_{\nu_{3}}<\widetilde{\xi}_{2}, \\
C^{-1 / d_{2}} \zeta_{\nu_{1}-1} \in\left(\zeta_{\nu_{4}-1}, \zeta_{\nu_{4}}\right) \text { or } \equiv \zeta_{\nu_{4}-1}(\text { if } \varrho=1) \\
\text { for } C \in\left(C_{\mu-1}^{*}, C_{\mu}^{*}\right), \nu_{4} \leq \nu_{3} .
\end{gathered}
$$

Then

$$
\begin{array}{r}
R_{\varrho}^{\dagger}=-\#\left\{(x, y) \in \mathbb{Z}^{2} \mid A_{2}^{1 / d_{2}} \zeta_{\nu_{4}-1}<x \leq A_{2}^{1 / d_{2}} C^{-1 / d_{2}} \zeta_{\nu_{1}-1},\right. \\
\left.0<y \leq A_{2}^{1 / d_{2}} y_{2}\left(\tau_{2}, \tau_{2} x\right)\right\}
\end{array}
$$




$$
\begin{aligned}
& +\sum_{\nu=\nu_{4}}^{\nu_{3}-1} \#\left\{(x, y) \in \mathbb{Z}^{2} \mid A_{2}^{1 / d_{2}} \zeta_{\nu-1}<x \leq A_{2}^{1 / d_{2}} \zeta_{\nu},\right. \\
& \left.0<y \leq A_{2}^{1 / d_{2}} y_{2}\left(\tau_{2}, \tau_{2} x\right)\right\} \\
& +\#\left\{(x, y) \in \mathbb{Z}^{2} \mid A_{2}^{1 / d_{2}} \zeta_{\nu_{3}-1}<x \leq A_{2}^{1 / d_{2}} C^{-1 / d_{2}} k_{\varrho}(C),\right. \\
& \left.0<y \leq A_{2}^{1 / d_{2}} y_{2}\left(\tau_{2}, \tau_{2} x\right)\right\} \\
& +\#\left\{(x, y) \in \mathbb{Z}^{2} \mid A_{1}^{1 / d_{1}} \zeta_{\nu_{0}-1}<x \leq A_{1}^{1 / d_{1}} k_{\varrho}(C),\right. \\
& \left.0<y \leq A_{1}^{1 / d_{1}} y_{1}\left(\tau_{1}, \tau_{1} x\right)\right\} \\
& +\sum_{\nu=\nu_{0}} \#\left\{(x, y) \in \mathbb{Z}^{2} \mid A_{1}^{1 / d_{1}} \zeta_{\nu-1}<x \leq A_{1}^{1 / d_{1}} \zeta_{\nu},\right. \\
& +O\left(A_{1}^{46 /\left(73 d_{1}\right)}\right) .
\end{aligned}
$$

The remainder of the proof is as above.

Summing over $1 \leq \varrho \leq r$ gives

Corollary 7.5. Let $1 \leq \mu \leq m$. Then for $A_{1}, A_{2} \geq K, C:=A_{2} A_{1}^{-d_{2} / d_{1}}$ $\in\left(C_{\mu-1}^{*}+A_{1}^{-\varepsilon}, C_{\mu}^{*}-A_{1}^{-\varepsilon}\right)$ we have

$$
\begin{aligned}
R^{\dagger}\left(A_{1}, A_{2}\right)= & \int_{0}^{A_{1}^{1 / d_{1}} x_{0}} f_{A_{1}, A_{2}}(x) d x-\frac{1}{2} A_{1}^{1 / d_{1}} x_{0}+T_{1}^{\dagger(\mu)}\left(A_{1}\right)+T_{2}^{\dagger(\mu)}\left(A_{2}\right) \\
& +U_{1}^{\dagger(\mu)}\left(A_{1}\right)+U_{2}^{\dagger(\mu)}\left(A_{2}\right)-\frac{1}{2} f_{A_{1}, A_{2}}(0) \\
& -\psi\left(A_{1}^{1 / d_{1}} x_{0}\right) f_{A_{1}, A_{2}}\left(A_{1}^{1 / d_{1}} x_{0}\right) \\
& +O\left(A_{1}^{46 /\left(73 d_{1}\right)}\left(\log A_{1}\right)^{315 / 146}\right) .
\end{aligned}
$$

Now $R^{\#}$ is handled similarly to $R^{\dagger}$ but with the roles of $x$ and $y$ interchanged. The analogue of $h:\left[0, \widetilde{\xi}_{1}\right] \rightarrow \mathbb{R}^{+}$is $l:\left[0, \widetilde{\eta}_{1}\right] \rightarrow \mathbb{R}^{+}$with $l(y):=\widetilde{g}_{2}\left(\widetilde{x}_{1}(y), y\right)$. Let $0=\eta_{0}<\ldots<\eta_{t}=\widetilde{\eta}_{1}$ be the decomposition which is analogous to $0=\xi_{0}<\ldots<\xi_{r}=\widetilde{\xi}_{1}$ and $m_{\tau}:=\left(l \uparrow\left[\eta_{\tau-1}, \eta_{\tau}\right]\right)^{-1}$ : $\left[c_{\tau}, d_{\tau}\right] \rightarrow\left[\eta_{\tau-1}, \eta_{\tau}\right]$ for $1 \leq \tau \leq t$.

The situations for $R^{\dagger}$ and $R^{\#}$ are not completely symmetric because $y_{0}=y_{0, A_{1}, A_{2}}$ depends on $A_{1}$ and $A_{2}$ whereas $x_{0}$ is constant. Therefore some additional lemmas are needed.

Lemma 7.6. For $\bar{y}, \kappa>0$ there is a decomposition $C_{1}=C_{0}^{*}<\ldots<$ $C_{m}^{*}=C_{2}$ and constants $\varepsilon, K>0$ so that for $1 \leq \mu \leq m$ the following holds:

(1) Uniformly for all $A_{1}, A_{2} \geq K$ with $C:=A_{2} A_{1}^{-d_{2} / d_{1}} \in\left(C_{\mu-1}^{*}+A_{1}^{-\varepsilon}\right.$, $\left.C_{\mu}^{*}-A_{1}^{-\varepsilon}\right)$, one of the two cases $y_{0, A_{1}, A_{2}} \leq \bar{y}$ or $y_{0, A_{1}, A_{2}}>\bar{y}$ is valid. 
(2) Uniformly for all $A_{1}, A_{2} \geq K$ with $C:=A_{2} A_{1}^{-d_{2} / d_{1}} \in\left(C_{\mu-1}^{*}+A_{1}^{-\varepsilon}\right.$, $\left.C_{\mu}^{*}-A_{1}^{-\varepsilon}\right)$ one of the two estimates $\left|y_{0, A_{1}, A_{2}}-\bar{y}\right| \geq \tau_{1}^{\kappa}$ or $\left|y_{0, A_{1}, A_{2}}-\bar{y}\right| \ll \tau_{1}$ is valid.

Proof. Choose $\widetilde{g}_{2}\left(x_{0}, \bar{y}\right)$ as point of decomposition if it lies in $\left[C_{1}, C_{2}\right]$.

CASE 1: $C_{\mu}^{*} \leq \widetilde{g}_{2}\left(x_{0}, \bar{y}\right)$. Then $C \leq \widetilde{g}_{2}\left(x_{0}, \bar{y}\right)-A_{1}^{-\varepsilon}$ and consequently

$$
\begin{aligned}
\widetilde{g}_{2}\left(C^{-1 / d_{2}} x_{0}, C^{-1 / d_{2}} \bar{y}\right) & \geq 1+C^{-1} A_{1}^{-\varepsilon} \\
& \geq \widetilde{g}_{2}\left(C^{-1 / d_{2}} x_{0}, \widetilde{y}_{2}\left(C^{-1 / d_{2}} x_{0}\right)\right)+C_{2}^{-1} A_{1}^{-\varepsilon} .
\end{aligned}
$$

Therefore $C^{-1 / d_{2}} \bar{y}>\widetilde{y}_{2}\left(C^{-1 / d_{2}} x_{0}\right)$ and

$$
\begin{aligned}
& \left|C^{-1 / d_{2}} \bar{y}-\widetilde{y}_{2}\left(C^{-1 / d_{2}} x_{0}\right)\right| \\
& \quad \gg\left|\widetilde{g}_{2}\left(C^{-1 / d_{2}} x_{0}, C^{-1 / d_{2}} \bar{y}\right)-\widetilde{g}_{2}\left(C^{-1 / d_{2}} x_{0}, \widetilde{y}_{2}\left(C^{-1 / d_{2}} x_{0}\right)\right)\right| \gg A_{1}^{-\varepsilon} .
\end{aligned}
$$

It follows that $\bar{y} \geq C^{1 / d_{2}} \widetilde{y}_{2}\left(C^{-1 / d_{2}} x_{0}\right)+K_{30} A_{1}^{-\varepsilon}$ with some constant $K_{30}>0$. From the definition of $y_{0, A_{1}, A_{2}}$ it follows that

$$
y_{0, A_{1}, A_{2}} \leq C^{1 / d_{2}} y_{2}\left(\tau_{2}, C^{-1 / d_{2}} x_{0}\right) \leq C^{1 / d_{2}} \widetilde{y}_{2}\left(C^{-1 / d_{2}} x_{0}\right) \leq \bar{y}-K_{30} A_{1}^{-\varepsilon} .
$$

Choosing $0<\varepsilon<\kappa / d_{1}$ it follows that in (1) and (2) always the first cases are valid.

CASE 2: $C_{\mu-1}^{*} \geq \tilde{g}_{2}\left(x_{0}, \bar{y}\right)$. The same reasoning as above gives $C^{1 / d_{2}} \widetilde{y}_{2}\left(C^{-1 / d_{2}} x_{0}\right) \geq \bar{y}+K_{30} A_{1}^{-\varepsilon}$ with some constant $K_{30}>0$.

CASE 2.1: $\widetilde{y}_{1}\left(x_{0}\right)>\bar{y}$. Then with some constant $K_{31}>0$,

$$
\begin{aligned}
y_{0, A_{1}, A_{2}} & =\min \left\{y_{1}\left(\tau_{1}, x_{0}\right), C^{1 / d_{2}} y_{2}\left(\tau_{2}, C^{-1 / d_{2}} x_{0}\right)\right\} \\
& \geq \min \left\{\widetilde{y}_{1}\left(x_{0}\right)-K_{31} \tau_{1}, C^{1 / d_{2}} \widetilde{y}_{2}\left(C^{-1 / d_{2}} x_{0}\right)-K_{31} \tau_{1}\right\} \\
& \geq \bar{y}+K_{30} A_{1}^{-\varepsilon} / 2
\end{aligned}
$$

and so if $0<\varepsilon<\min \left\{1 / d_{1}, \kappa / d_{1}\right\}$ is chosen then in (1) always the second case is valid and in (2) always the first case.

CASE 2.2: $\widetilde{y}_{1}\left(x_{0}\right)=\bar{y}$. Then with some constant $K_{32}>0$ we have

$$
\begin{aligned}
C^{1 / d_{2}} y_{2}\left(\tau_{2}, C^{-1 / d_{2}} x_{0}\right) & \geq C^{1 / d_{2}} \widetilde{y}_{2}\left(C^{-1 / d_{2}} x_{0}\right)-K_{32} \tau_{1} \\
& \geq \widetilde{y}_{1}\left(x_{0}\right)-K_{32} \tau_{1}+K_{30} A_{1}^{-\varepsilon} \geq y_{1}\left(\tau_{1}, x_{0}\right)
\end{aligned}
$$

and so $y_{0}=y_{1}\left(\tau_{1}, x_{0}\right)$. Consequently, $y_{0} \leq \widetilde{y}_{1}\left(x_{0}\right)=\bar{y}$ and $\left|y_{0, A_{1}, A_{2}}-\bar{y}\right|=$ $\left|y_{1}\left(\tau_{1}, x_{0}\right)-\widetilde{y}_{1}\left(x_{0}\right)\right| \ll \tau_{1}$. So in (1) always the first case and in (2) always the second case is valid.

CASE 2.3: $\widetilde{y}_{1}\left(x_{0}\right)<\bar{y}$. Then $y_{0, A_{1}, A_{2}} \leq y_{1}\left(\tau_{1}, x_{0}\right) \leq \widetilde{y}_{1}\left(x_{0}\right)<\bar{y}$ and $\left|y_{0, A_{1}, A_{2}}-\bar{y}\right| \geq \bar{y}-\widetilde{y}_{1}\left(x_{0}\right)=$ const. $>0$. So in (1) and (2) always the first cases are valid. 
Lemma 7.7. For $\bar{y}, \kappa>0$ there is a decomposition $C_{1}=C_{0}^{*}<\ldots<C_{m}^{*}$ $=C_{2}$ and $K, \varepsilon>0$ so that for $1 \leq \mu \leq m$ and uniformly for $A_{1}, A_{2} \geq K$ with $C:=A_{2} A_{1}^{-d_{2} / d_{1}} \in\left(C_{\mu-1}^{*}+A_{1}^{-\varepsilon}, C_{\mu}^{*}-A_{1}^{-\varepsilon}\right)$ one of the two cases $C^{-1 / d_{2}} y_{0, A_{1}, A_{2}} \geq \bar{y}+\tau_{1}^{\kappa}$ or $C^{-1 / d_{2}} y_{0, A_{1}, A_{2}} \leq \bar{y}-\tau_{1}^{\kappa}$ is valid.

Proof. CASE $1: \bar{y} \geq \widetilde{\eta}_{2}$. Then

$$
\begin{aligned}
\bar{y}-C^{-1 / d_{2}} y_{0, A_{1}, A_{2}} & \geq \widetilde{\eta}_{2}-y_{2}\left(\tau_{2}, C^{-1 / d_{2}} x_{0}\right) \geq \widetilde{\eta}_{2}-\widetilde{y}_{2}\left(C^{-1 / d_{2}} x_{0}\right) \\
& \geq \widetilde{\eta}_{2}-\widetilde{y}_{2}\left(C_{2}^{-1 / d_{2}} x_{0}\right)=\text { const. }>0
\end{aligned}
$$

and therefore always the second case is valid.

CASE $2: \bar{y}<\widetilde{\eta}_{2}$. Choose $\left(x_{0} / \widetilde{x}_{2}(\bar{y})\right)^{d_{2}}$ and $\left(\widetilde{y}_{1}\left(x_{0}\right) / \bar{y}\right)^{d_{2}}$ as division points if they lie in $\left[C_{1}, C_{2}\right]$.

CASE 2.1: $C_{\mu}^{*} \leq\left(x_{0} / \widetilde{x}_{2}(\bar{y})\right)^{d_{2}}$. Then $C \leq\left(x_{0} / \widetilde{x}_{2}(\bar{y})\right)^{d_{2}}-A_{1}^{-\varepsilon}$ and by Taylor's theorem $C^{-1 / d_{2}} \geq \widetilde{x}_{2}(\bar{y}) / x_{0}+K_{40} A_{1}^{-\varepsilon}$ with some constant $K_{40}>0$. Applying Taylor's theorem again gives

$$
\widetilde{y}_{2}\left(C^{-1 / d_{2}} x_{0}\right) \leq \widetilde{y}_{2}\left(\widetilde{x}_{2}(\bar{y})+x_{0} K_{40} A_{1}^{-\varepsilon}\right) \leq \bar{y}-K_{41} A_{1}^{-\varepsilon}
$$

with some constant $K_{41}>0$ and so

$$
\begin{aligned}
\bar{y}-C^{-1 / d_{2}} y_{0, A_{1}, A_{2}} & \geq \bar{y}-y_{2}\left(\tau_{2}, C^{-1 / d_{2}} x_{0}\right) \geq \bar{y}-\widetilde{y}_{2}\left(C^{-1 / d_{2}} x_{0}\right) \\
& \geq K_{41} A_{1}^{-\varepsilon} \geq \tau_{1}^{\kappa}
\end{aligned}
$$

if $0<\varepsilon<\kappa / d_{1}$. Therefore always the second case is valid.

CASE 2.2: $C_{\mu-1}^{*} \geq\left(x_{0} / \widetilde{x}_{2}(\bar{y})\right)^{d_{2}}$.

CASE 2.2.1: $C_{\mu-1}^{*} \geq\left(\widetilde{y}_{1}\left(x_{0}\right) / \bar{y}\right)^{d_{2}}$. Then $C \geq\left(\widetilde{y}_{1}\left(x_{0}\right) / \bar{y}\right)^{d_{2}}+A_{1}^{-\varepsilon}$ and by Taylor's theorem $C^{1 / d_{2}} \geq \widetilde{y}_{1}\left(x_{0}\right) / \bar{y}+K_{40} A_{1}^{-\varepsilon}$. Consequently,

$$
\begin{aligned}
\bar{y}-C^{-1 / d_{2}} y_{0, A_{1}, A_{2}} & \geq \bar{y}-C^{-1 / d_{2}} y_{1}\left(\tau_{1}, x_{0}\right) \geq \bar{y}-C^{-1 / d_{2}} \widetilde{y}_{1}\left(x_{0}\right) \\
& \geq \bar{y} C^{-1 / d_{2}} K_{40} A_{1}^{-\varepsilon} \geq \tau_{1}^{\kappa}
\end{aligned}
$$

if $0<\varepsilon<\kappa / d_{1}$. Therefore always the second case is valid.

CASE 2.2.2: $C_{\mu}^{*} \leq\left(\widetilde{y}_{1}\left(x_{0}\right) / \bar{y}\right)^{d_{2}}$. Then $\left(x_{0} / \widetilde{x}_{2}(\bar{y})\right)^{d_{2}}+A_{1}^{-\varepsilon} \leq C \leq$ $\left(\widetilde{y}_{1}\left(x_{0}\right) / \bar{y}\right)^{d_{2}}-A_{1}^{-\varepsilon}$ and by Taylor's theorem

$$
C^{-1 / d_{2}} \leq \frac{\widetilde{x}_{2}(\bar{y})}{x_{0}}-K_{40} A_{1}^{-\varepsilon}, \quad C^{1 / d_{2}} \leq \frac{\widetilde{y}_{1}\left(x_{0}\right)}{\bar{y}}-K_{40} A_{1}^{-\varepsilon} .
$$

Applying Taylor's theorem again gives

$$
\widetilde{y}_{2}\left(C^{-1 / d_{2}} x_{0}\right) \geq \widetilde{y}_{2}\left(\widetilde{x}_{2}(\bar{y})-K_{40} x_{0} A_{1}^{-\varepsilon}\right) \geq \bar{y}+K_{41} A_{1}^{-\varepsilon} .
$$

With further constants $K_{42}, K_{43}>0$ it follows that

$$
\begin{aligned}
C^{-1 / d_{2}} y_{0, A_{1}, A_{2}} & =\min \left\{C^{-1 / d_{2}} y_{1}\left(\tau_{1}, x_{0}\right), y_{2}\left(\tau_{2}, C^{-1 / d_{2}} x_{0}\right)\right\} \\
& \geq \min \left\{C^{-1 / d_{2}}\left(\widetilde{y}_{1}\left(x_{0}\right)-K_{42} \tau_{1}\right), \widetilde{y}_{2}\left(C^{-1 / d_{2}} x_{0}\right)-K_{42} \tau_{2}\right\}
\end{aligned}
$$




$$
\begin{aligned}
& \geq \min \left\{\bar{y}+\bar{y} C^{-1 / d_{2}} K_{40} A_{1}^{-\varepsilon}-C^{-1 / d_{2}} K_{42} \tau_{1},\right. \\
& \left.\quad \bar{y}+K_{41} A_{1}^{-\varepsilon}-K_{42} \tau_{2}\right\} \\
& \geq \bar{y}+K_{43} A_{1}^{-\varepsilon} \geq \bar{y}+\tau_{1}^{\kappa}
\end{aligned}
$$

if $0<\varepsilon<\min \left\{1 / d_{1}, \kappa / d_{1}\right\}$ and so always the first case is valid.

LEMma 7.8. There is a decomposition $C_{1}=C_{0}^{*}<\ldots<C_{m}^{*}=C_{2}$ and constants $\varepsilon, K>0$ with the property: For $1 \leq \mu \leq m, 1 \leq \tau \leq t$ we have

$$
\left(C_{\mu-1}^{*}, C_{\mu}^{*}\right) \cap\left[c_{\tau}, d_{\tau}\right]=\emptyset \quad \text { or } \quad\left[C_{\mu-1}^{*}, C_{\mu}^{*}\right] \subseteq\left[c_{\tau}, d_{\tau}\right]
$$

In the latter case uniformly for $A_{1}, A_{2} \geq K$ with $C:=A_{2} A_{1}^{-d_{2} / d_{1}} \in$ $\left(C_{\mu-1}^{*}+A_{1}^{-\varepsilon}, C_{\mu}^{*}-A_{1}^{-\varepsilon}\right)$, one of the two cases $m_{\tau}(C) \leq y_{0, A_{1}, A_{2}}$ or $m_{\tau}(C) \geq$ $y_{0, A_{1}, A_{2}}$ is valid.

Pr o of. As decomposition points the following points are chosen if they lie in $\left[C_{1}, C_{2}\right]$ :

(1) $h\left(x_{0}\right)$ and $c_{\tau}, d_{\tau}$ for $1 \leq \tau \leq t$.

(2) The decomposition points arising from Lemma 7.6 applied to $\bar{y}=\eta_{\tau}$, $1 \leq \tau \leq t$.

Let $1 \leq \mu \leq m, 1 \leq \tau \leq t$. Then $\left(C_{\mu-1}^{*}, C_{\mu}^{*}\right) \cap\left[c_{\tau}, d_{\tau}\right]=\emptyset$ or $\left[C_{\mu-1}^{*}, C_{\mu}^{*}\right] \subseteq$ $\left[c_{\tau}, d_{\tau}\right]$. Assume the latter case. Then uniformly for $A_{1}, A_{2} \geq K$ with $C:=A_{2} A_{1}^{-d_{2} / d_{1}} \in\left(C_{\mu-1}^{*}+A_{1}^{-\varepsilon}, C_{\mu}^{*}-A_{1}^{-\varepsilon}\right)$ one of the three cases $y_{0, A_{1}, A_{2}}$ $\leq \eta_{\tau-1}, y_{0, A_{1}, A_{2}} \in\left(\eta_{\tau-1}, \eta_{\tau}\right]$ or $y_{0, A_{1}, A_{2}}>\eta_{\tau}$ is valid. In the first case $y_{0, A_{1}, A_{2}} \leq m_{\tau}(C)$. In the last case $y_{0, A_{1}, A_{2}}>m_{\tau}(C)$. For the remainder of the proof assume that the second case is valid. From the definition of $m_{\tau}$ it follows that

$$
m_{\tau}(C) \leq y_{0, A_{1}, A_{2}} \Leftrightarrow \begin{cases}C \leq l\left(y_{0, A_{1}, A_{2}}\right), & l \uparrow\left[\eta_{\tau-1}, \eta_{\tau}\right] \text { increasing } \\ C \geq l\left(y_{0, A_{1}, A_{2}}\right), & l \uparrow\left[\eta_{\tau-1}, \eta_{\tau}\right] \text { decreasing. }\end{cases}
$$

From (2.10) it follows that

$$
\left|\widetilde{y}_{1}\left(x_{0}\right)-C^{1 / d_{2}} \widetilde{y}_{2}\left(C^{-1 / d_{2}} x_{0}\right)\right| \gg\left|h\left(x_{0}\right)-C\right| \geq A_{1}^{-\varepsilon} .
$$

CASE 1: $C_{\mu-1}^{*} \geq h\left(x_{0}\right)$. Then $\widetilde{y}_{1}\left(x_{0}\right)<C^{1 / d_{2}} \widetilde{y}_{2}\left(C^{-1 / d_{2}} x_{0}\right)$ by $(2.16)$, and with (7.10) this gives $\widetilde{y}_{1}\left(x_{0}\right) \leq C^{1 / d_{2}} \widetilde{y}_{2}\left(C^{-1 / d_{2}} x_{0}\right)-K_{50} A_{1}^{-\varepsilon}$ with some constant $K_{50}>0$. If $0<\varepsilon<1 / d_{1}$ this gives

$$
\begin{aligned}
y_{1}\left(\tau_{1}, x_{0}\right) & =\widetilde{y}_{1}\left(x_{0}\right)+O\left(\tau_{1}\right) \\
& \leq C^{1 / d_{2}} y_{2}\left(\tau_{2}, C^{-1 / d_{2}} x_{0}\right)=C^{1 / d_{2}} \widetilde{y}_{2}\left(C^{-1 / d_{2}} x_{0}\right)+O\left(\tau_{1}\right)
\end{aligned}
$$

and consequently $y_{0}=y_{1}\left(\tau_{1}, x_{0}\right)=\widetilde{y}_{1}\left(x_{0}\right)+O\left(\tau_{1}\right)$. Therefore 


$$
\begin{aligned}
l\left(y_{0}\right) & =\widetilde{g}_{2}\left(\widetilde{x}_{1}\left(y_{0}\right), y_{0}\right)=\widetilde{g}_{2}\left(x_{0}+O\left(\tau_{1}\right), \widetilde{y}_{1}\left(x_{0}\right)+O\left(\tau_{1}\right)\right) \\
& =\widetilde{g}_{2}\left(x_{0}, \widetilde{y}_{1}\left(x_{0}\right)\right)+O\left(\tau_{1}\right) \\
& =h\left(x_{0}\right)+O\left(\tau_{1}\right) \leq C_{\mu-1}^{*}+O\left(\tau_{1}\right) \\
& \leq C-A_{1}^{-\varepsilon}+O\left(\tau_{1}\right)<C .
\end{aligned}
$$

Case 2: $C_{\mu}^{*} \leq h\left(x_{0}\right)$. From (2.16) and (7.10) it follows that

$$
\widetilde{y}_{1}\left(x_{0}\right) \geq C^{1 / d_{2}} \widetilde{y}_{2}\left(C^{-1 / d_{2}} x_{0}\right)+K_{50} A_{1}^{-\varepsilon} \text {. }
$$

Just as above,

$$
y_{0, A_{1}, A_{2}}=C^{1 / d_{2}} y_{2}\left(\tau_{2}, C^{-1 / d_{2}} x_{0}\right)=C^{1 / d_{2}} \widetilde{y}_{2}\left(C^{-1 / d_{2}} x_{0}\right)+O\left(\tau_{1}\right) .
$$

Taylor's theorem gives $\widetilde{x}_{1}\left(\widetilde{y}_{1}\left(x_{0}\right)-K_{50} A_{1}^{-\varepsilon}\right) \geq x_{0}+K_{51} A_{1}^{-\varepsilon}$ with some constant $K_{51}>0$. From (7.11) it follows that

$$
\begin{aligned}
l\left(y_{0}\right)= & \widetilde{g}_{2}\left(\widetilde{x}_{1}\left(y_{0}\right), y_{0}\right) \\
= & \widetilde{g}_{2}\left(\widetilde{x}_{1}\left(C^{1 / d_{2}} \widetilde{y}_{2}\left(C^{-1 / d_{2}} x_{0}\right)\right)+O\left(\tau_{1}\right), C^{1 / d_{2}} \widetilde{y}_{2}\left(C^{-1 / d_{2}} x_{0}\right)+O\left(\tau_{1}\right)\right) \\
\geq & \widetilde{g}_{2}\left(\widetilde{x}_{1}\left(\widetilde{y}_{1}\left(x_{0}\right)-K_{50} A_{1}^{-\varepsilon}\right), C^{1 / d_{2}} \widetilde{y}_{2}\left(C^{-1 / d_{2}} x_{0}\right)\right)+O\left(\tau_{1}\right) \\
\geq & \widetilde{g}_{2}\left(x_{0}+K_{51} A_{1}^{-\varepsilon}, C^{1 / d_{2}} \widetilde{y}_{2}\left(C^{-1 / d_{2}} x_{0}\right)\right)+O\left(\tau_{1}\right) \\
\geq & \widetilde{g}_{2}\left(x_{0}, C^{1 / d_{2}} \widetilde{y}_{2}\left(C^{-1 / d_{2}} x_{0}\right)\right)+a_{d_{2} 0}^{(2)}\left(x_{0}+K_{51} A_{1}^{-\varepsilon}\right)^{d_{2}}-a_{d_{2} 0}^{(2)} x_{0}^{d_{2}} \\
& +O\left(\tau_{1}\right) \\
\geq & C+a_{d_{2} 0}^{(2)} d_{2} x_{0}^{d_{2}-1} K_{51} A_{1}^{-\varepsilon}+O\left(\tau_{1}\right)>C .
\end{aligned}
$$

(7.9) shows in Cases 1 and 2 that uniformly throughout the given range of $\left(A_{1}, A_{2}\right)$ one of the two cases $m_{\tau}(C) \leq y_{0}$ or $m_{\tau}(C) \geq y_{0}$ is valid.

Choose $Z \subseteq \mathbb{R}_{0}^{+},|Z|<\infty$ with the properties:

$\left(\mathrm{Z}^{\#} 1\right) \quad$ The zeros of $\widetilde{x}_{j}^{\prime \prime} \widetilde{x}_{j}^{\prime \prime \prime}$ in $\left[0, \widetilde{\eta}_{j}\right]$ are contained in $Z$ for $j=1,2$.

$\left(\mathrm{Z}^{\#} 2\right) \quad$ The zeros of $\widetilde{y}_{j}^{\prime \prime} \widetilde{y}_{j}^{\prime \prime \prime}$ in $\left[0, \widetilde{\xi}_{j}\right]$ are contained in $\widetilde{x}_{j}(Z)$ for $j=1,2$.

$\left(\mathrm{Z}^{\#} 3\right) \quad \eta_{0}, \ldots, \eta_{t}, y_{1}, y_{2}, h\left(x_{0}\right) \in Z$.

$\left(\mathrm{Z}^{\#} 4\right) \quad$ If $\widetilde{x}_{j}^{\prime}(0)=0 \neq \widetilde{x}_{j}^{\prime \prime}(0)$ for $j=1$ or $j=2$, then the value of $\bar{\xi}$ which comes from the application of Proposition 6.1 is contained in $\widetilde{x}_{j}(Z)$.

Choose $0=\zeta_{0}<\ldots<\zeta_{n}$ with the properties:

$\left(\mathrm{Z}^{\#} 5\right) \quad Z \subseteq\left\{\zeta_{0}, \ldots, \zeta_{n}\right\}$.

$\left(\mathrm{Z}^{\#} 6\right) \quad$ For each $\zeta, \zeta^{\prime} \in Z$ with $\zeta<\zeta^{\prime}$ there is some $1 \leq \nu \leq n$ with $\zeta<\zeta_{\nu}<\zeta^{\prime}$.

For $1 \leq \tau \leq t$ define $\left[c_{\tau}^{\prime}, d_{\tau}^{\prime}\right]:=m_{\tau}^{-1}\left(\left(-\infty, y_{1}\right]\right)$ and $\widetilde{m}_{\tau}(C):=C^{-1 / d_{2}} m_{\tau}(C)$ on $\left[c_{\tau}^{\prime}, d_{\tau}^{\prime}\right]$. Now choose a decomposition $C_{1}=C_{0}^{*}<\ldots<C_{m}^{*}=C_{2}$ and constant $\varepsilon>0$ with the properties: 
$\left(\mathrm{C}^{\#} 1\right) \quad$ If $c_{\tau}^{\prime} \in\left[C_{1}, C_{2}\right]$ for some $1 \leq \tau \leq t$ then $c_{\tau}^{\prime} \in\left\{C_{0}^{*}, \ldots, C_{m}^{*}\right\}$. The same holds for $c_{\tau}, d_{\tau}^{\prime}$ and $d_{\tau}$.

$\left(\mathrm{C}^{\#} 2\right) \quad$ For $1 \leq \tau \leq t, 1 \leq \mu \leq m, 0 \leq \nu \leq n$ with $\left[C_{\mu-1}^{*}, C_{\mu}^{*}\right] \subseteq\left[c_{\tau}^{\prime}, d_{\tau}^{\prime}\right]$ : For $A_{1}, A_{2} \geq K$ with $C:=A_{2} A_{1}^{-d_{2} / d_{1}} \in\left(C_{\mu-1}^{*}+A_{1}^{-\varepsilon}, C_{\mu}^{*}-A_{1}^{-\varepsilon}\right)$ we have

$$
\left|m_{\tau}(C)-\zeta_{\nu}\right| \geq \tau_{1}^{\kappa}, \quad\left|\widetilde{m}_{\tau}(C)-\zeta_{\nu}\right| \geq \tau_{2}^{\kappa} .
$$

$\left(\mathrm{C}^{\#} 3\right) \quad$ For $1 \leq \mu \leq m, 1 \leq \nu^{\prime} \leq n, 0 \leq \nu \leq n$ : For $A_{1}, A_{2} \geq K$ with $C:=A_{2} A_{1}^{-d_{2} / d_{1}} \in\left(C_{\mu-1}^{*}+A_{1}^{-\varepsilon}, C_{\mu}^{*}-A_{1}^{-\varepsilon}\right)$ we have

$$
\left|C^{-1 / d_{2}} \zeta_{\nu^{\prime}}-\zeta_{\nu}\right| \geq \tau_{2}^{\kappa} \text {. }
$$

$\left(\mathrm{C}^{\#} 4\right) \quad$ For $1 \leq \tau \leq t, 1 \leq \mu \leq m, 1 \leq \nu^{\prime} \leq n, 0 \leq \nu \leq n$ we have $\zeta_{\nu} \notin m_{\tau}\left(\left(C_{\mu-1}^{*}, C_{\mu}^{*}\right) \cap\left[c_{\tau}^{\prime}, d_{\tau}^{\prime}\right]\right), \quad \zeta_{\nu} \notin \widetilde{m}_{\tau}\left(\left(C_{\mu-1}^{*}, C_{\mu}^{*}\right) \cap\left[c_{\tau}^{\prime}, d_{\tau}^{\prime}\right]\right)$, $\zeta_{\nu} \neq C^{-1 / d_{2}} \zeta_{\nu^{\prime}} \quad$ for $C \in\left(C_{\mu-1}^{*}, C_{\mu}^{*}\right)$.

$\left(\mathrm{C}^{\#} 5\right) \quad$ All the decomposition points which arise from the application of Lemmas 7.6 and 7.7 to $\bar{y}=\zeta_{1}, \ldots, \zeta_{n}$ and from Lemma 7.8 are contained in $\left\{C_{0}^{*}, \ldots, C_{m}^{*}\right\}$.

The following lemma is the analogue of Lemma 7.4.

Lemma 7.9. Let $1 \leq \mu \leq m, 1 \leq \tau \leq t$. Then uniformly for $A_{1}, A_{2} \geq K$ with $C:=A_{2} A_{1}^{-d_{2} / d_{1}} \in\left(C_{\mu-1}^{*}+A_{1}^{-\varepsilon}, C_{\mu}^{*}-A_{1}^{-\varepsilon}\right)$ one of the two cases is valid:

(1) $y_{0, A_{1}, A_{2}} \leq \eta_{\tau-1}$ and $R_{\tau}^{\#}\left(A_{1}, A_{2}\right)=0$.

(2) $y_{0, A_{1}, A_{2}} \geq \eta_{\tau-1}$ and

$$
\begin{aligned}
R_{\tau}^{\#}\left(A_{1}, A_{2}\right)= & \int_{A_{1}^{1 / d_{1}} \eta_{\tau-1}}^{A_{1}^{1 / d_{1}} \min \left\{y_{0}, \eta_{\tau}\right\}} f_{A_{1}, A_{2}}^{-1}(y) d y-\frac{1}{2} A_{1}^{1 / d_{1}}\left(\min \left\{\eta_{\tau}, y_{0}\right\}-\eta_{\tau-1}\right) \\
& +T_{1}^{\#(\mu, \varrho)}\left(A_{1}\right)+T_{2}^{\#(\mu, \varrho)}\left(A_{2}\right)+U_{1}^{\#(\mu, \varrho)}\left(A_{1}\right)+U_{2}^{\#(\mu, \varrho)}\left(A_{2}\right) \\
& +\psi\left(A_{1}^{1 / d_{1}} \eta_{\tau-1}\right) f_{A_{1}, A_{2}}^{-1}\left(A_{1}^{1 / d_{1}} \eta_{\tau-1}\right) \\
& -\psi\left(A_{1}^{1 / d_{1}} \min \left\{\eta_{\tau}, y_{0}\right\}\right) f_{A_{1}, A_{2}}^{-1}\left(A_{1}^{1 / d_{1}} \min \left\{\eta_{\tau}, y_{0}\right\}\right) \\
& +O\left(A_{1}^{46 /\left(73 d_{1}\right)}\left(\log A_{1}\right)^{315 / 146}\right) .
\end{aligned}
$$

Pr o of. Again several cases have to be distinguished. From Lemma 7.6 for $\bar{y}=\eta_{\tau-1}$ (in case $\tau \geq 2$ ) it follows that always $y_{0} \leq \eta_{\tau-1}$ or $y_{0}>\eta_{\tau-1}$. Assume the latter case.

CASE 1: $\left[C_{\mu-1}^{*}, C_{\mu}^{*}\right] \subseteq\left[c_{\tau}^{\prime}, d_{\tau}^{\prime}\right]$. From Lemma 7.6 for $\bar{y}=\eta_{\tau}$ and Lemma 7.8 it follows that uniformly in the given range of $\left(A_{1}, A_{2}\right)$ one of the two cases $y_{0} \leq \eta_{\tau}$ or $y_{0}>\eta_{\tau}$ and one of the two cases $m_{\tau}(C) \leq y_{0}$ or $m_{\tau}(C) \geq y_{0}$ is valid. 
CASE 1.1: $m_{\tau}(C) \leq y_{0} \leq \eta_{\tau}$. Applying Lemma 3.1 with $x, y$ interchanged gives the decomposition in the following two cases.

CASE 1.1.1: If $l \uparrow\left[c_{\tau}, d_{\tau}\right]$ increases then

$$
\begin{aligned}
R_{\tau}^{\#}= & \#\left\{(x, y) \in \mathbb{Z}^{2} \mid A_{1}^{1 / d_{1}} \eta_{\tau-1}<y \leq A_{1}^{1 / d_{1}} m_{\tau}(C),\right. \\
& \left.0<x \leq A_{1}^{1 / d_{1}} x_{1}\left(\tau_{1}, \tau_{1} y\right)\right\} \\
+ & \#\left\{(x, y) \in \mathbb{Z}^{2} \mid A_{1}^{1 / d_{1}} m_{\tau}(C)<y \leq A_{1}^{1 / d_{1}} y_{0},\right. \\
& \left.0<x \leq A_{2}^{1 / d_{2}} x_{2}\left(\tau_{2}, \tau_{2} y\right)\right\} \\
& +O\left(A_{1}^{46 /\left(73 d_{1}\right)}\right) .
\end{aligned}
$$

CASE 1.1.2: If $l\left\lceil\left[c_{\tau}, d_{\tau}\right]\right.$ decreases then

$$
\begin{aligned}
R_{\tau}^{\#}= & \#\left\{(x, y) \in \mathbb{Z}^{2} \mid A_{1}^{1 / d_{1}} \eta_{\tau-1}<y \leq A_{1}^{1 / d_{1}} m_{\tau}(C),\right. \\
& \left.0<x \leq A_{2}^{1 / d_{2}} x_{2}\left(\tau_{2}, \tau_{2} y\right)\right\} \\
+ & \#\left\{(x, y) \in \mathbb{Z}^{2} \mid A_{1}^{1 / d_{1}} m_{\tau}(C)<y \leq A_{1}^{1 / d_{1}} y_{0},\right. \\
& \left.0<x \leq A_{1}^{1 / d_{1}} x_{1}\left(\tau_{1}, \tau_{1} y\right)\right\} \\
& +O\left(A_{1}^{46 /\left(73 d_{1}\right)}\right) .
\end{aligned}
$$

CASE 1.2: $y_{0} \leq m_{\tau}(C) \leq \eta_{\tau}$.

CASE 1.2.1: If $l \uparrow\left[c_{\tau}, d_{\tau}\right]$ increases then

$$
\begin{aligned}
R_{\tau}^{\#}= & \#\left\{(x, y) \in \mathbb{Z}^{2} \mid A_{1}^{1 / d_{1}} \eta_{\tau-1}<y \leq A_{1}^{1 / d_{1}} y_{0}, 0<x \leq A_{1}^{1 / d_{1}} x_{1}\left(\tau_{1}, \tau_{1} y\right)\right\} \\
& +O\left(A_{1}^{46 /\left(73 d_{1}\right)}\right) .
\end{aligned}
$$

CASE 1.2.2: If $l \uparrow\left[c_{\tau}, d_{\tau}\right]$ decreases then

$$
\begin{aligned}
R_{\tau}^{\#}= & \#\left\{(x, y) \in \mathbb{Z}^{2} \mid A_{1}^{1 / d_{1}} \eta_{\tau-1}<y \leq A_{1}^{1 / d_{1}} y_{0}, 0<x \leq A_{2}^{1 / d_{2}} x_{2}\left(\tau_{2}, \tau_{2} y\right)\right\} \\
& +O\left(A_{1}^{46 /\left(73 d_{1}\right)}\right) .
\end{aligned}
$$

CASE 1.3: $y_{0}>\eta_{\tau}, y_{0} \geq m_{\tau}(C)$.

CASE 1.3.1: If $l \uparrow\left[c_{\tau}, d_{\tau}\right]$ increases then

$$
\begin{aligned}
R_{\tau}^{\#}= & \#\left\{(x, y) \in \mathbb{Z}^{2} \mid A_{1}^{1 / d_{1}} \eta_{\tau-1}<y \leq A_{1}^{1 / d_{1}} m_{\tau}(C),\right. \\
& \left.0<x \leq A_{1}^{1 / d_{1}} x_{1}\left(\tau_{1}, \tau_{1} y\right)\right\} \\
+ & \#\left\{(x, y) \in \mathbb{Z}^{2} \mid A_{1}^{1 / d_{1}} m_{\tau}(C)<y \leq A_{1}^{1 / d_{1}} \eta_{\tau},\right. \\
& \left.0<x \leq A_{2}^{1 / d_{2}} x_{2}\left(\tau_{2}, \tau_{2} y\right)\right\} \\
+ & O\left(A_{1}^{46 /\left(73 d_{1}\right)}\right) .
\end{aligned}
$$

CASE 1.3.2: If $l \uparrow\left[c_{\tau}, d_{\tau}\right]$ decreases then

$$
\begin{aligned}
R_{\tau}^{\#}=\#\left\{(x, y) \in \mathbb{Z}^{2} \mid A_{1}^{1 / d_{1}} \eta_{\tau-1}<y \leq A_{1}^{1 / d_{1}} m_{\tau}(C),\right. \\
\left.0<x \leq A_{2}^{1 / d_{2}} x_{2}\left(\tau_{2}, \tau_{2} y\right)\right\}
\end{aligned}
$$




$$
\begin{aligned}
& +\#\left\{(x, y) \in \mathbb{Z}^{2} \mid A_{1}^{1 / d_{1}} m_{\tau}(C)<y \leq A_{1}^{1 / d_{1}} \eta_{\tau},\right. \\
& \left.\quad 0<x \leq A_{1}^{1 / d_{1}} x_{1}\left(\tau_{1}, \tau_{1} y\right)\right\} \\
& +O\left(A_{1}^{46 /\left(73 d_{1}\right)}\right) .
\end{aligned}
$$

The fourth combination $m_{\tau}(C) \geq y_{0}>\eta_{\tau}$ is not possible because always $m_{\tau}(C) \leq \eta_{\tau}$.

CASE 2: $\left[C_{\mu-1}^{*}, C_{\mu}^{*}\right] \subseteq\left[c_{\tau}, c_{\tau}^{\prime}\right]$ or $\subseteq\left[d_{\tau}^{\prime}, d_{\tau}\right]$. Then $y_{0} \leq y_{1}<m_{\tau}(C) \leq \eta_{\tau}$.

CASE 2.1: If $l \uparrow\left[c_{\tau}, d_{\tau}\right]$ increases then

$$
\begin{aligned}
R_{\tau}^{\#}= & \#\left\{(x, y) \in \mathbb{Z}^{2} \mid A_{1}^{1 / d_{1}} \eta_{\tau-1}<y \leq A_{1}^{1 / d_{1}} y_{0}, 0<x \leq A_{1}^{1 / d_{1}} x_{1}\left(\tau_{1}, \tau_{1} y\right)\right\} \\
& +O\left(A_{1}^{46 /\left(73 d_{1}\right)}\right) .
\end{aligned}
$$

CASE 2.2: If $l \uparrow\left[c_{\tau}, d_{\tau}\right]$ decreases then

$$
\begin{aligned}
R_{\tau}^{\#}= & \#\left\{(x, y) \in \mathbb{Z}^{2} \mid A_{1}^{1 / d_{1}} \eta_{\tau-1}<y \leq A_{1}^{1 / d_{1}} y_{0}, 0<x \leq A_{2}^{1 / d_{2}} x_{2}\left(\tau_{2}, \tau_{2} y\right)\right\} \\
& +O\left(A_{1}^{46 /\left(73 d_{1}\right)}\right) .
\end{aligned}
$$

CASE 3: $C_{\mu}^{*} \leq c_{\tau}$. From Lemma 7.6 for $\bar{y}=\eta_{\tau}$ it follows that always $y_{0} \leq \eta_{\tau}$ or $y_{0}>\eta_{\tau}$.

CASE 3.1: If $y_{0} \leq \eta_{\tau}$ then

$R_{\tau}^{\#}=\#\left\{(x, y) \in \mathbb{Z}^{2} \mid A_{1}^{1 / d_{1}} \eta_{\tau-1}<y \leq A_{1}^{1 / d_{1}} y_{0}, 0<x \leq A_{2}^{1 / d_{2}} x_{2}\left(\tau_{2}, \tau_{2} y\right)\right\}$.

CASE 3.2: If $y_{0}>\eta_{\tau}$ then

$R_{\tau}^{\#}=\#\left\{(x, y) \in \mathbb{Z}^{2} \mid A_{1}^{1 / d_{1}} \eta_{\tau-1}<y \leq A_{1}^{1 / d_{1}} \eta_{\tau}, 0<x \leq A_{2}^{1 / d_{2}} x_{2}\left(\tau_{2}, \tau_{2} y\right)\right\}$.

CASE 4: $C_{\mu-1}^{*} \geq d_{\tau}$.

CASE 4.1: If $y_{0} \leq \eta_{\tau}$ then

$R_{\tau}^{\#}=\#\left\{(x, y) \in \mathbb{Z}^{2} \mid A_{1}^{1 / d_{1}} \eta_{\tau-1}<y \leq A_{1}^{1 / d_{1}} y_{0}, 0<x \leq A_{1}^{1 / d_{1}} x_{1}\left(\tau_{1}, \tau_{1} y\right)\right\}$.

CASE 4.2 : If $y_{0}>\eta_{\tau}$ then

$R_{\tau}^{\#}=\#\left\{(x, y) \in \mathbb{Z}^{2} \mid A_{1}^{1 / d_{1}} \eta_{\tau-1}<y \leq A_{1}^{1 / d_{1}} \eta_{\tau}, 0<x \leq A_{1}^{1 / d_{1}} x_{1}\left(\tau_{1}, \tau_{1} y\right)\right\}$.

As in the proof of Lemma 7.4 only Cases 1.1.1 and 1.1.2 are pursued further. The other cases are similar but somewhat easier.

In Case 1.1.1 use $\left(\mathrm{C}^{\#} 4\right),\left(\mathrm{C}^{\#} 5\right),(2.8)$ and $\left(\mathrm{Z}^{\#} 3\right)$ and choose $1 \leq \nu_{j} \leq n$ with the properties

$$
\begin{gathered}
m_{\tau}\left(\left(C_{\mu-1}^{*}, C_{\mu}^{*}\right)\right) \subseteq\left(\zeta_{\nu_{0}-1}, \zeta_{\nu_{0}}\right) \subseteq\left(\eta_{\tau-1}, \min \left\{\eta_{\tau}, y_{1}\right\}\right), \quad \zeta_{\nu_{1}-1}=\eta_{\tau-1}, \\
\zeta_{\nu_{2}}=\min \left\{\eta_{\tau}, y_{1}\right\}, \quad \nu_{1} \leq \nu_{0} \leq \nu_{2}, \quad \zeta_{\nu_{2}}<\widetilde{\eta}_{1} \\
\widetilde{m}_{\tau}\left(\left(C_{\mu-1}^{*}, C_{\mu}^{*}\right)\right) \subseteq\left(\zeta_{\nu_{3}-1}, \zeta_{\nu_{3}}\right), \\
C^{-1 / d_{2}} y_{0, A_{1}, A_{2}} \in\left(\zeta_{\nu_{4}-1}, \zeta_{\nu_{4}}\right) \quad \text { for } A_{1}, A_{2} \geq K
\end{gathered}
$$


with

$$
C:=A_{2} A_{1}^{-d_{2} / d_{1}} \in\left(C_{\mu-1}^{*}+A_{1}^{-\varepsilon}, C_{\mu}^{*}-A_{1}^{-\varepsilon}\right), \quad \nu_{3} \leq \nu_{4}, \quad \zeta_{\nu_{4}}<\widetilde{\eta}_{2} .
$$

Then

$$
\begin{aligned}
& R_{\tau}^{\#}=\sum_{\nu=\nu_{1}}^{\nu_{0}-1} \#\left\{(x, y) \in \mathbb{Z}^{2} \mid A_{1}^{1 / d_{1}} \zeta_{\nu-1}<y \leq A_{1}^{1 / d_{1}} \zeta_{\nu},\right. \\
& \left.0<x \leq A_{1}^{1 / d_{1}} x_{1}\left(\tau_{1}, \tau_{1} y\right)\right\} \\
& +\#\left\{(x, y) \in \mathbb{Z}^{2} \mid A_{1}^{1 / d_{1}} \zeta_{\nu_{0}-1}<y \leq A_{1}^{1 / d_{1}} m_{\tau}(C),\right. \\
& \left.0<x \leq A_{1}^{1 / d_{1}} x_{1}\left(\tau_{1}, \tau_{1} y\right)\right\}
\end{aligned}
$$

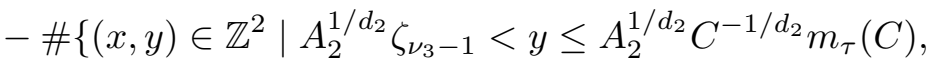

$$
\begin{aligned}
& \left.0<x \leq A_{2}^{1 / d_{2}} x_{2}\left(\tau_{2}, \tau_{2} y\right)\right\} \\
& +\sum_{\nu=\nu_{3}}^{\nu_{4}-1} \#\left\{(x, y) \in \mathbb{Z}^{2} \mid A_{2}^{1 / d_{2}} \zeta_{\nu-1}<y \leq A_{2}^{1 / d_{2}} \zeta_{\nu},\right. \\
& \left.0<x \leq A_{2}^{1 / d_{2}} x_{2}\left(\tau_{2}, \tau_{2} y\right)\right\} \\
& +\#\left\{(x, y) \in \mathbb{Z}^{2} \mid A_{2}^{1 / d_{2}} \zeta_{\nu_{4}-1}<y \leq A_{2}^{1 / d_{2}} C^{-1 / d_{2}} y_{0},\right. \\
& \left.0<x \leq A_{2}^{1 / d_{2}} x_{2}\left(\tau_{2}, \tau_{2} y\right)\right\} \\
& +O\left(A_{1}^{46 /\left(73 d_{1}\right)}\right) \text {. }
\end{aligned}
$$

In Case 1.1.2 choose $1 \leq \nu_{j} \leq n$ with the properties

$$
\begin{gathered}
m_{\tau}\left(\left(C_{\mu-1}^{*}, C_{\mu}^{*}\right)\right) \subseteq\left(\zeta_{\nu_{0}-1}, \zeta_{\nu_{0}}\right) \subseteq\left(\eta_{\tau-1}, \min \left\{\eta_{\tau}, y_{1}\right\}\right), \quad \zeta_{\nu_{1}-1}=\eta_{\tau-1}, \\
\zeta_{\nu_{2}}=\min \left\{\eta_{\tau}, y_{1}\right\}, \quad \nu_{1} \leq \nu_{0} \leq \nu_{2}, \quad \zeta_{\nu_{2}}<\widetilde{\eta}_{1}, \\
\tilde{m}_{\tau}\left(\left(C_{\mu-1}^{*}, C_{\mu}^{*}\right)\right) \subseteq\left(\zeta_{\nu_{3}-1}, \zeta_{\nu_{3}}\right) \subseteq\left[0, y_{2}\right]
\end{gathered}
$$

$\zeta_{\nu_{3}}<\widetilde{\eta}_{2}, \quad C^{-1 / d_{2}} \widetilde{\eta}_{\tau-1} \in\left(\zeta_{\nu_{4}-1}, \zeta_{\nu_{4}}\right)$ or $\equiv \zeta_{\nu_{4}-1}($ if $\tau=1), \quad \nu_{4} \leq \nu_{3}$,

$$
y_{0, A_{1}, A_{2}} \in\left(\zeta_{\nu_{5}-1}, \zeta_{\nu_{5}}\right] \quad \text { for } A_{1}, A_{2} \geq K
$$

with

$$
C:=A_{2} A_{1}^{-d_{2} / d_{1}} \in\left(C_{\mu-1}^{*}+A_{1}^{-\varepsilon}, C_{\mu}^{*}-A_{1}^{-\varepsilon}\right), \quad \nu_{0} \leq \nu_{5} .
$$

Then

$$
\begin{aligned}
& R_{\tau}^{\#}=-\#\left\{(x, y) \in \mathbb{Z}^{2} \mid A_{2}^{1 / d_{2}} \zeta_{\nu_{4}-1}<y \leq A_{2}^{1 / d_{2}} C^{-1 / d_{2}} \eta_{\tau-1},\right. \\
&\left.0<x \leq A_{2}^{1 / d_{2}} x_{2}\left(\tau_{2}, \tau_{2} y\right)\right\} \\
&+\sum_{\nu=\nu_{4}}^{\nu_{3}-1} \#\left\{(x, y) \in \mathbb{Z}^{2} \mid A_{2}^{1 / d_{2}} \zeta_{\nu-1}<y \leq A_{2}^{1 / d_{2}} \zeta_{\nu},\right. \\
&+\#\left\{(x, y) \in \mathbb{Z}^{2} \mid A_{2}^{1 / d_{2}} \zeta_{\nu_{3}-1}<y \leq A_{2}^{1 / d_{2}} C^{-1 / d_{2}} m_{\tau}(C),\right. \\
&\left.0<x \leq A_{2}^{1 / d_{2}} x_{2}\left(\tau_{2}, \tau_{2} y\right)\right\}
\end{aligned}
$$




$$
\begin{aligned}
& -\#\left\{(x, y) \in \mathbb{Z}^{2} \mid A_{1}^{1 / d_{1}} \zeta_{\nu_{0}-1}<y \leq A_{1}^{1 / d_{1}} m_{\tau}(C),\right. \\
& +\sum_{\nu=\nu_{0}} \#\left\{(x, y) \in \mathbb{Z}^{2} \mid A_{1}^{1 / d_{1}} \zeta_{\nu-1}<y \leq A_{1}^{1 / d_{1}} \zeta_{\nu},\right. \\
& +\#\left\{(x, y) \in \mathbb{Z}^{2} \mid A_{1}^{1 / d_{1}} x_{1}\left(\tau_{1}, \tau_{1} y\right)\right\} \\
& \left.+0<x \leq A_{1}^{1 / d_{1}} x_{1}\left(\tau_{1}, \tau_{1} y\right)\right\} \\
& +O\left(A_{1}^{46 /\left(73 d_{1}\right)}\right) . \\
& \quad 0<A_{1}^{1 / d_{1}} y_{0}, \quad
\end{aligned}
$$

It follows from $\left(\mathrm{C}^{\#} 2\right)$ and $\left(\mathrm{C}^{\#} 3\right)$ that in each but the last set the endpoints of the respective intervals of $y$ when renormalized with the corresponding $A_{j}^{-1 / d_{j}}$ have a distance $\geq \tau_{j}^{\kappa}$ from all $\zeta_{\nu}$ with $0 \leq \nu \leq n$ or are equal to one of these $\zeta_{\nu}$. From $\left(\mathrm{C}^{\#} 5\right)$ and Lemma 7.6 applied to $\bar{y}=\zeta_{\nu_{5}-1}$ (if $\nu_{5}>1$ ) and $\bar{y}=\zeta_{\nu_{5}}$ it follows that for the last set the same holds or that $\left|y_{0}-\zeta_{\nu_{5}}\right| \ll \tau_{1}$ or $\left|y_{0}-\zeta_{\nu_{5}-1}\right| \ll \tau_{1}$ uniformly in $\left(A_{1}, A_{2}\right)$. In the last case apply the trivial estimation to the last set.

The remainder of the proof is as in the proof of Lemma 7.4.

Corollary 7.10. Let $1 \leq \mu \leq m$. Then for $A_{1}, A_{2} \geq K$ with $C:=$ $A_{2} A_{1}^{-d_{2} / d_{1}} \in\left(C_{\mu-1}^{*}+A_{1}^{-\varepsilon}, C_{\mu}^{*}-A_{1}^{-\varepsilon}\right)$ we have

$$
\begin{aligned}
R^{\#}\left(A_{1}, A_{2}\right)= & \int_{0}^{A_{1}^{1 / d_{1}} y_{0}} f_{A_{1}, A_{2}}^{-1}(y) d y-\frac{1}{2} A_{1}^{1 / d_{1}} y_{0}+T_{1}^{\#(\mu)}\left(A_{1}\right)+T_{2}^{\#(\mu)}\left(A_{2}\right) \\
& +U_{1}^{\#(\mu)}\left(A_{1}\right)+U_{2}^{\#(\mu)}\left(A_{2}\right)-\frac{1}{2} f_{A_{1}, A_{2}}^{-1}(0) \\
& -\psi\left(A_{1}^{1 / d_{1}} y_{0}\right) f_{A_{1}, A_{2}}^{-1}\left(A_{1}^{1 / d_{1}} y_{0}\right) \\
& +O\left(A_{1}^{46 /\left(73 d_{1}\right)}\left(\log A_{1}\right)^{315 / 146}\right) .
\end{aligned}
$$

Corollaries 7.5 and 7.10 and (3.1) together with

$$
\begin{aligned}
& f_{A_{1}, A_{2}}(0)=\min \left\{A_{1}^{1 / d_{1}} \widetilde{\eta}_{1}, A_{2}^{1 / d_{2}} \widetilde{\eta}_{2}\right\}+O(1), \\
& f_{A_{1}, A_{2}}^{-1}(0)=\varrho_{A_{1}, A_{2}}=\min \left\{A_{1}^{1 / d_{1}} \widetilde{\xi}_{1}, A_{2}^{1 / d_{2}} \widetilde{\xi}_{2}\right\}+O(1)
\end{aligned}
$$

give the asymptotics of Theorem 1.1. The estimation $p_{\mu, \nu}, q_{\mu, \nu} \leq d_{\nu}-2$ follows from

LEMMA 7.11. For each $x_{0} \in\left[0, \widetilde{\xi}_{\nu}\right)$ there is some $2 \leq k \leq d_{\nu}$ with $\widetilde{y}_{\nu}^{(k)}\left(x_{0}\right) \neq 0$. If $j_{0}^{(\nu)}=1$ then the statement is valid also for $x_{0}=\widetilde{\xi}_{\nu}$.

Proof. Assume $\widetilde{y}^{(k)}\left(x_{0}\right)=0$ for $2 \leq k \leq d_{\nu}$. Then Taylor's theorem implies

$$
\widetilde{y}_{\nu}(x)=\widetilde{y}_{\nu}\left(x_{0}\right)+\widetilde{y}_{\nu}^{\prime}\left(x_{0}\right)\left(x-x_{0}\right)+O\left(\left|x-x_{0}\right|^{d_{\nu}+1}\right)
$$


in some neighbourhood of $x_{0}$ in $\left[0, \widetilde{\xi}_{\nu}\right]$. Consequently,

$$
1=\sum_{j=0}^{d_{\nu}} a_{d_{\nu}-j, j}^{(\nu)} x^{d_{\nu}-j}\left(\widetilde{y}_{\nu}\left(x_{0}\right)+\widetilde{y}_{\nu}^{\prime}\left(x_{0}\right)\left(x-x_{0}\right)+O\left(\left|x-x_{0}\right|^{d_{\nu}+1}\right)\right)^{j}
$$

and

$$
1-\sum_{j=0}^{d_{\nu}} a_{d_{\nu}-j, j}^{(\nu)} x^{d_{\nu}-j}\left(\widetilde{y}_{\nu}\left(x_{0}\right)+\widetilde{y}_{\nu}^{\prime}\left(x_{0}\right)\left(x-x_{0}\right)\right)^{j}=O\left(\left|x-x_{0}\right|^{d_{\nu}+1}\right)
$$

for $x$ close to $x_{0}$. The left hand polynomial of order $\leq d_{\nu}$ vanishes therefore at $x_{0}$ with order $\geq d_{\nu}+1$ and is consequently the zero polynomial. Therefore $\widetilde{g}_{\nu}\left(x, \widetilde{y}_{\nu}\left(x_{0}\right)+\widetilde{y}_{\nu}^{\prime}\left(x_{0}\right)\left(x-x_{0}\right)\right)=1$ on $\mathbb{R}$, which contradicts Lemma 4.1 .

\section{References}

[1] L. V. Ahlfors, Complex Analysis, McGraw-Hill, 1985.

[2] M. N. Huxley, Area, Lattice Points and Exponential Sums, Oxford Univ. Press, 1996.

[3] E. Krätzel, Lattice Points, Kluwer, 1988.

[4] G. Kuba and W. G. Nowak, On representations of positive integers as a sum of two polynomials, Arch. Math. (Basel) 58 (1992), 147-156.

[5] B. Lichtin, The asymptotics of a lattice point problem associated to a finite number of polynomials I, II, Duke Math. J. 63 (1991), 139-192; 77 (1995), 699-751.

[6] W. Müller and W. G. Nowak, On lattice points in planar domains, Math. J. Okayama Univ. 27 (1985), 173-184.

[7] —, - Lattice points in planar domains: Applications of Huxley's "Discrete HardyLittlewood Method", in: Number Theoretic Analysis, E. Hlawka and R. F. Tichy (eds.), Seminar Vienna 1988-89, Lecture Notes in Math. 1452, Springer, 1990, 139164.

[8] W. G. Nowak, Zur Gitterpunktlehre der euklidischen Ebene, Indag. Math. 46 (1984), 209-223.

[9] M. Peter, Gitterpunkte unter algebraischen Kurven, Habilitationsschrift, Freiburg, 1996.

[10] B. L. van der Waerden, Algebra I, 7. Auflage, Springer, 1966.

Mathematisches Institut

Albert-Ludwigs-Universität

Eckerstr. 1

79104 Freiburg, Germany

E-mail: peter@arcade.mathematik.uni-freiburg.de

Received on 24.4.1998

and in revised form 31.5.1999 